\title{
DOE/ID/13395--T/ \\ Enhanced Research in Ground-Penetrating \\ Radar and Multisensor Fusion with Application to the \\ Detection and Visualization of Buried Waste
}

Final Report on GRant DE-FC07-95ID13395

May 14, 1996

Submitted to:

GEORGE J. SCHNEIDER

U.S. DEPARTMENT OF ENERGY

IDAHO OPERATIONS OFFICE

850 ENERGY DRIVE

IDAHO FALLS, ID 83401-1563

Scientific Investigators:

ANTHONY J. DEVANEY

ChaRles DiMarzio

MITCH KOKAR

ERIC L. MILLER

CAREY M. RAPPAPORT

WILLIAM H. WEEDON

NORTHEASTERN UNIVERSITY

CENTER FOR ELECTROMAGNETICS RESEARCH

360 Huntington Ave., Boston, MA 02115

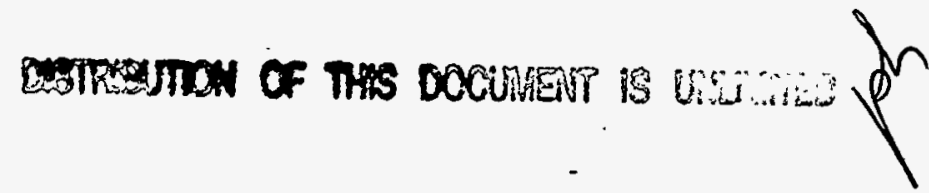

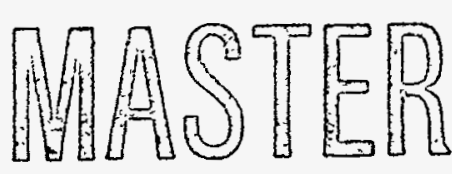




\section{Contents}

Forward.

William H. Weedon and Charles A. Dimarzio

CHAPTER I: Fusion of EM-61 and GPR Data for Pit Detection StePHEN P. LINDER AND MIECZYSLAW M. KoKaR

CHAPTER IIa: Inverse Scattering, Imaging and Object Detection Algorithms for Shallow Geophysical site Characterization

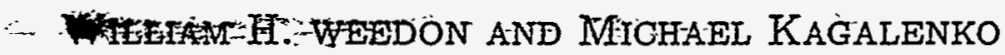

CHAPTER IIb: Tomographic Inversion of Time Domain Multi-Monostatic GPR Data

Ross W. Deming and Anthony J. Devaney

CHAPTER III: Object-Based Localization of Buried Waste Containers Using High Resolution Array Processing Techniques

Eric L. Miller and AdNAN Sahin

CHAPTER IVa: Laboratory Soil Characterization

WILLIAM H. WEEDON AND WEI SHI

Chapter IVb: Dispersive Soil Propagation Modeling

Wildiam H. Weedon, Carey M. Rappaport and Daniet SILEVITCH

Chapter V: Applications of Infrared Sensors to the Task of Landfill Remediation

Charles A. Dimarzio, Scott C. Lindberg and Michael J. ROSSACCI

Chapter VI:- Lidar for Detection and Monitoring of Fugitive Dust Emissions

Charles A. Dimarzio and Scott C. Lindberg 


\section{DISCLAIMER}

This report was prepared as an account of work sponsored by an agency of the United States Government. Neither the United States Government nor any agency thereof, nor any of their employees, make any warranty, express or implied, or assumes any legal liability or responsibility for the accuracy, completeness, or usefulness of any information, apparatus, product, or process disclosed, or represents that its use would not infringe privately owned rights. Reference herein to any specific commercial product, process, or service by . trade name, trademark, manufacturer, or otherwise does not necessarily constitute or imply its endorsement, recommendation, or favoring by the United States Government or any agency thereof. The views and opinions of authors expressed herein do not necessarily state or reflect those of the United States Government or any agency thereof. 


\section{DISCLAIMER}

Portions of this document may be illegible in electronic image products. Images are produced from the best available original document. 


\section{FORWARD}

This final report represents our research results on grant DE-FC07-95ID13395 from the U. S. Department of Energy, Idaho Field Operations Office for an initial 6-month research effort from October, 1995 through April, 1996. The grant, entitled Enhanced Research in Ground-Penetrating Radar and Multisensor Fusion with Application to the Detection and Visualization of Buried Waste has involved 6 Ph.D. level researchers, 7 graduate students, and 2 undergraduate students on 6 separate tasks (each task is a chapter in this final report). Recognizing the difficulty and importance of the landfill remediation problems faced by DOE, and the fact that no one sensor alone can provide complete environmental site characterization, a multidisciplinary team approach was chosen for this project.

Our initial results in this first six-month effort are very encouraging. Yet there is still much work to be done. We have developed a multisensor fusion approach that is suitable for the wide variety of sensors available to DOE, that allows separate detection algorithms to be developed and custom-tailored to each sensor. This approach is currently being applied to the Geonics EM-61 and Coleman step-frequency radar data. The processed radar and EM-61 images, along with associated detection algorithms, were not available until late in this project, so the results are still being verified.

A significant improvement was obtained in the quality of the results from the Coleman step-frequency radar. Calibration measurement data collected at the Coleman test-range in Orlando, Florida were significantly improved by removing through software discontinuities in the data due to a hardware phase instability. By applying a backpropagation algorithm, we found that we could essentially focus step-frequency radar data from a pipe in air down to a point. Improvements were also made in the step-frequency radar data collected from the glass-hole area at Brookhaven National Lab. There, backpropagated vertical crosssection radar images were integrated into surface profiles at various depth layers. The variable-depth surface profiles are particularly useful for finding pit locations and depth to buried waste, and show excellent agreement with the EM-61 and EM-31 images.

Pseudo-inverse scattering algorithms were also developed based on the Born approximation and and a farfield approximation for the incident and scattered fields. This algorithm was applied to experimental data, including the Sensors and Software impulse radar data collected at the Idaho National Lab. cold test pit.

High-resolution array processing techniques were developed for detecting and localizing buried waste containers. The MUSIC algorithm was modified to explicitly account for near-field physics, and provide direction of arrival estimates and consequently located the target using triangulation. The results obtained using simulated data are particularly encouraging.

A soil characterization laboratory facility was developed using a HP-8510 network analyzer and near-field coaxial probe. Both internal and external calibration procedures were developed for de-embedding the frequency-dependent soil electrical parameters from the measurements. We found that the external calibration procedure based on a quasi-static approximation that is typically used for this purpose is insufficient for frequencies above $500 \mathrm{MHz}$ and high-dielectric constant media. A new external calibration procedure is being developed. The goal is to eventually have a ruggedized soil characterization facility that can be taken to the field to perform on-site characterization for a particular environment. 
Dispersive soil propagation modeling algorithms were also developed for simulating wave propagation in dispersive soil media. These algorithms will utilize the soil parameters measured using the laboratory soil characterization techniques. These algorithms are extremely useful for predicting the performance of GPR systems for detecting targets in a given inhomogeneous dispersive environment. They are also useful for generating forward data for testing inverse scattering and data fusion algorithms.

A study was performed on the application of infrared sensors to the landfill remediation problem, particularly for providing information on volatile organic compounds (VOC's) in the atmosphere. However, this study was limited due to the lack of availability of IR data. Models were developed for characterizing IR system performance, measurement scenarios were considered, and the idea of using an IR camera in conjunction with a GPR system were examined.

A dust-emission lidar system is proposed for landfill remediation monitoring. Design a forications are-outlined for a system which could be used to monior dust emissions in a fandfill remediation effort.

The detailed results of our investigations are contained herein. 


\title{
Chapter I
}

\section{Fusion of EM-61 and GPR Data for Pit Detection}

\author{
STEPHEN P. LINDER ${ }^{1}$ AND MIECZYSLAW M. KOKAR ${ }^{2}$ \\ IDEPARTMENT OF MECHANICAL, INDUSTRIAL AND \\ MANUFACTURING ENGINEERING \\ ${ }^{2}$ Department of EleCtRICAL AND COMPUTER ENGINEERING \\ Northeastern UnIVERsity, Boșton, MA 02115
}

\author{
Final Report on Grant DE-FC07-95ID13395 \\ May 14, 1996
}




\title{
Fusion of the EM-61 and GPR Data for Pit Detection Progress Report, May 1996
}

\author{
Stephen P. Linder \\ Department of Mechanical, Industrial and Manufacturing Engineering
}

\author{
Mieczyslaw M. Kokar \\ Bepartment of Electrical and Computer Engineering \\ Northeastern University \\ 360 Huntington Avenue \\ Boston, Massachusetts 02115
}

\section{Introduction}

The goal of this sensor data fusion effort is to use complementary and/or redundant information contained in the data provided by different sensors to obtain better identification of objects such as waste pits, the water table, rocks, and to be able to differentiate these objects from clutter. In other words, the goal is to improve the ratio of probability of detection to probability of false alarm, the ratio of probability of correct classification to probability of misclassification and the accuracy of characterization (location, size, shape) of pits. One of the requirements for this effort is to develop techniques for data interpretation that provide, in addition to the characterization information mentioned above, some information regarding the certainty (or uncertainty) of the characterization. It is desirable that the characterization is represented in a visual form that is relatively easy to interpret by humans.

In this phase of the project we focused our efforts on three tasks: (1) development of fusion architecture, (2) development of fusion algorithms, and (3) initial testing of the developed algorithms on real data provided to us by the Idaho National Environmental Laboratory (INEL). For our experiments, we selected the EM-61 data and the GPR data from the Glass Hole site.

In this report we describe our fusion approach, our fuzzy logic based fusion algorithms and the results of our initial experiments with real data. The algorithms were also evaluated on simulated data; these tests served the purpose of checking the correctness of the fuzzy logic implementation. The simulation was limited to generating geometrical 
shapes (ellipses) of various sizes and at different locations. For each of the ellipses, the algorithm's fuzzy rules calculate the possibility of each detection ellipse being a waste pit. To verify the logic implemented in the rules we need to have the ground truth data. We have been provided with this kind of information in a graphical form. Wie translated the graphical representation into computer representation in which each pit was approximated by an ellipse. Visual evaluation of this implementation shows a close resemblance of the graphical representation. After testing our fuzzy rules on the simulated data, we applied these rules to the (provided to us) detection data from the EM-61 and the GPR sensors.

\section{Fusion Architecture}

The process of multisensor data fusion consists of several distinct steps:

- Data association: alignment of the data such that the measurements from different sensors may be represented in a common information space.

- Data abstraction: extraction of high-level features from raw or processed data.

- Incorporation of symbolic knowledge: process of combining background knowledge such as the types of objects we are looking for, features associated with objects and relations among features and objects, with features extracted from sensor data.

These processing modules can be incorporated into two main kinds of architectures: (1) fuse-then-abstract architecture and (2) abstract-then-fuse architecture. We have concluded that this first approach is not practical for this application due to the large number of different kinds of sensors, and the fact that these sensors report different types of information, and often in different physical spaces. For example, the data from a side-looking radar (SLUR configuration), after preprocessing and backpropagation is in the form of an image, where the horizontal axis represents distance along a surface scan line, and the vertical axis represents position along a 45-degree angle into the ground. The EM-61 sensor data used in our experiments, after gridding interpolation, is a surface image with the intensity representing the induced magnetic field at a point. The abstract-then-fuse approach is more appropriate for this kind of data. It also allows separate detection algorithms to be developed for each sensor, and be included in the overall fusion strategy: The basic structure of the data fusion processing system is presented in Figure 1.

In this figure, the Feature Extraction block implements detection; the output of this block was provided to us in the form of a list of detections. Each detection was characterized by $(x, y)$ coordinates of the center of detection, and $\sigma_{x}, \sigma_{y}, \sigma_{x y}$, parameters characterizing the sizes of the detected areas.

The Object Detection block implements fusion with the fuzzy reasoning about the possibility of particular ellipses being pits. This block is described in more detail in 


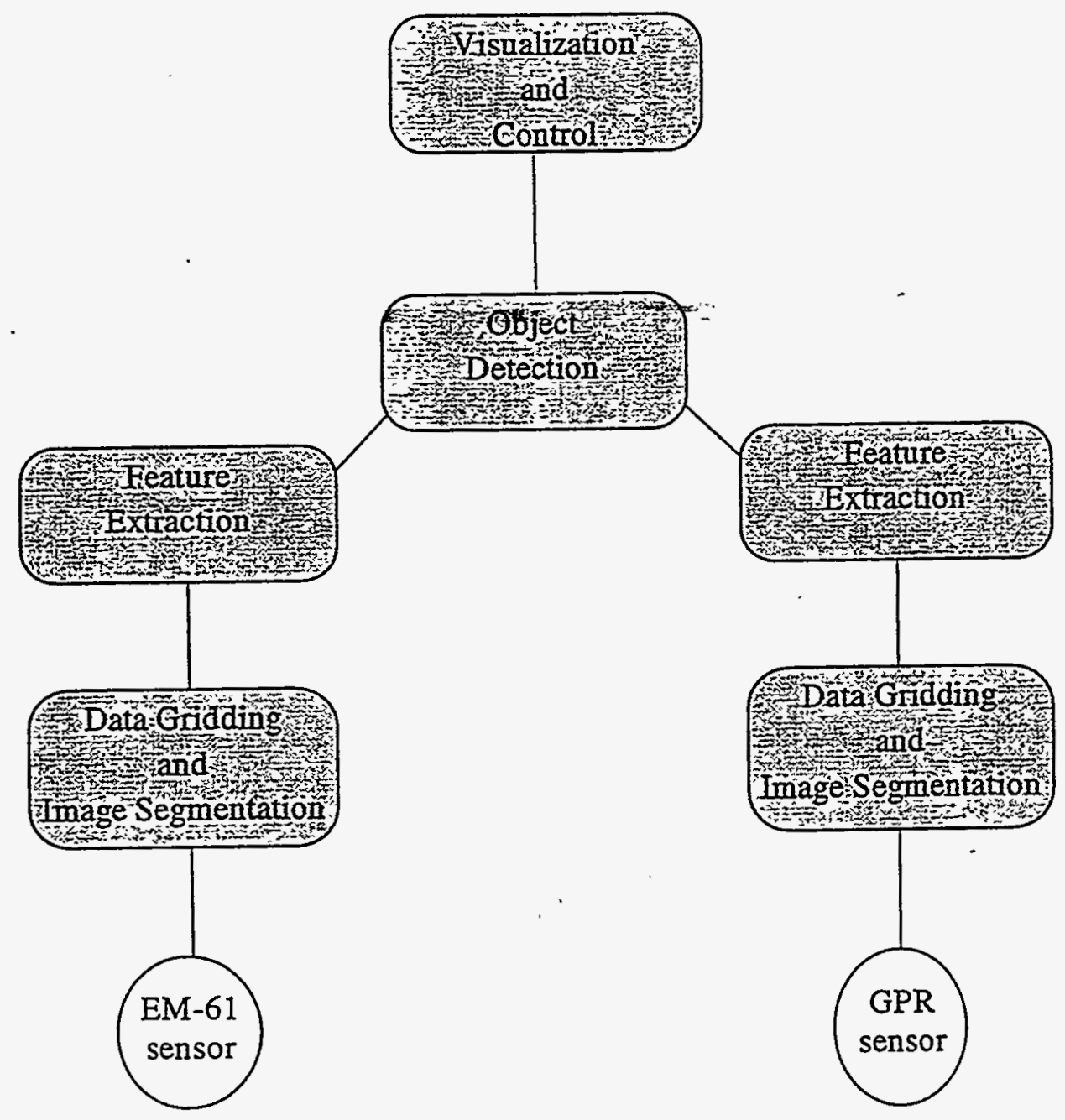

Figure 1: General Architecture of the Sensor Fusion System 
the next section. This module combines information extracted from raw data by the modules located below it in Figure 1 with background knowledge about both the domain of interest (waste buried in pits, waterbeds, clutter) and about particular sensors. The knowledge is both qualitative and quantitative. It deals with qualitative features such as ellipse, cluster, water-table, clutter as well as quantitative characteristics associated with these features, like $x, y, \sigma_{x}, \sigma_{y}, \sigma_{x y}$. Additionally, the knowledge must include information on the credibility of particular components of knowledge in order to give the means for assessing the certainty/uncertainty of particular conclusions.

\section{$3 \quad$ Fuzzy Logic Based Object Detection}

The symbolic character of the knowledge requires a logical framework for its representation and processing with a mechanism for incorporating uncertainty. One approach that we are currently investigating is fuzzy logic. One of the advantages of fuzzy logic techniques is the ability to represent qualitative knowledge in a language that is relatively easily translatable from the natural language. An example of such a fuzzy element of knowledge (rule) is the rule for reasoning about location of a pit based on reports from two sensors. Fusing position measurements from two sensors could be achieved simply by taking the average of the two positions. However we can create a theory for fusing these measurements that exploits the physical knowledge we have of the sensors. We have developed an initial theory of how to fuse position measurements from GPR and EM-61 and implemented a system using fuzzy logic.

The system contains rules that evaluate the relative merit of each measurement based on the size of the detected object (calculated from its $\dot{\sigma}_{x}$ and $\sigma_{y}$ ), total weighted size of its GPR neighbors, and total weighted size of its EM-61 neighbors. This algorithm presumes that the image has been segmented prior to its application. We discuss segmentation in the next section.

According to the fuzzy logic theory, each of the fuzzy (linguistic) variables, in this case these are size of an ellipse, mean distance to its neighbors and total size of its neighbors, has values in a fuzzy set. A fuzzy set $A$ differs from a set in the traditional meaning, in that its characteristic function $\mu_{A}$ takes on values from an interval $[0,1]$ rather than from the set $\{0,1\}$. This function is said to determine the degree of membership. An example of such functions for our application is shown in Figure 2. We selected four linguistic values for our variables: tiny, small, medium and large. Note that any quantitative value can be classified in more than one fuzzy set, but its degree of membership may be different for each set. For instance, the size of an ellipse may be small with the degree of 0.8 and medium with the degree of 0.25 . Note also, that these degrees of membership cannot be interpreted as probabilities. For instance, if a person is four feet tall, the degree of membership in the set short can be 0.99 . Certainly, the probability of that person of being short is not 0.99 ; this simply depends on the population of people we are dealing with. Fuzzy logic is easy to interpret in terms of multivalent logics in which the truth value of a proposition is not simply 0 or 1 , but can be anywhere in between. In fuzzy 
logic, the logical connectives, like $\wedge$ and $\vee$, are interpreted as operations on fuzzy sets. Typically, the value of a conjunction is computed using the min function, and the value of a disjunction is computed using the max function.

In our system we have implemented a number of rules for reasoning about pits (represented by ellipses). An example of a fuzzy rule is:

If (Sensor Type is gpr) and (Target Size is medium)

and (Weighted Size of GPR Neighbors is small)

and (Weighted Size of EM-61 Neighbors is medium)

then (Pit-Possibility is large) (1)

The weithted size of the neighborhood pit $n$ is calculated using the following equation:

$$
\sum_{i \neq n} \frac{\operatorname{size}(i)}{\operatorname{dist}(i, n)}
$$

The system calculates the value of Pit-Possibility (we call it pittness) for each ellipse. Note that the value large carries the the entire possibility distribution, like the one shown in Figure 2. Also, for the same ellipse, multiple rules can generate different distributions. The final outcome then is computed using the interpretation of the conjunction operator described above. An example of the result of the application of such rules is shown in Figure 3. GPR detections that overlap EM=61 detections are reasoned to have a higher possibility of being a pit since our rules incorporate the domain knowledge that all pits have metal.

We have developed a number of rules that collectively constitute theories characterizing pits, waterbeds, clutter, and sensors. We have tested our approach on simple synthetic scenarios. Examples of such scenarios are:

1. Single pit, uniformly distributed clutter, objects detected by EM-61 are ellipses, GPR's objects are ellipses parallel to the surface (fixed depth).

2. Single pit, uniformly distributed clutter, objects detected by EM-61 are ellipses, . GPR's objects are ellipses, watertable objects detected by both EM-61 and GPR.

3. Pits found by EM-61 and GPR are of different sizes.

4. Some of the objects detected by one sensor but not by the other.

\section{Image Segmentation}

The fuzzy system described in the previous section had an underlying assumption that the input was a segment of an image, rather than the whole image. This assumption was fulfilled in the simulated data, since we simulated ellipses within a relatively small 


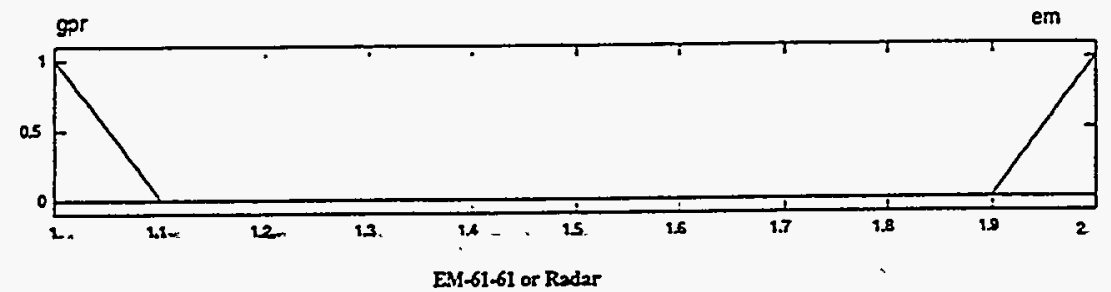

1
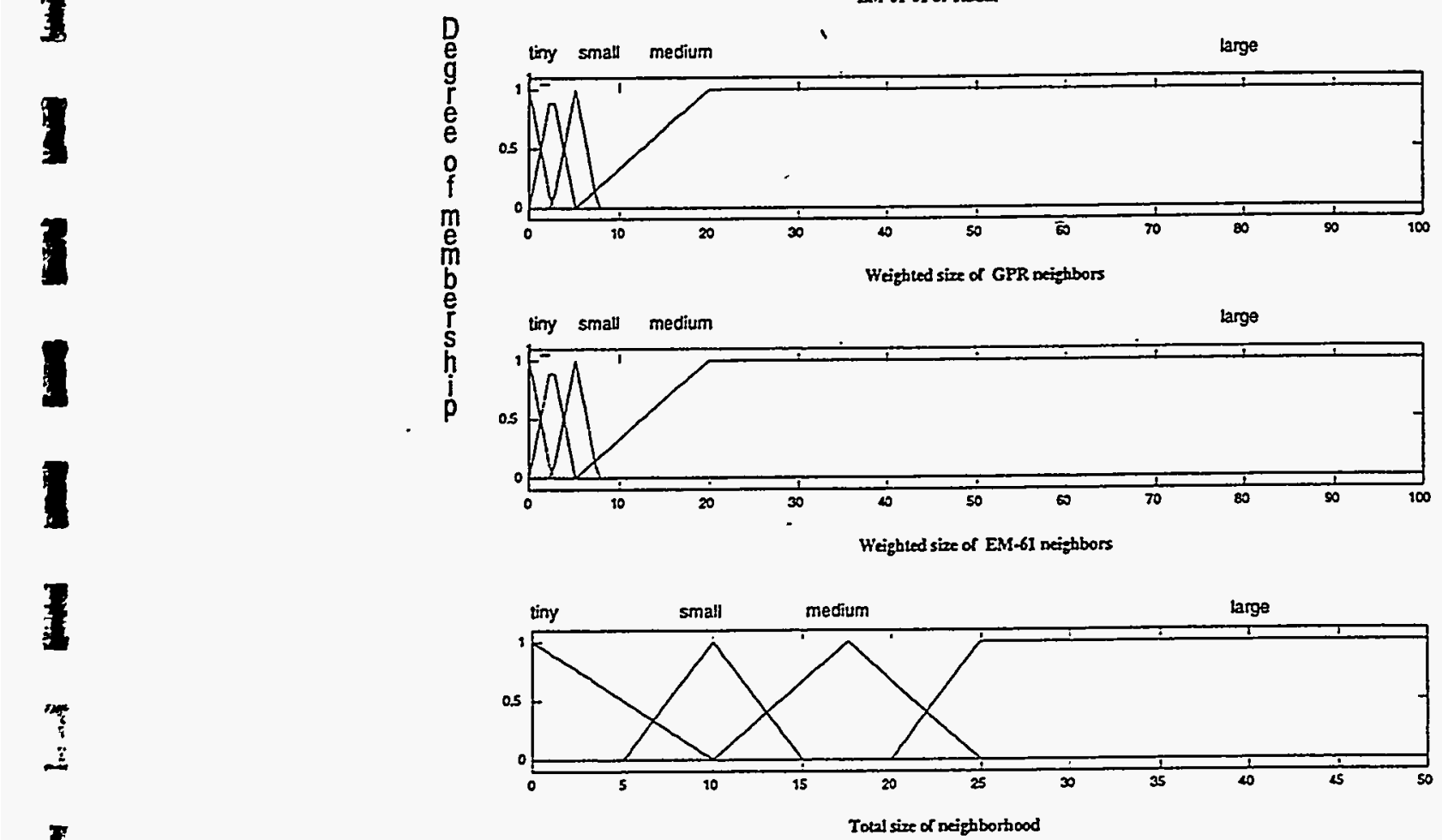

Figure 2: Membership Functions 


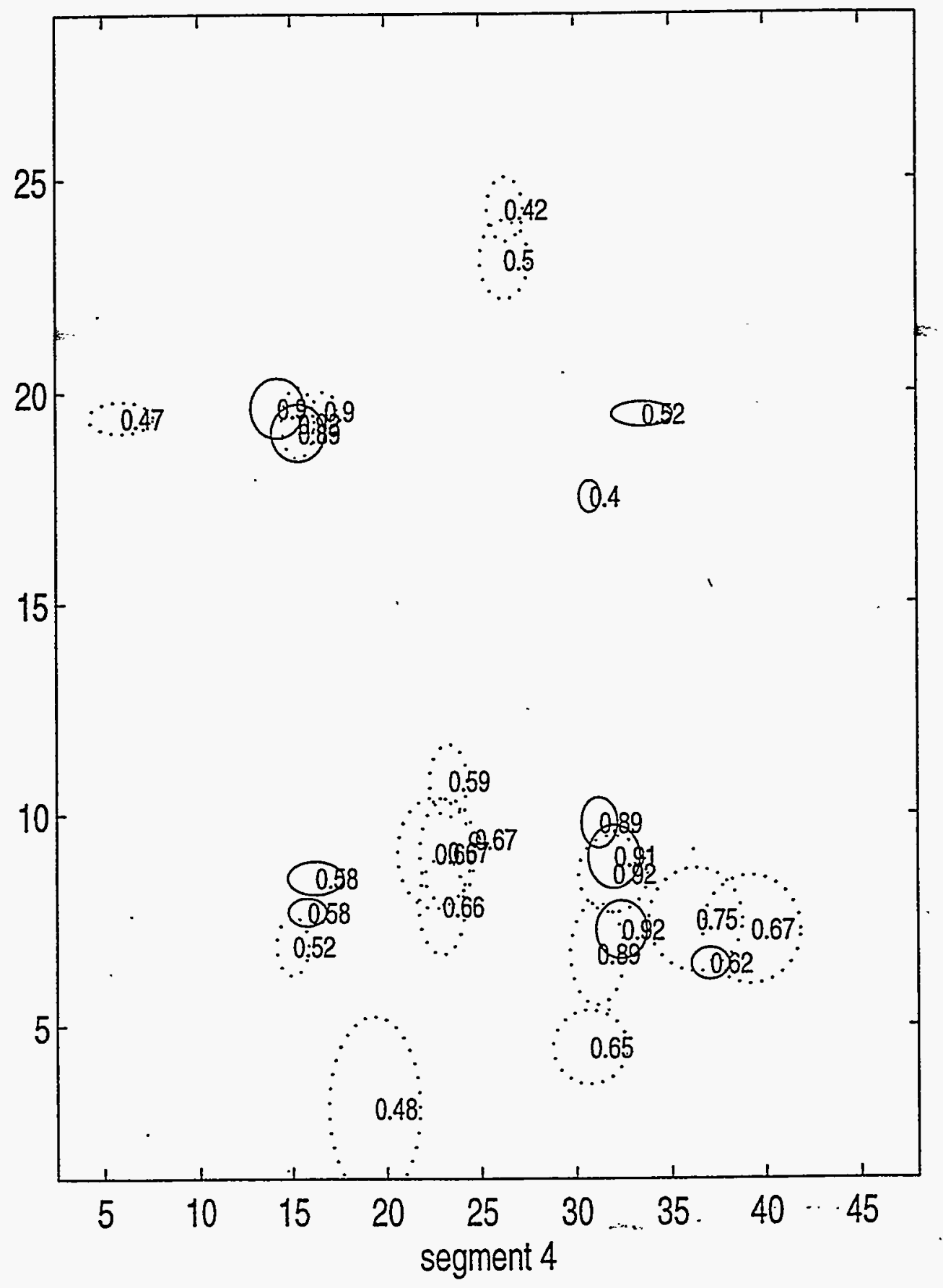

Figure 3: Values of "Pittness" 
neighborhood. For real data, however, detections are spread over the whole image. In order to run the algorithm described in the previous section, the image must be first segmented. In our experiments we used the fuzzy clustering algorithm whose basic structure is provided with the Fuzzy Toolbox of Matlab. An example of clustering using this algorithm is shown in Figure 4.

\section{$5 \quad$ Fused Object Detection and Identification}

In order to fuse the signals from two different sensors we first need to project the detections from the two sensors onto a common space. We used a sliced representation of the physical space for this purpose. In this model, we can interpret the EM-61 data as a projection on one $(x, y)$ plane. The GPR data can be interpreted as composed of a number of slices. This model is shown in Figure 5. In our initial experiments we used only one projection of the radar data. Both the EM-61 data and the projected radar data that we were provided with is shown in Figure 6.

In order to evaluate the correctness of the algorithms, we need to compare the result of the algorithm with the ground truth. We were given a report from Soft Earth Associates, which contains estimates of the ground truth (in the form of a map). We treat this data as ground truth. In order to be able to manipulate it by a computer, we digitized this map as a collection of ellipses, similarly like the detection data from the sensors.

Having this ground truth, we were able to compare the correlation of the detections from both the EM-61 sensor and from the GPR. The results of this comparison are shown in Figures 7 and 8. As we can see from these figures, the correlation between the detections from both sensors and the ground truth is rather low. This low correlation makes it difficult to assess the quality of the fusion algorithms. Before we can assess the quality of the fusion algorithm the question of whether the detections from the two sensors are not precise or the ground truth is not correct must answered first. The reason for questioning the truth data is that the detections from both EM-61 and GPR are more closely correlated to each other than either of them are to the ground truth.

We ran our fusion algorithm on the data shown in Figure 6. The result of this fusion is shown in Figure 9.

\section{Conclusions}

In this six-month period we have accomplished a number of tasks. We developed an architecture in which detection can be carried out separately for each sensor's data and fusion can be performed on these detection data sets. We have experimented with an image segmentation algorithm, which is based upon a fuzzy clustering algorithm. We have implemented a fuzzy system for reasoning about pits in each data set separately and in both data sets (fusion) concurrently. We have developed a method for comparing 


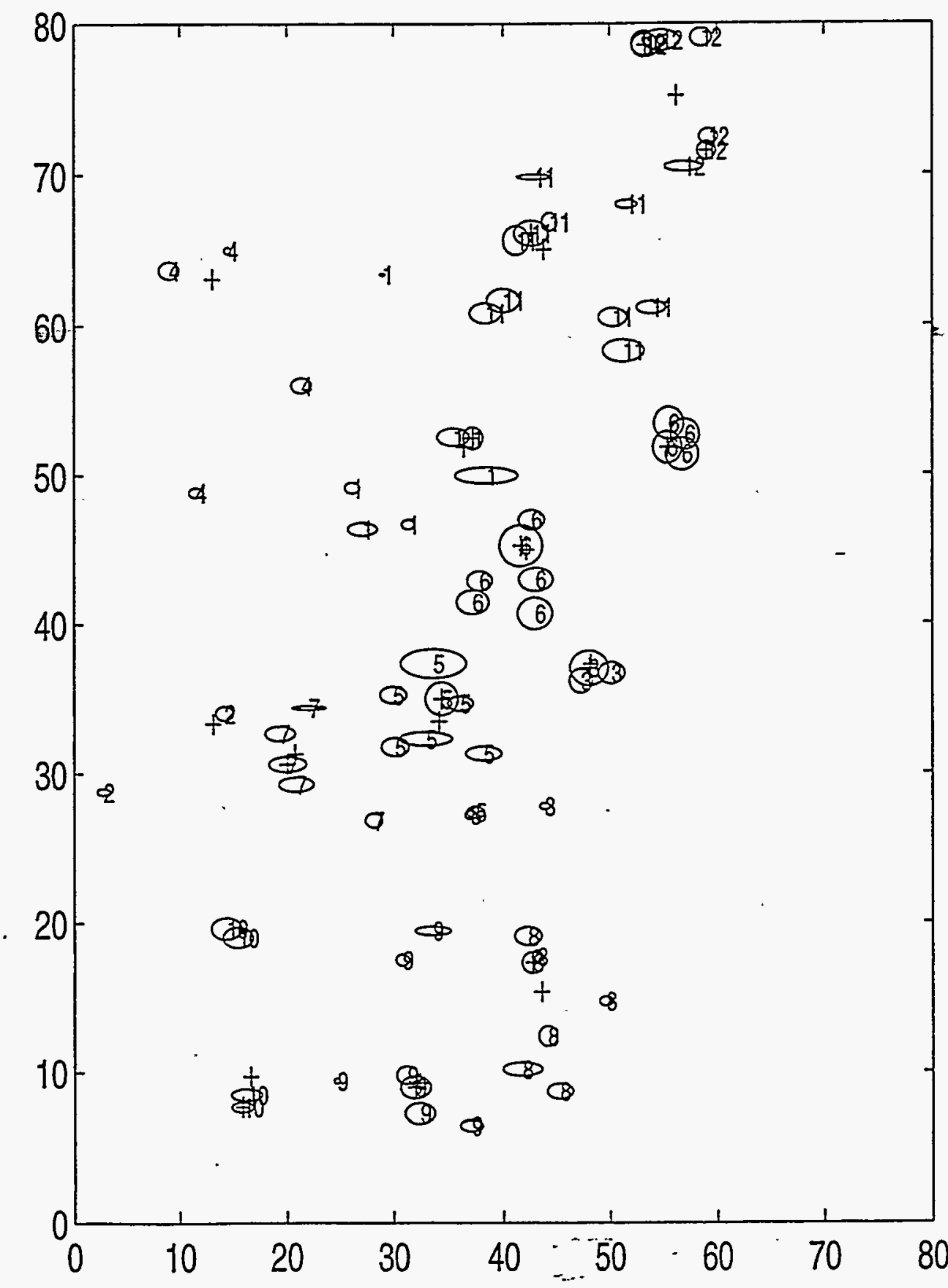

Figure 4: Result of Clustering 


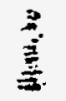

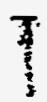

1

$\mathrm{x}$

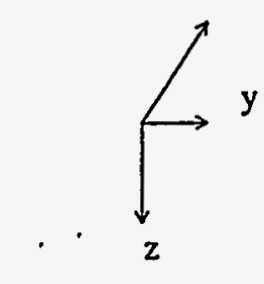

Figure 5: Projections of the EM-61 and GPR Data

1

1

1

1

]

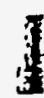

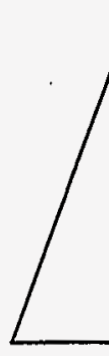

O

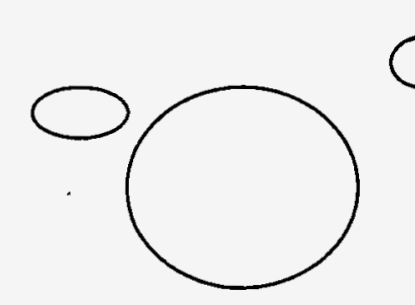

$\bigcirc$

EM-61 data
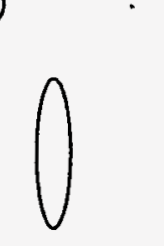

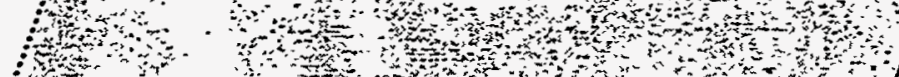

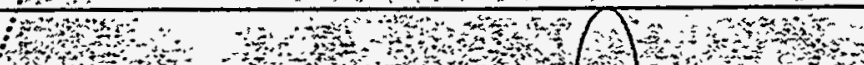
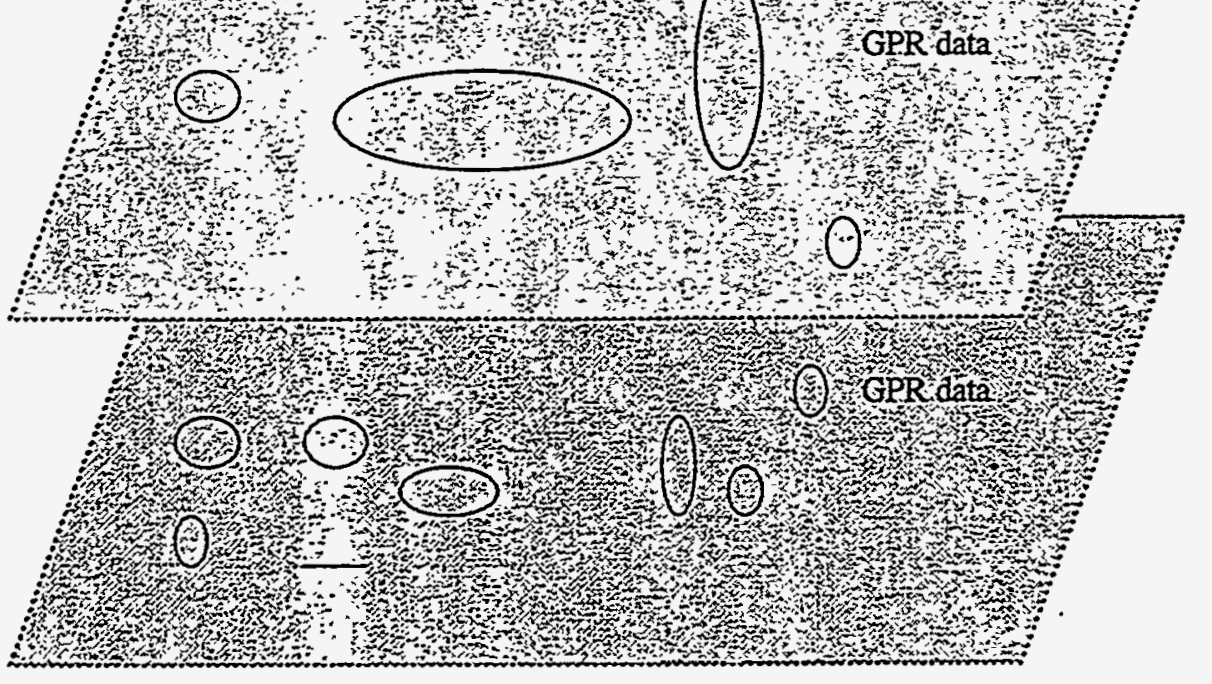

1

(1)

Figu 5 Projections of the EM-61 and GPR Data 


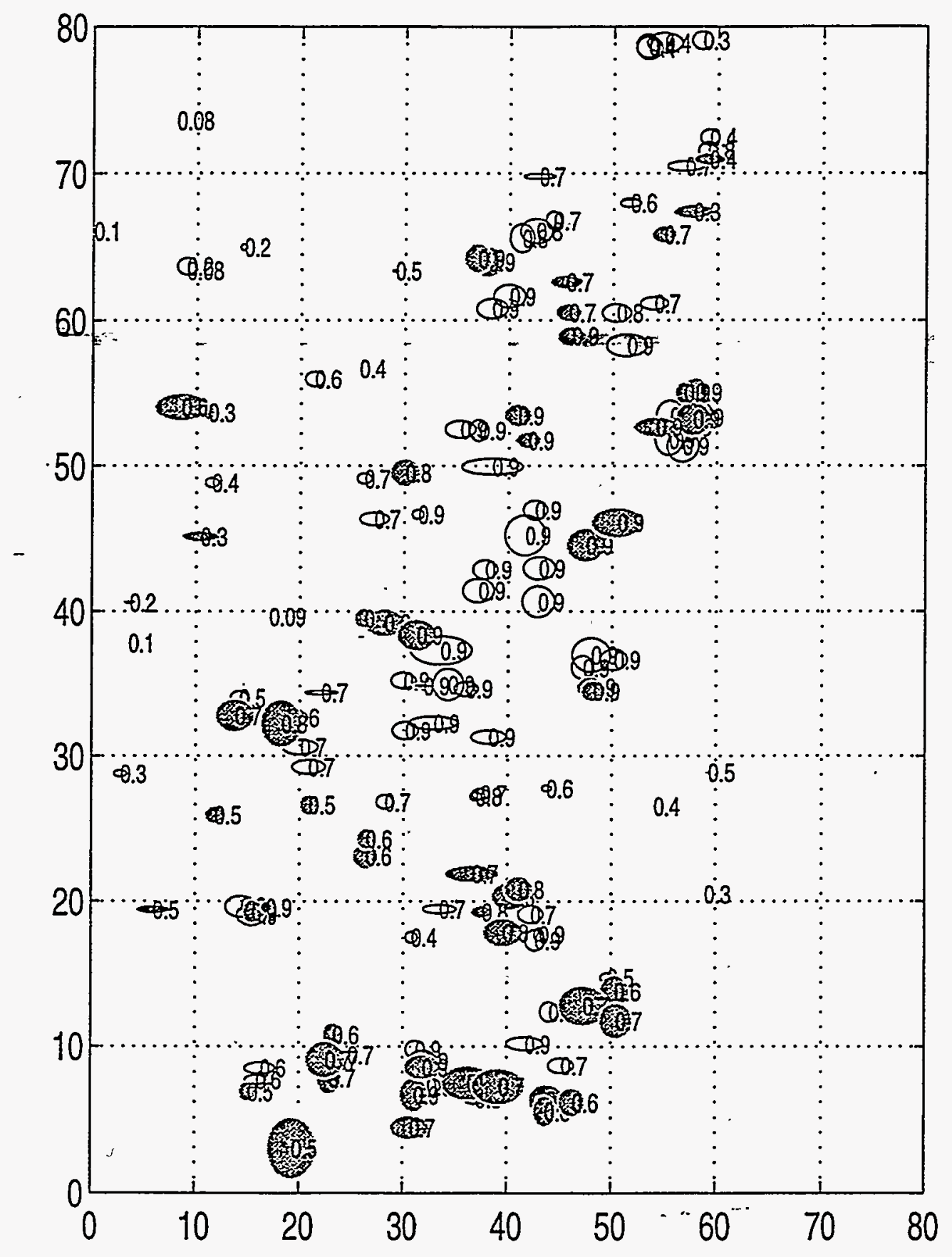

Figure 6: Projection of the GPR Data (gray) and EM-61 data 


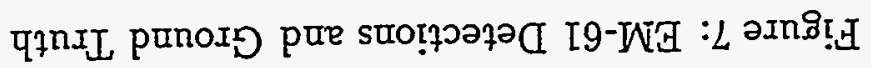

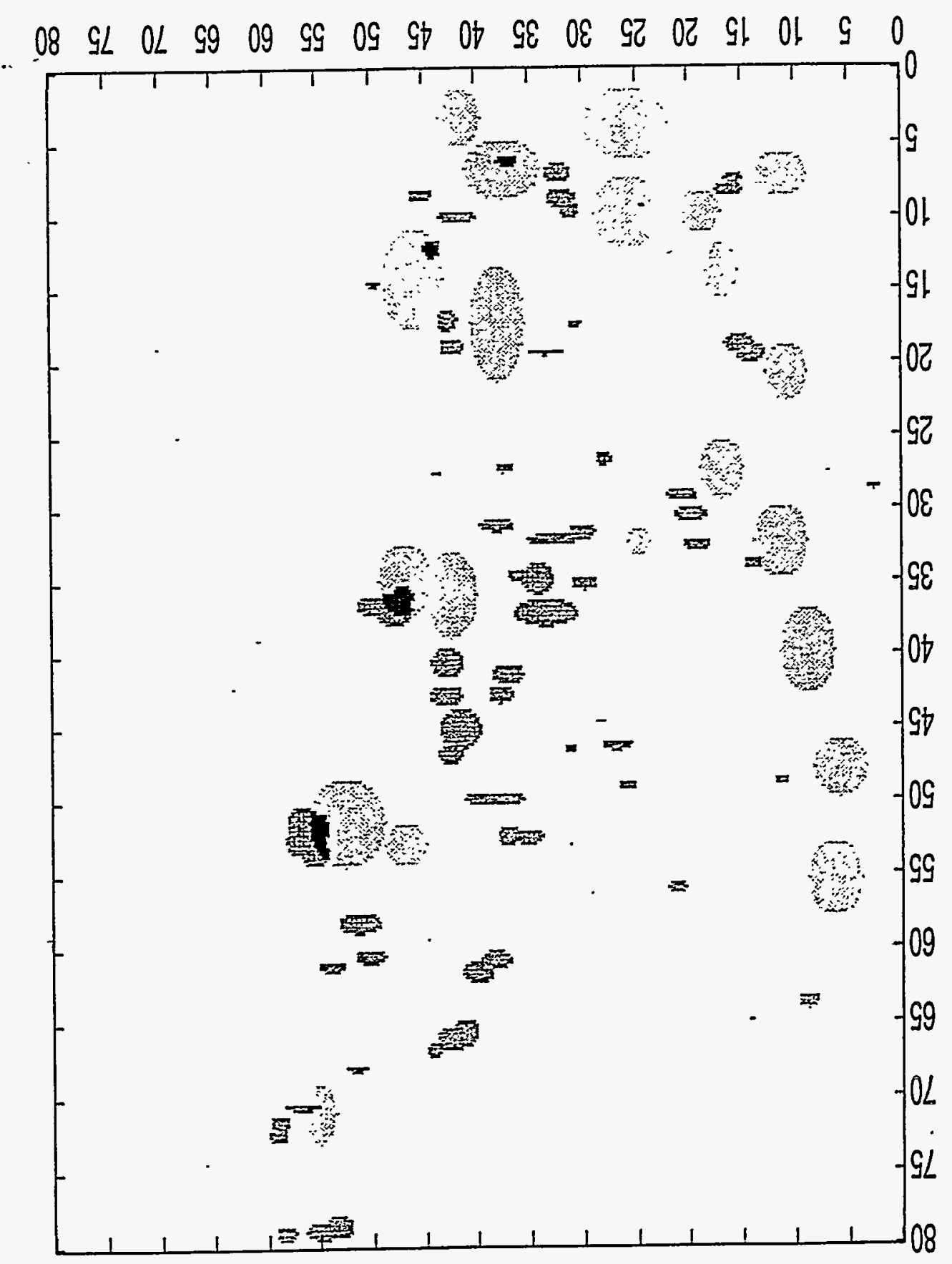

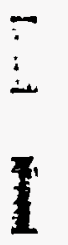

I

I

I

I

I

I

I

[

I

I

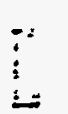

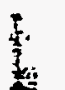




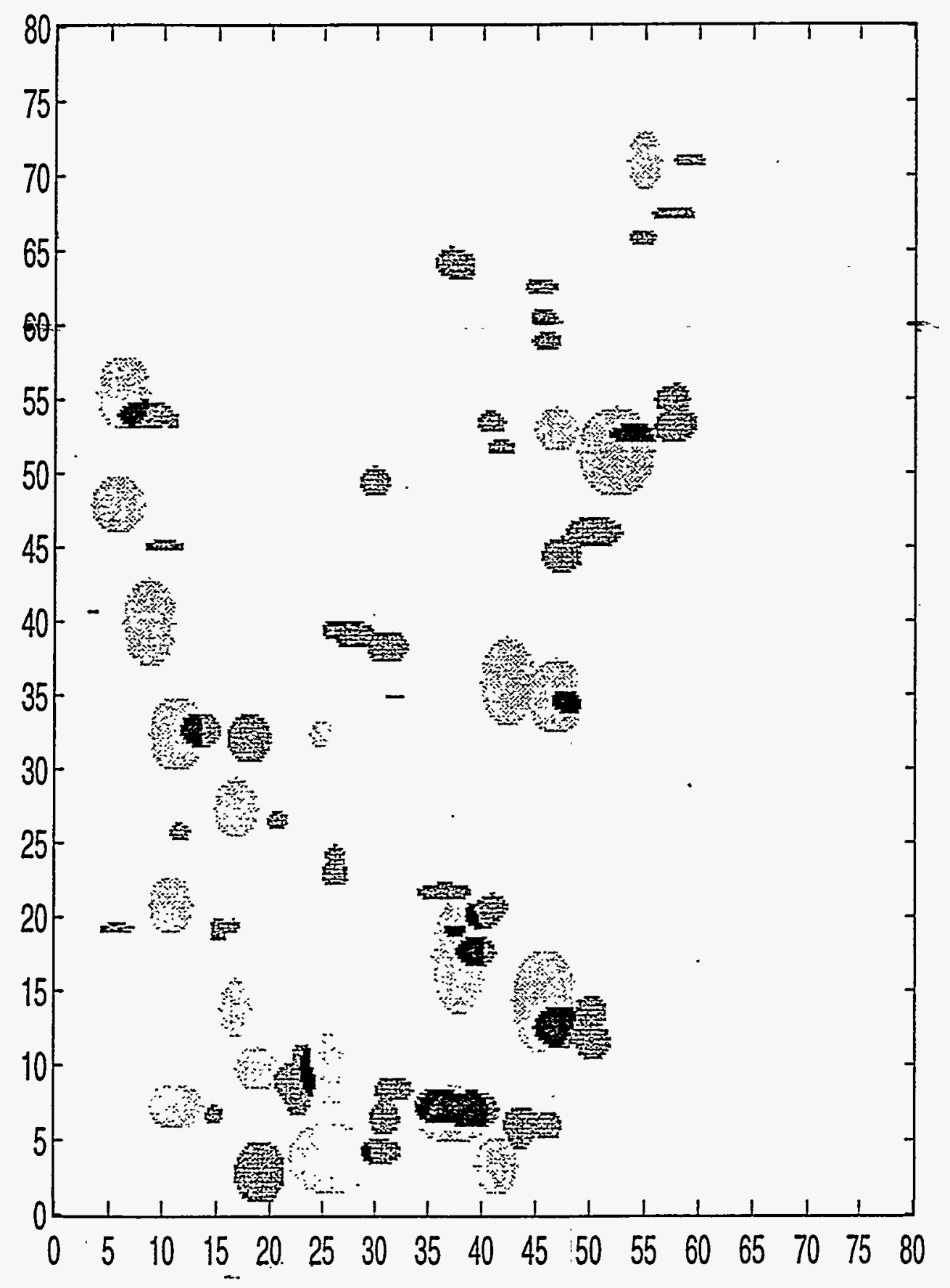

Figure 8: GPR Detections and Ground Truth 


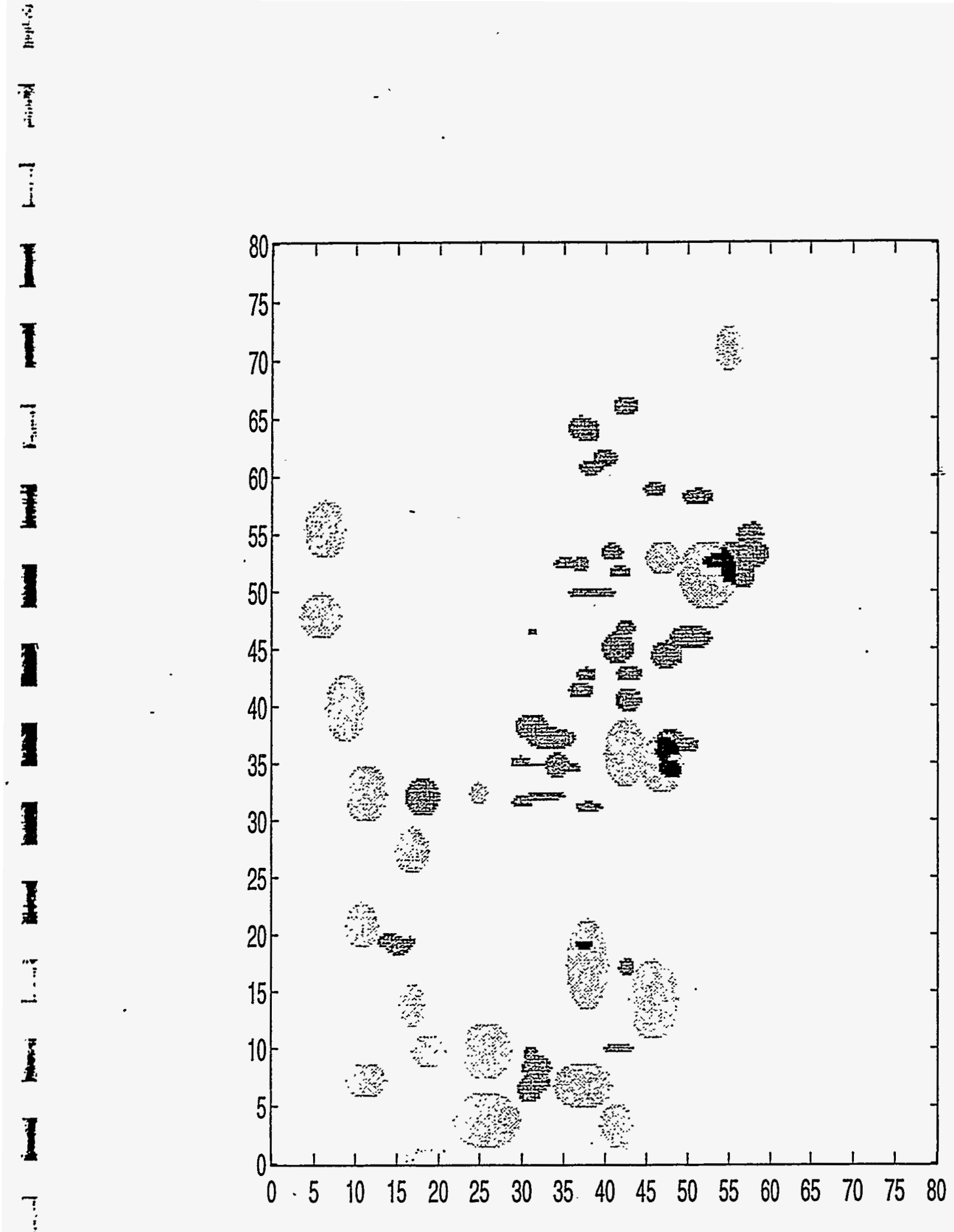

Figure 9: Fused Detections and Ground Truth 
the results of the algorithms with respect to the ground truth, which will be very useful in our future research. We have also performed initial experiments with the real detection data from the two data sets (EM-61 and GPR). Overall, in our first six-month effort we have been able to demonstrate that the proposed approach, as described above, can be applied to the task of detecting and characterizing pits, recognizing waterbeds and clutter.

\section{Future Research}

The experiments were carried out on simple synthetic scenarios. The main effort of the second six-month period should be focused on both expanding the knowledge about the domain and the sensors, as well as on fine-tuning of this knowledge. As mentioned earlier, the representation of knowledge includes information about uncertainty. The result of processing strongly depends on the assignment of uncertainty to particular elements of the knowledge. There are mechanisms for adaptive tuning of this kind of knowledge. These methods require iterative access to data. This requirement is relatively difficult to fulfill with real data. We propose that the real data be used only for the fine-tuning, while coarse tuning be performed based upon simulated data. The simulated data could be also used for learning (either automatic or human) of the patterns that can be incorporated in the knowledge base of the system.

The tasks to be addressed under the second six-month effort include the following:

1. Investigate the issue of the ground truth.

2. Develop scenarios of progressing complexity for both EM-61 and GPR to be used for testing of the fusion algorithms and for learning new rules.

3. Develop a simulation program for the EM-61 sensor that implements the developed scenarios.

4. Expand the existing simulation program for GPR to incorporate the developed scenarios.

5. Expand the knowledge base that captures qualitative/quantitative information about both the domain of pit detection/characterization and the sensors.

6. Investigate the possibility of pixel to pixel fusion of the EM-61 and the GPR data.

7. Develop prototype algorithms for visualizing results of processing at various levels.

8. Tune the knowledge base using the developed simulations.

9. Test the fusion system on the simulated data.

10. Perform initial tests on the real EM-61 and GPR data. 
11. Analyze results. Identify topics for future research.

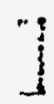

7

$+$

羑

1

1

1

I

1

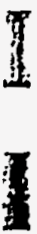

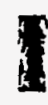

I

I

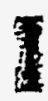

1 


\title{
Chapter IIa
}

\section{Inverse Scattering, Imaging and Object Detection Algorithms for Shallow Geophysical Site Characterization}

\author{
William H. WeEdon and MichaEl KagalenKo \\ DEPARTMENT OF ELECTRICAL AND COMPUTER ENGINEERING \\ Northeastern University, Boston, MA 02115
}

Final Report on Grant DE-FC07-95ID13395

May 14, 1996 


\title{
Inverse Scattering, Imaging and Object Detection Algorithms for Shallow Geophysical Site Characterization
}

\author{
William H. WEEDON AND Michael KagalenKo \\ CENTER FOR ELECTROMAGNETICS RESEARCH \\ DEPARTMENT OF ELECTRICAL AND COMPUTER ENGINEERING \\ NORTHEASTERN UNIVERSITY, BOSTON, MA 02115
}

\section{Introduction}

Interpretation of geophysical data such as ground-penetrating radar (GPR), low-frequency EM, magnetic, and conductivity data is greatly improved with accurate physical modeling of both the sensors and their interaction with the underground environment and scattering objects. The goal is to develop algorithms that can detect and identify underground targets of interest either by generating an image of a subsurface region or through correlation with signatures of known targets. The primary difficulties limiting performance are: 1) attenuation and waveform dispersion due to soil moisture; 2) clutter due to rock, soil layers, random metallic scatterers, and other inhomogeneities; 3) surface reflections which reduce the amount of energy coupled into the ground and cause "ringing" that make it difficult to locate shallow buried objects; 4) inaccurate or lack of physical models for the - sensors and environment.

The goal of this research is develop algorithms to process and interpret data collected from several geophysical sensors currently available to DOE. The processed data would then be passed to a fusion processor (see related sensor data fusion task) either as processed radar images or symbolically as a link list of feature detection parameters. For this work, we decided to focus our efforts on the glass-hole area at Brookhaven National Lab., due to the abundance of high-quality GPR, magnetic, and conductivity data, and the active interest in the site.

Three complementary data sets from the BNL site that we chose to include in our analysis are: 1) Coleman Research, Inc. step-frequency radar; 2) Geonics EM-61 metal detector; and 3) Geonics EM-31 conductivity mapping device. These data sets are complimentary in the sense that the EM-61 only detects metallic anomolies, while the EM-31 detects variations in soil conductivity. The radar is sensitive to metallic scatterers, conductivity variations, as well as dielectric scatterers, including rocks and soil layers. The EM-61 detects pits that contain metals, such as laboratory chemical jar caps, in addition to random scatterers. The EM-31 could detect pits that do not contain metals, but do have a significant conductivity variation from the background. The GPR is also good at detecting disturbed earth, where changes in soil density caused by digging and repacking a buried waste hole induce changes in soil permittivity that are detected by the GPR.

\section{Radar Imaging Algorithms}

Most commercial GPR systems in current use are impulse radars, that transmit an impulsive time-domain waveform with a pulse width on the order of 1 ns. Radar data is collecting by towing the radar and antenna assembly along the ground surface and collecting averaged time waveforms for several points along a scan line. The "raw" radar is often interpreted as a 2-D image with surface position along the horizontal axis and time in nanoseconds along the vertical axis. Appropriate time-variable gain is used to account for spreading and attenuation. A similar type of crude radar interpretation could be used with a step-frequency radar, similar to the one manufactured by Coleman, by applying a 
windowed temporal inverse FFT.

Quite often, this crude analysis of the raw radar data is enough to detect anomolies in the data and thereby locate buried objects. The raw radar data contains many scattering effects including diffaction, attenuation, multiple scattering that often makes interpretation of raw data difficult. Moreover, raw data interpretation does not allow for the incorporation and removal of nuisance effects due to the host medium, including velocity variations and soil layers, and known scatterers.

To go a step beyond the raw data analysis, we investigated and utilized two radar imaging algorithms. The first is a systhetic aperture imaging (SAI) algorithm currently used by Coleman to interpret their radar data. The second is a backpropagation imaging (BPI) algorithm, based on the angular spectrum expansion. We have compared the two algorithms using numerical experiments, and have found that the BPI is superior in terms of resolution and computational resources, and generates fewer "ghost" artifacts. A conference paper was submitted and accepted based on a this comparison [I].

\section{Coleman Step-Frequency Radar Interpretation}

We have put a considerable amount of effort into processing and interpreting the Coleman Research, Inc. step-frequency radar (SFR) data. Two separate sets of Coleman SFR data were investigated, and in both cases high-quality radar images were generated. The first set consisted of calibration measurements conducted at the Coleman test range in Orlando, FL [2]. The second set was actual field data collected at Brookhaven National Lab. in the glass-hole area during April, $1995[3,4]$.

A diagram of the Coleman SFR antenna sled is shown in Figure 1. The antenna consists of three broadband spiral antennas: one transmitter located in the center, and two receiving antennas. One receiving antenna is co-polarized with respect to the transmitter, while the other is cross-polarized. The step-frequency radar operates from $160 \mathrm{MHz}$ to $1058 \mathrm{MHz}$ in $2 \mathrm{MHz}$ steps. It is a bistatic system with a right-hand circularly polarized transmit antenna and both left-handed and right-handed circularly polarized receive antenns. The cross-polarized signal is stored as channel-1, while the co-polarized signal is stored as channel-2.

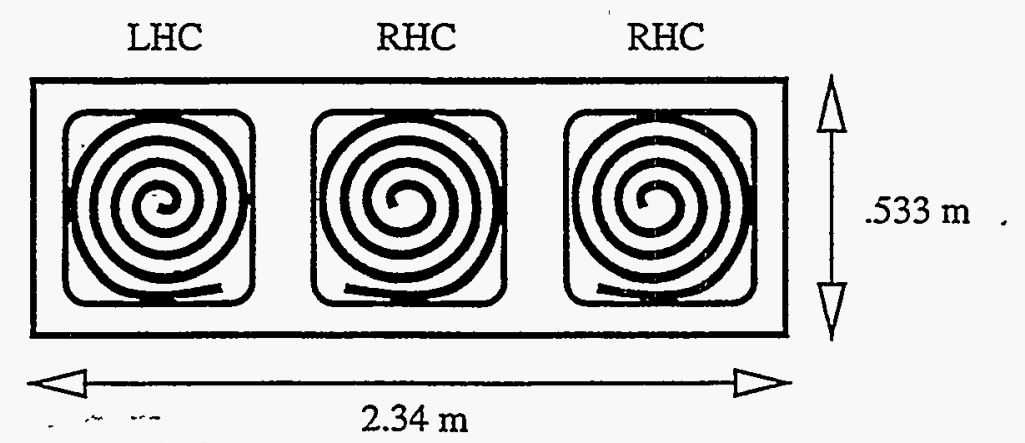

Figure 1: Diagram of the Coleman Research, Inc. step-frequency antenna sled.

\subsection{Calibration Measurements}

Before investigating actual field data collected by the SFR system, we found it useful to investigate some calibration measurements that were collected by Coleman at their factory test facility. The calibration radar images summarized in the calibration measurement 
technical report [2] were not of high quality, and were plagued with many unexplained features. When we investigated the actual radar data, we found that the data itself was actually good data. The unexplained features in the data turned out to be effects due a small phase instability, that could be removed with processing.

Figure 2 shows the results of our pre-processing to a calibration target consisting of a 1-inch diameter metallic pipe suspended in air. In our pre-processing, we performed a windowed inverse FFT, correlation-aligned the data, and subtracted the direct-air wave. The reflection from the pipe stands out nicely, and exhibits the typical "reflection hyperbola" shape. Figure 3 shows the results of our backpropagation algorithm applied to the data. Note that we can essentially focus the pipe reflection data down to a point.

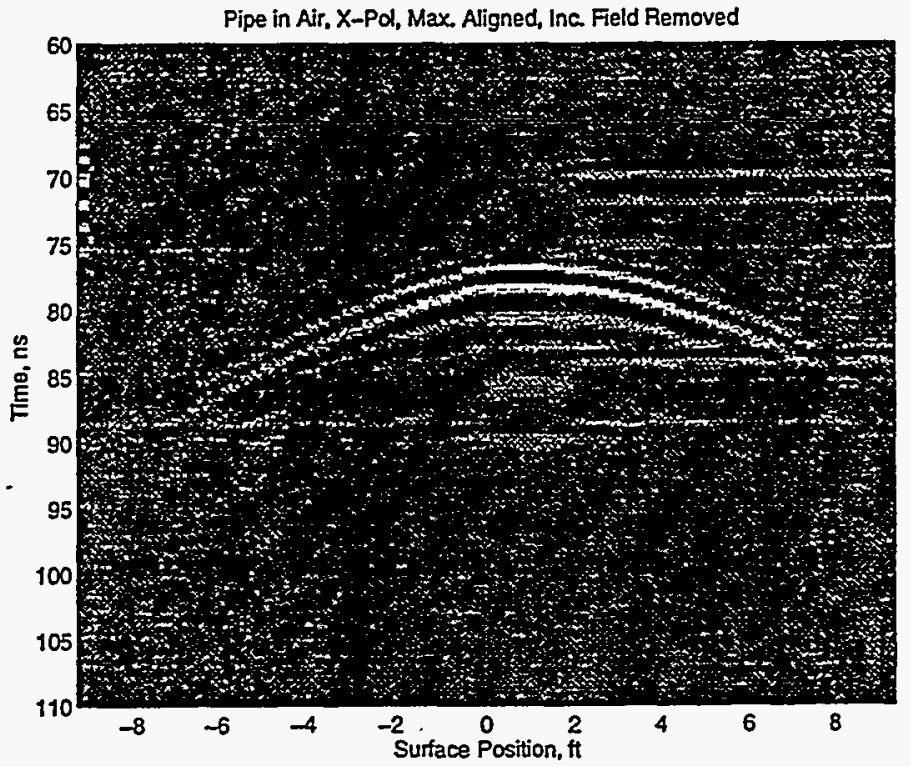

Figure 2: Pre-processed time-domain data from Coleman step-frequency radar. Calibration target consists of a 1-inch diameter metallic pipe suspended in air.

\subsection{Field Measurements: Brookhaven Glass-Hole Area}

Field measurements were collected with the Coleman SFR system at the glass-hole area of Brookhaven National Lab. in April, 1995. The glass-hole area is a 4800 sq. meter landfill used for disposal of rinsed laboratory glassware between 1966 and 1981, but now believed to contain intact chemical containers. There were a total of 140 scan lines collected by Coleman, containing both X-and Y-direction scan lines, each line averaging roughly 4 Mbytes of data, or 600 Mbytes total radar data.

Figure 4 shows a typical line of pre-processed data collected at the site. Note the significant amount of "ringing" in the first $25 \mathrm{~ns}$ of time. This problem is believed to be related to a mismatch of the antenna with the soil, and should probably be fixed in hardware. In fact, all GPRs exhibit a certain amount of surface ringing, but it is desirable to reduce this effect as much as possible with hardware. This early-time ringing is troublesome for detection and imaging of shallow ( $1-2$ meter depth) buried waste and other objects, such as unexploded ordinance, since the reflection signature from the real targets (waste) can easily be lost in the surface ringing. We are currently investigating 


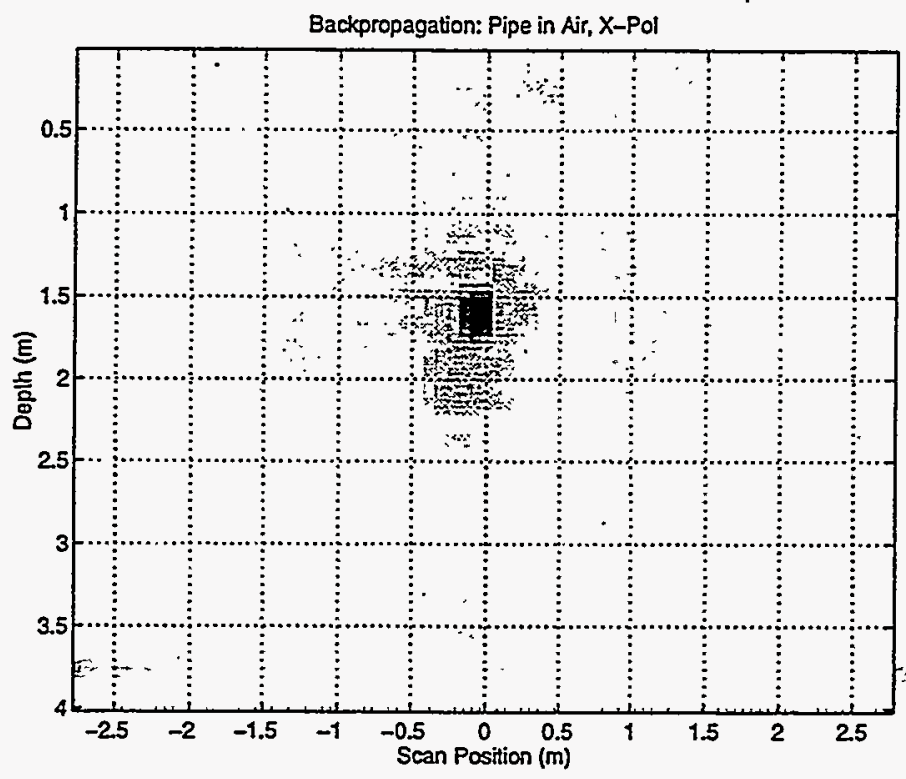

Figure 3: Backpropagation image of calibration target consisting of a metallic pipe in air. The backpropagation essentially focuses the pipe target down to a point.

several approaches for removal of the surface ringing with processing. The $25 \mathrm{~ns}$ round-trip time, assuming a dielectric constant of $\epsilon_{r}=6.3$, which we believe to be accurate for the site, corresponds to a depth of $1.5 \mathrm{~m}$. Hence, it would be difficult to detect objects in the first (1.5 meters) of soil without removal of the surface clutter.

Figure 5 shows a backpropagation of the data shown in Figure 4. To eliminate effects due to surface clutter, we have backpropagated the data from depth ( $z=1.5$ meters) to $(z=6.0$ meters $)$ only.

Location of pit boundaries from the raw radar data or backpropagated images is a tedious task, due to the large number of lines of radar data. To circumvent this difficulty, and make identifiction and location of the pits easier, constant depth-layer images were generated by integrating the backpropagated images for various depth-ranges. The result is a sequence of surface images, representing constant depth layers. Figures 6-10 show Channel-1 (cross-polarized) surface images for 1.5 - 2.0 meters, 2.0 - 2.5 meters, 2.5 - 3.0 meters, 3.0 - 3.5 meters and 3.5-4.0 meters. Similarly, Figures 11-12 show the channel-2 (co-polarized) images for 1.5 - 2.0 meters and 2.0 - 2.5 meters. The channel-1 (crosspolarized) images clearly identify strong anomolies, which are belived to be where waste pits are located. There is also pit information in the 2.0-2.5 meter and deeper images that are not clear in the 1.5 - 2.0 meter image, indicating pit depth information. The Channel-2 images also show pit locations, but are plagued by much more surface clutter, as would be expected from co-polarized data. The Channel- 2 images for 2.5 meters and greater depth alsơ contain high clutter, and are not shown since they convey no additional information. Future work will be to combine the co- and cross-polarized images to reduce clutter and enhance the reflection from pits.

\section{Magnetic (EM-61) and Conductivity (EM-31) Data Interpretation}

We have supported the data fusion effort by pre-processing the EM-61 induction sensor data. We used the USGS minimum-curvature (MINC) interpolation program to generate 


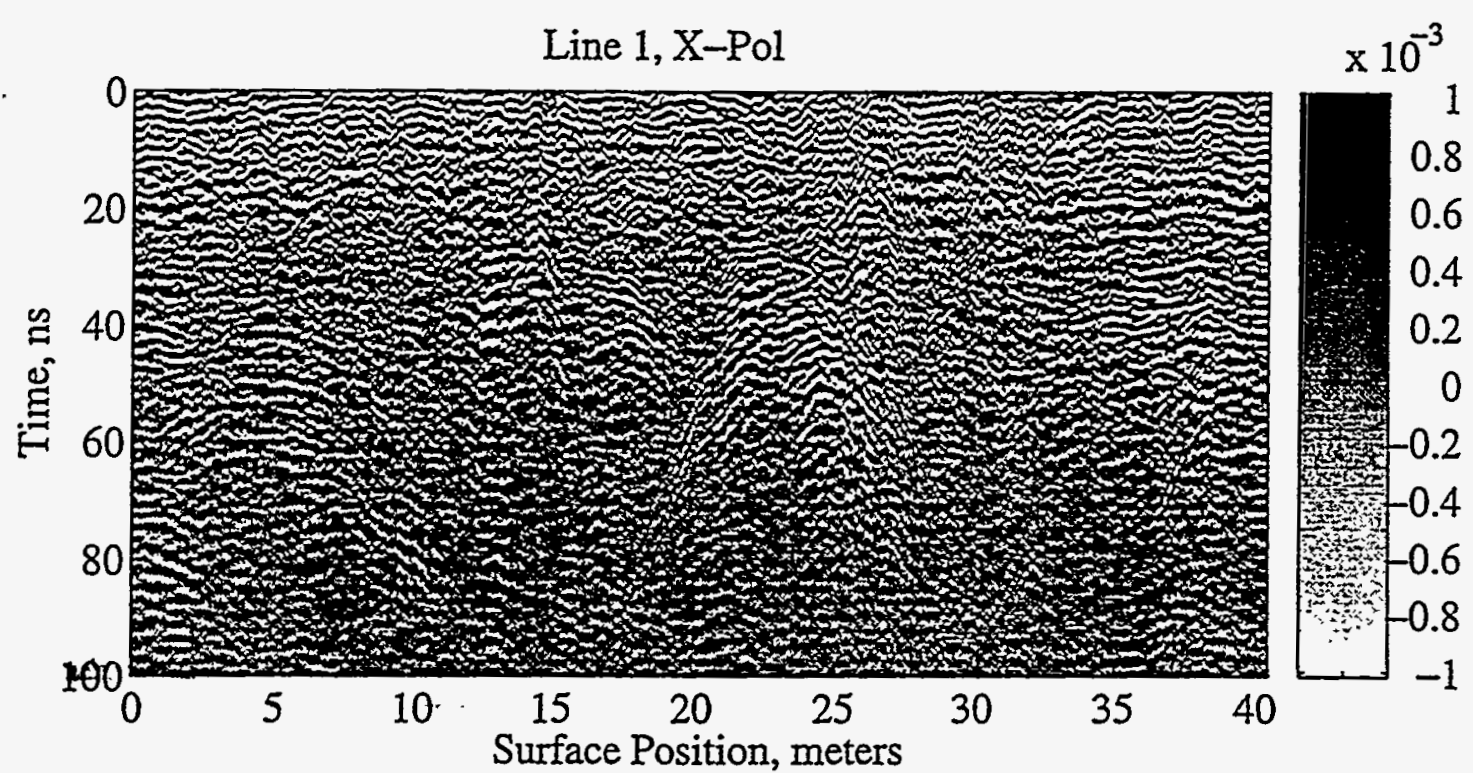

Figure 4: Typical line of data collected by the Coleman step-frequency radar in the glass-hole area at Brookhaven National Lab. The data line shown is line1, channel-1 (X-Pol). To illustrate the surface clutter effect, times $(t=0$ $\mathrm{ns})$ to $(t=100 \mathrm{~ns})$ are shown only. Data is clipped to $(\min =-.001, \max =$ $.001)$, no time-varying gain applied.

\section{Line-1, Channel-1}

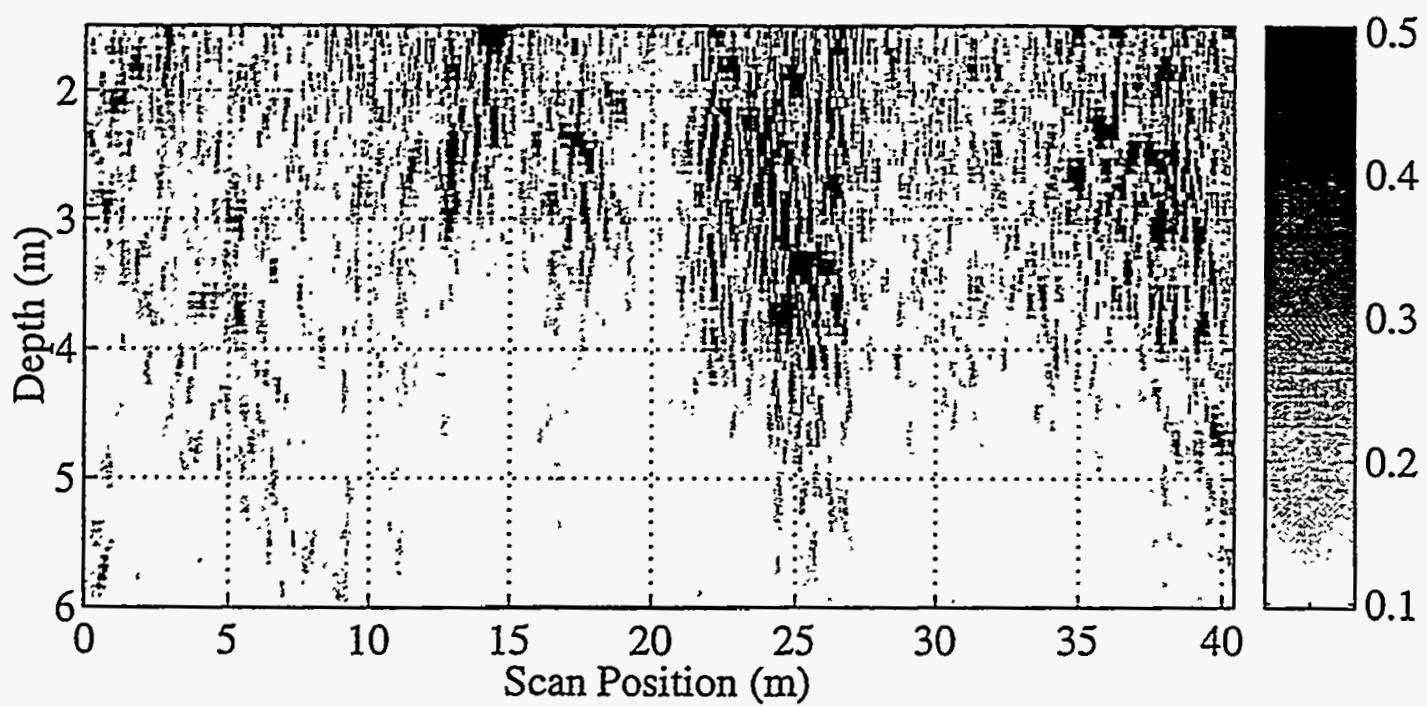

Figure 5: Backpropagation of Coleman SFR data shown in Figure 4. Backpropagation is from ( $z=1.5$ meters) to $(z=6.0$ meters $)$ to avoid eliminate surface clutter.

interpolated images from the EM-61 glass-hole pit data. The resulting EM-61 and EM31 images for the glass-hole area are shown in Figures 13 and 14. Note that the EM-61 and EM-31 provide complimentary information to each other and to the radar data. The EM-61 generally does a good job of detecting pits that contain metal, as well as providing several false detections (near the center of the image), due to random metallic surface

$$
\text { IIa }-5
$$




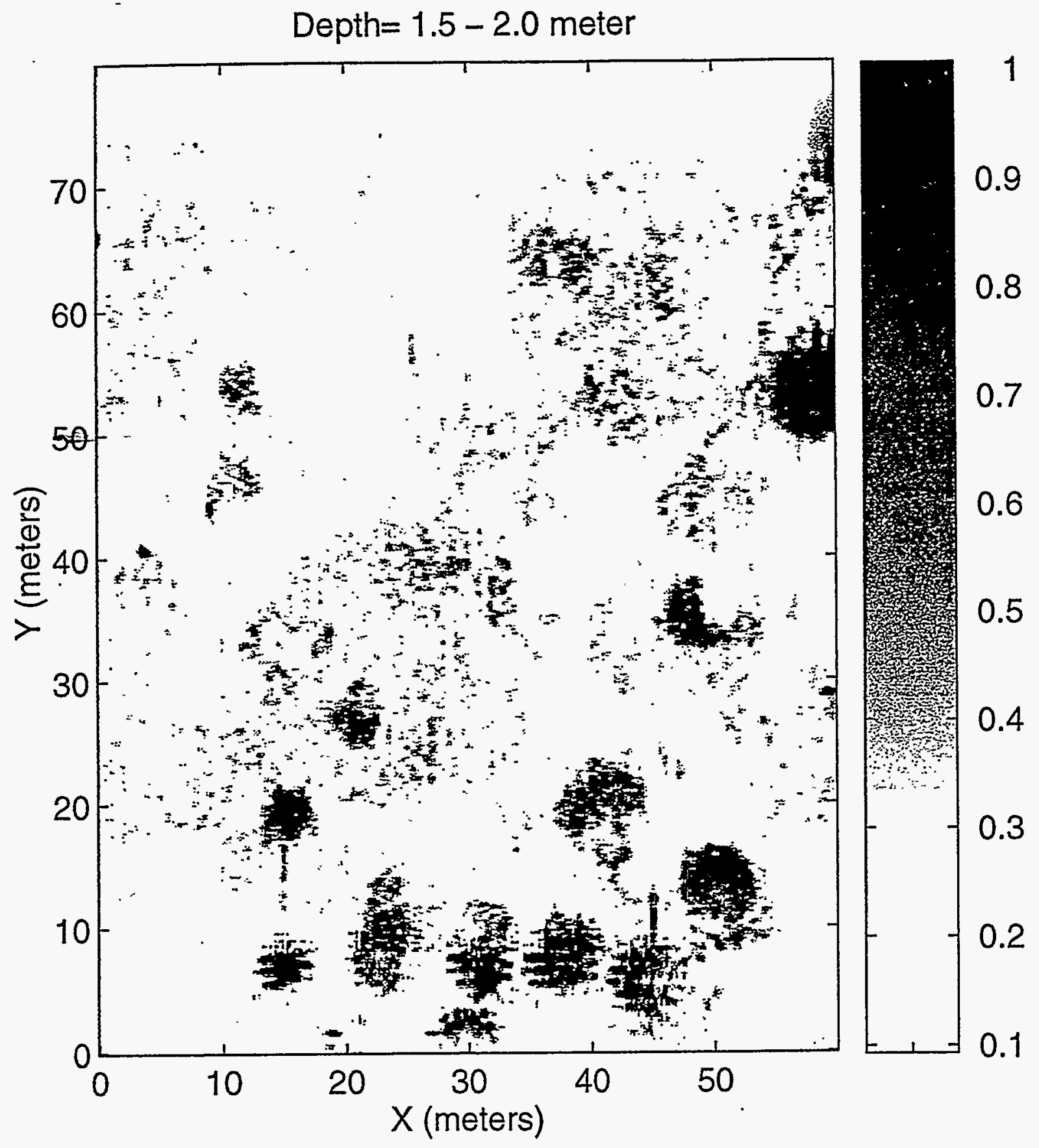

Figure 6: Constant-depth image of processed Coleman Step-Frequency radar data, Channel-1 (X-pol) for 1.5 - 2.0 meter depth. 


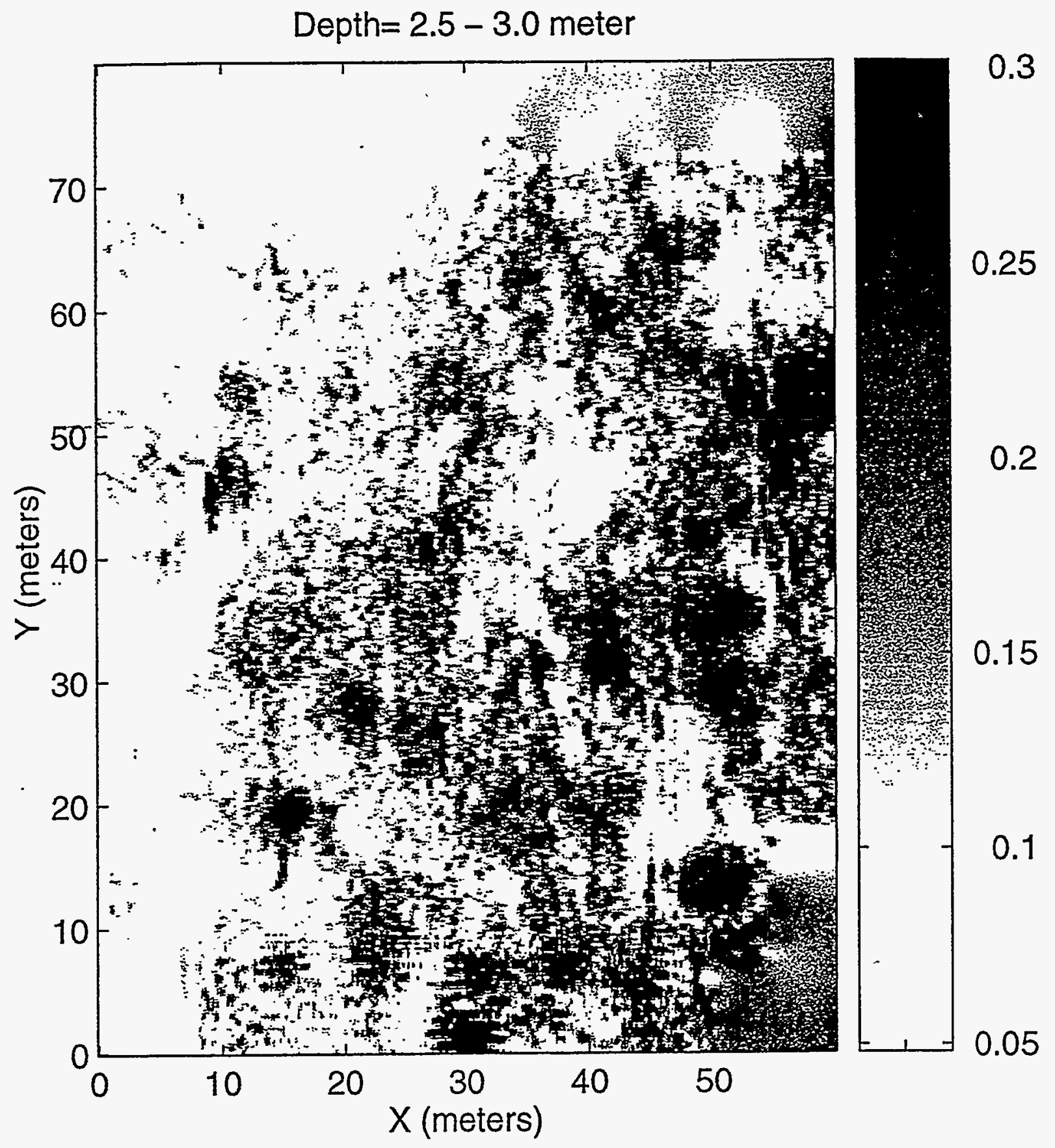

Figure 8: Constant-depth image of processed Coleman Step-Frequency radar data, Channel-1 (X-pol) 2.5-3.0 meter depth. 


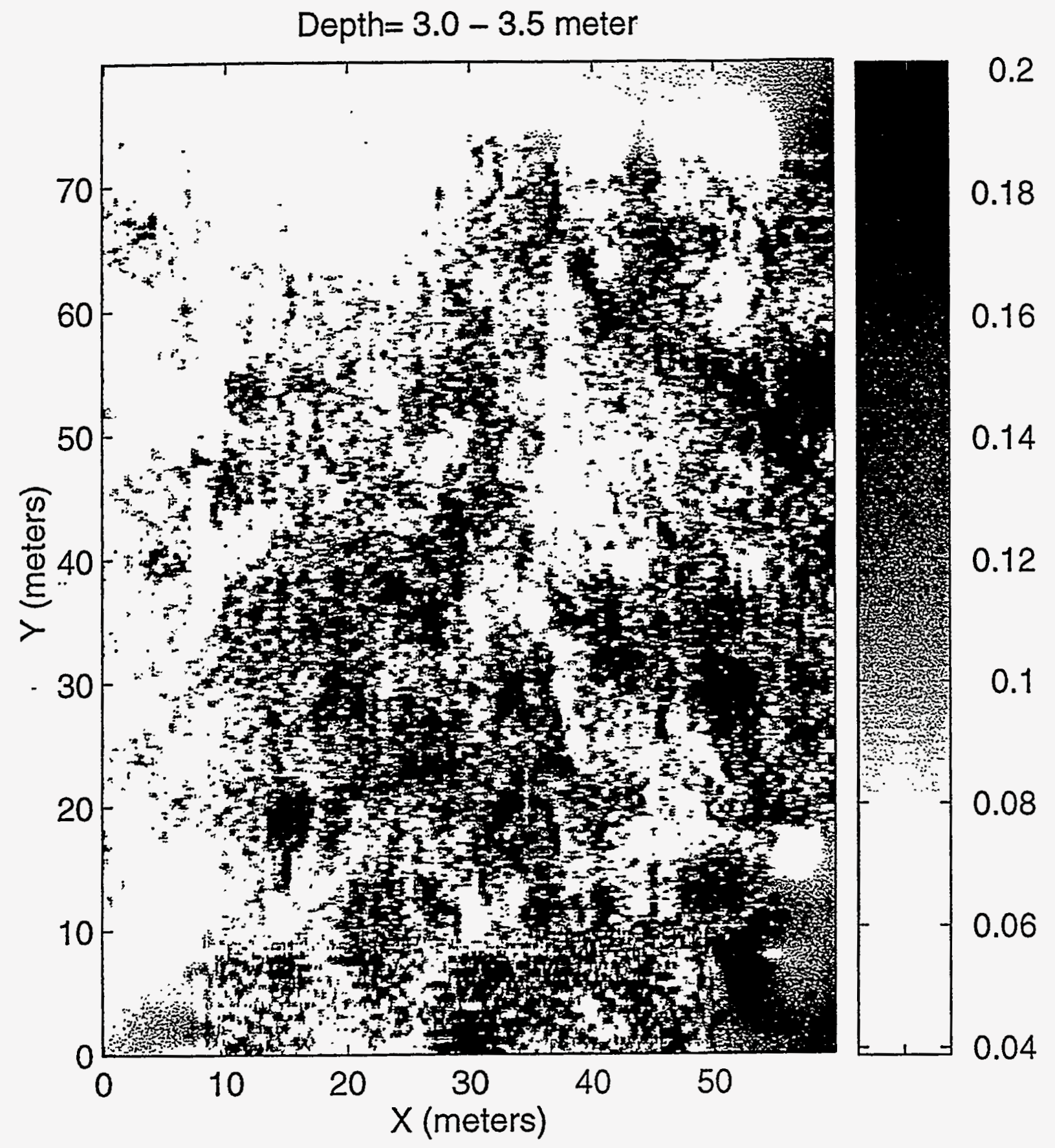

Figure 9: Constant-depth image of processed Coleman Step-Frequency radar data, Channel-1 (X-pol) 3.0 -.3.5 meter depth. 


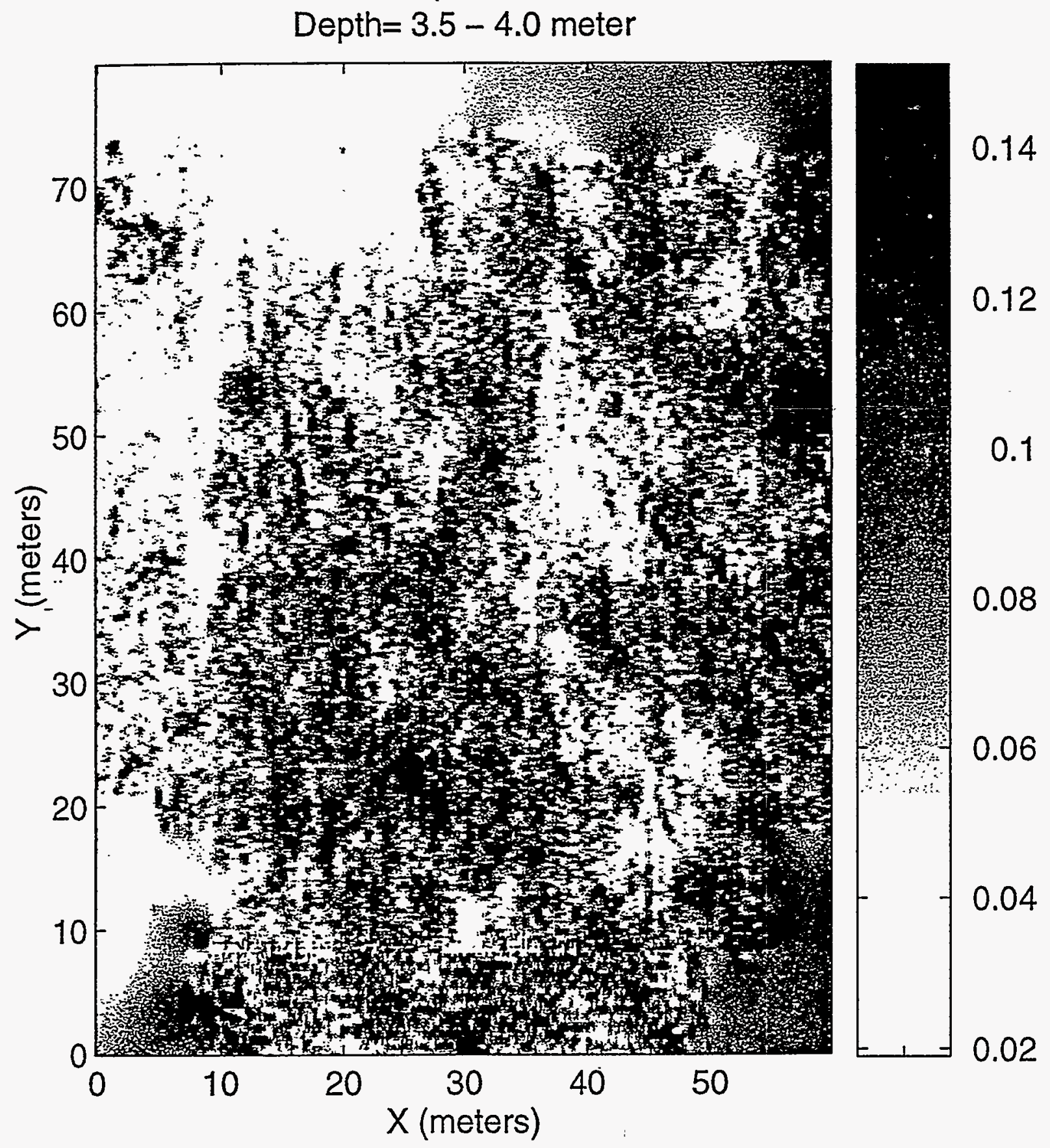

Figure 10: Constant-depth image of processed Coleman Step-Frequency radar data, Channel-1 (X-pol) 3.5 - 4.0 meter depth. 


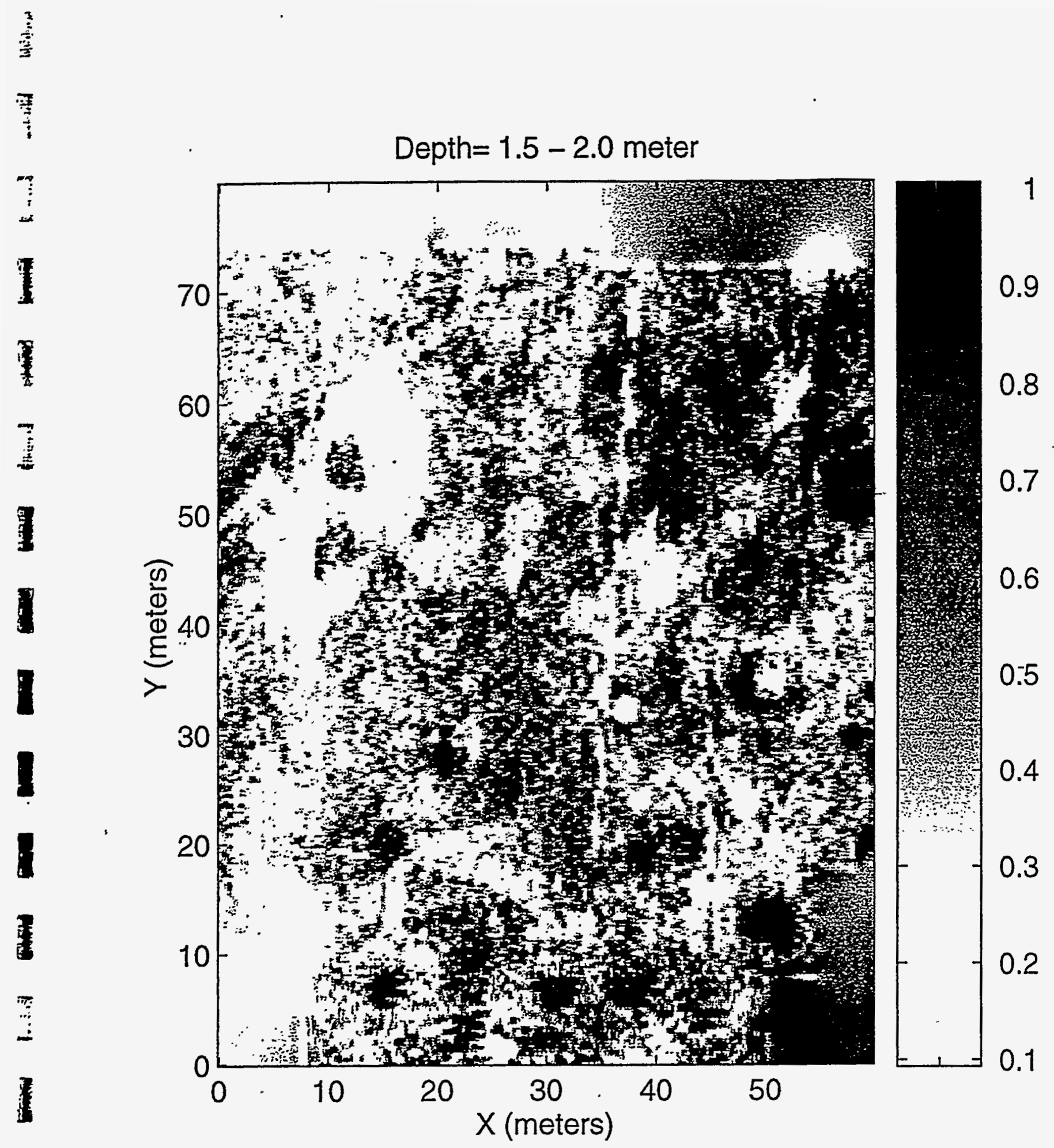

Figure 11: Constant-depth image of processed Coleman Step-Frequency radar data, Channel-2 (Co-pol) $1.5-2.0$ meter depth. 


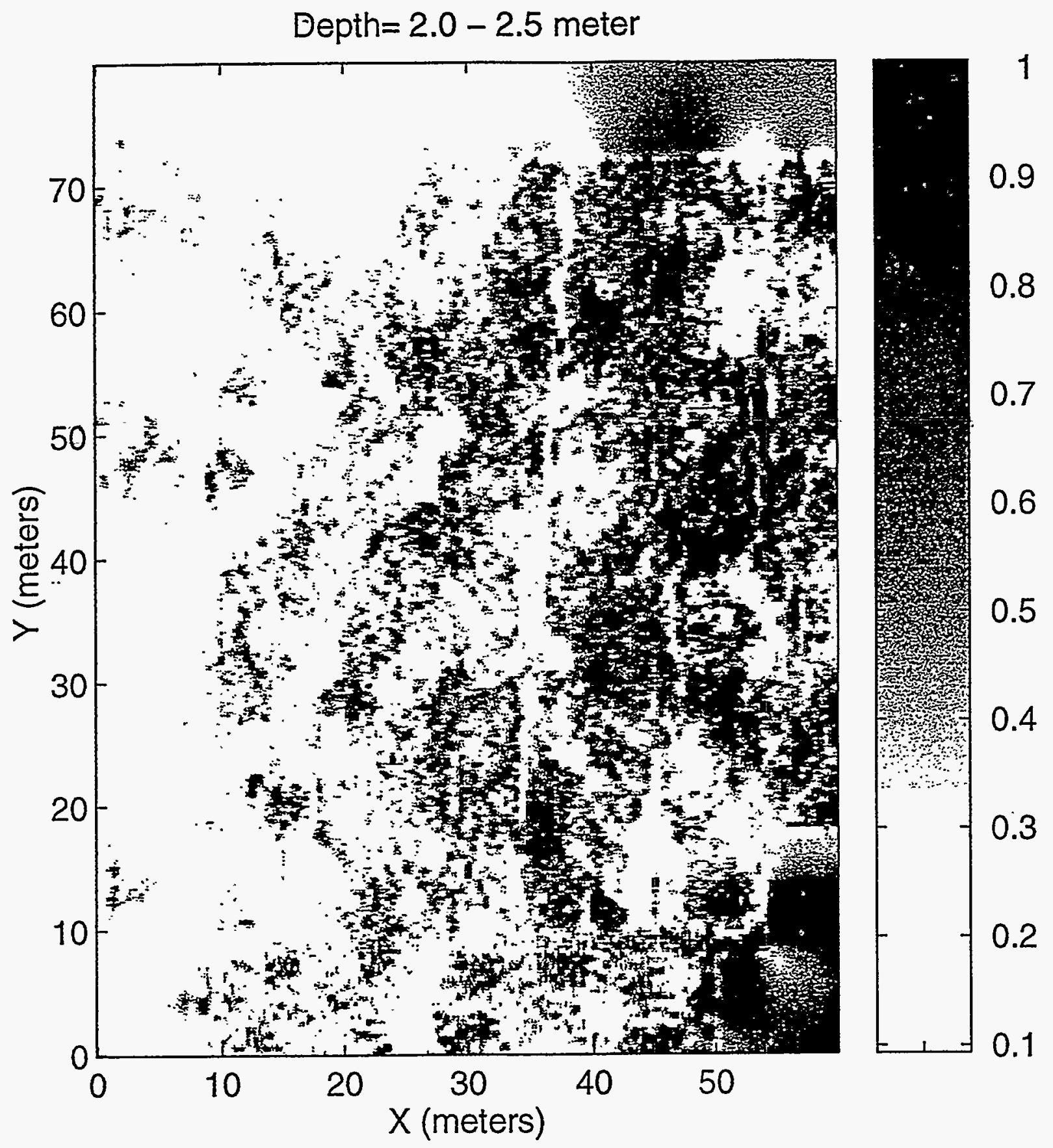

Figure 12: Constant-depth image of processed Coleman Step-Frequency radar data; Channel-2 (Co-pol) 2.0 - 2.5 meter depth. 
clutter. There are known pits detected by the EM-31 that are not detected by the EM-61, since they contain little or no metal. In general, we see excelent correlation between the EM-61, EM-31 and radar images.

\section{Detection and Fusion}

A pit detection algorithm has been developed to automatically detect possible waste pits in the EM-61, EM-31 and radar interpolated images. The detection algorithm generate size, position and orientation estimates, which are provided to the fusion processor as symbolic information, to be combined and correlated with similar information from other sensors. Figure 15 shows an interpolated image of the EM-61 data from the Brookhaven National Lab. glass-hole area. Overlaid on top of the image are ovals corresponding to the output of our detection algorithm. The size and orientation of the ovals correspond to the statistical size, position and orientation estimates of the pits.

The detection algorithm seems to capture the locations and sizes of most of the "bright spots" in the EM-61 interpolated image. The maximum pit size and the minimum spacing between pits are input as "tuning parameters" to the detection algorithm. As a result of the choice of input parameters, it is possible for there to be two or more detections for a single pit, or for two or more pits to be combined in a single detection. There are also false alarm detections. It is the job of the fusion algorithm to try to resolve the pits from the detections by combining with the detection results from the GPR and other sensors and any symbolic knowledge that can be incorporated into the problem.

The structure our proposed fusion processing system is presented in Figure 16. A key feature of our approach is that it allows feature detection to be performed separately on each sensor. The detected features are then passed as symbolic information to a higherlevel fused feature extraction step. Symbolic knowledge is incorporated, resulting in object classification and visualization. The fused information may also be used to control the sensors and provide additional information about classified objects.

\section{Conclusions}

Initial processing of the Coleman step-frequency radar data has shown that we can achieve high-quality reconstructions of calibration targets such as a pipe in air, as well as buried targets. Constant-depth images generated from the Brookhaven glass-hole data indicate that pit locations may be identified quite readily from the step-frequency radar data, and show good correlation with the EM-61 and EM-31 data. We plan to investigate the surface clutter problem, as well as extend our backpropagation to more general pseudoinverse formulation, as well as to include variable velocity and dispersion in the future. A pit detection algorithm has been developed to automatically detect objects in the EM-61 interpolated image data, in support of the sensor data fusion effort. 

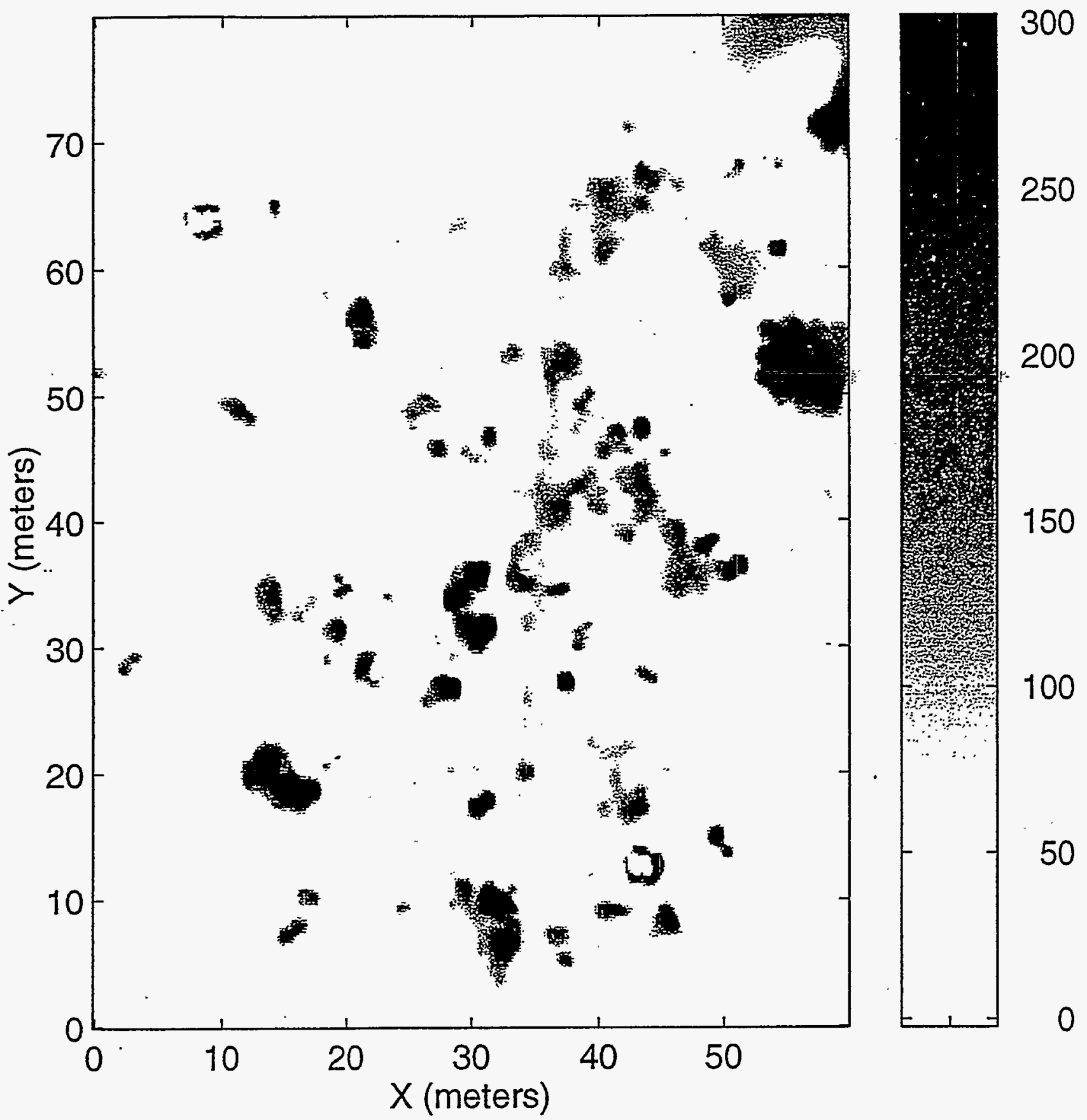

Figure 13: Minimum curvature processing applied to Geonics EM-61 metal detector data. 

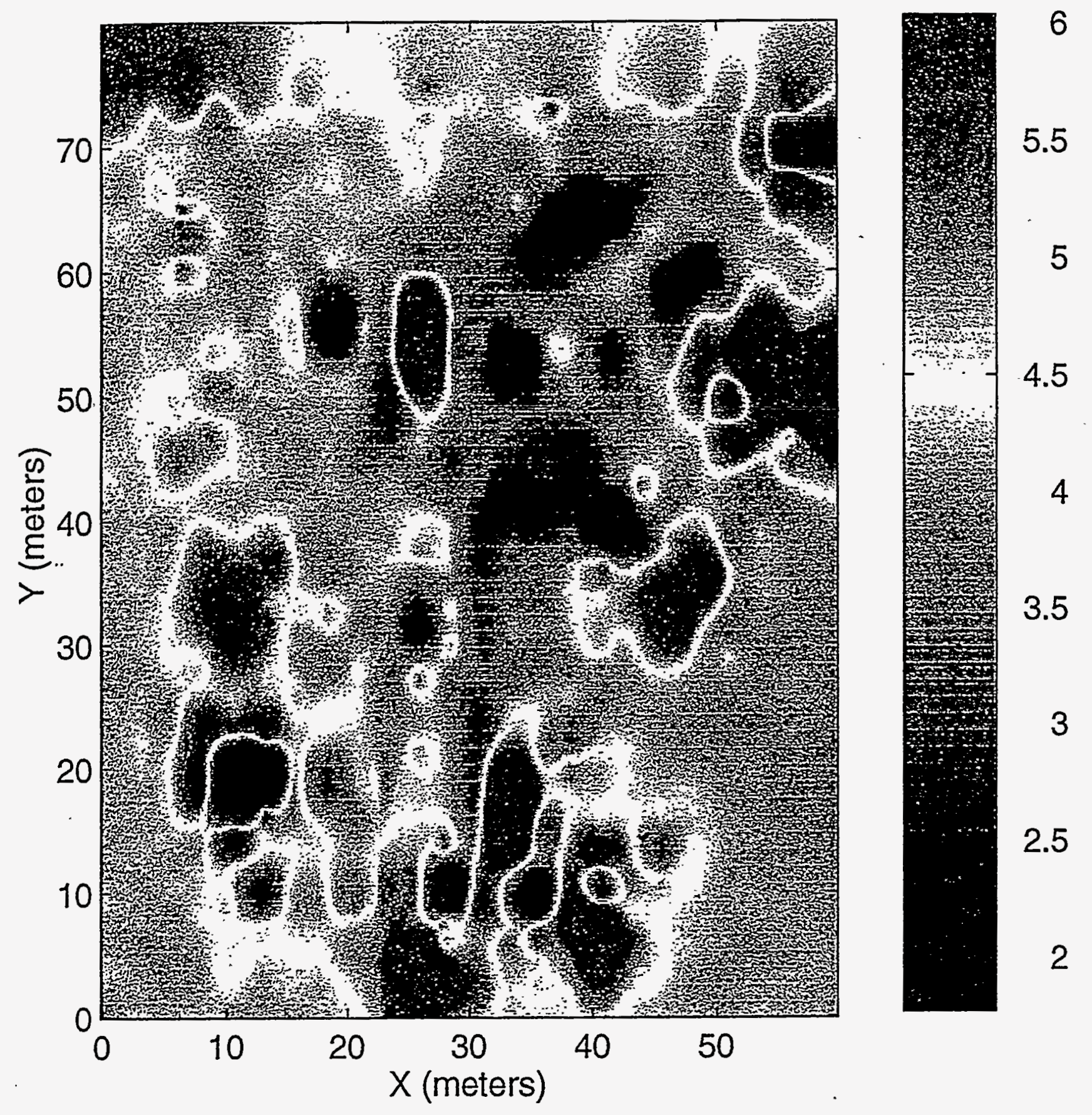

1

1

1

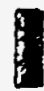

整

1

1

Figure 14: Minimum curvature processing applied to Geonics EM-31 conductivity sensor data, $\mathrm{X}$-direction profiles.

蕓

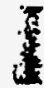



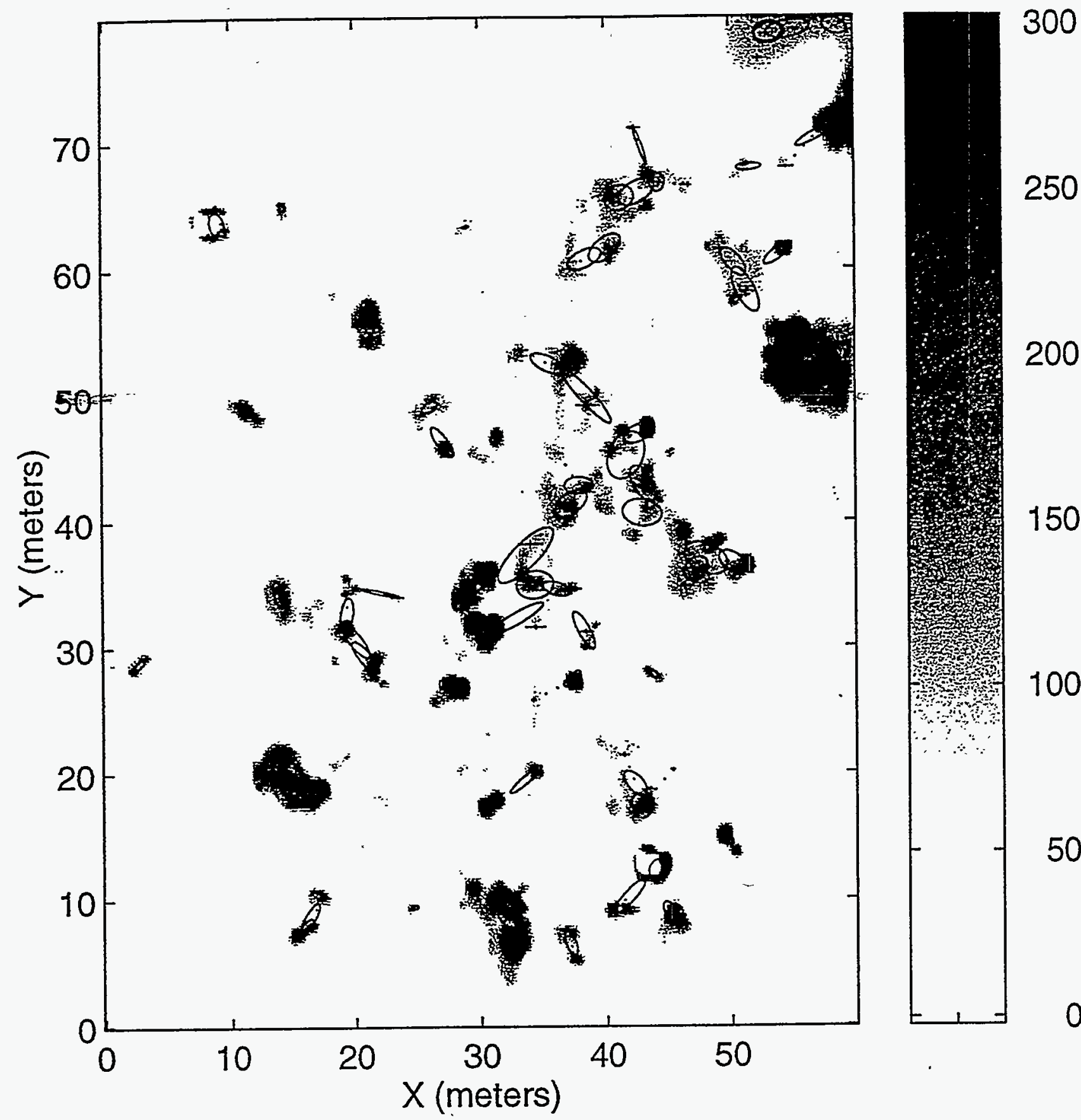

150

100

50

Figure 15: Interpolated EM-61 image of Brookhaven National Lab. glasshole area. Detection size, position and orientation estimates are indicated by ovals superimposed on top of interpolated image. 


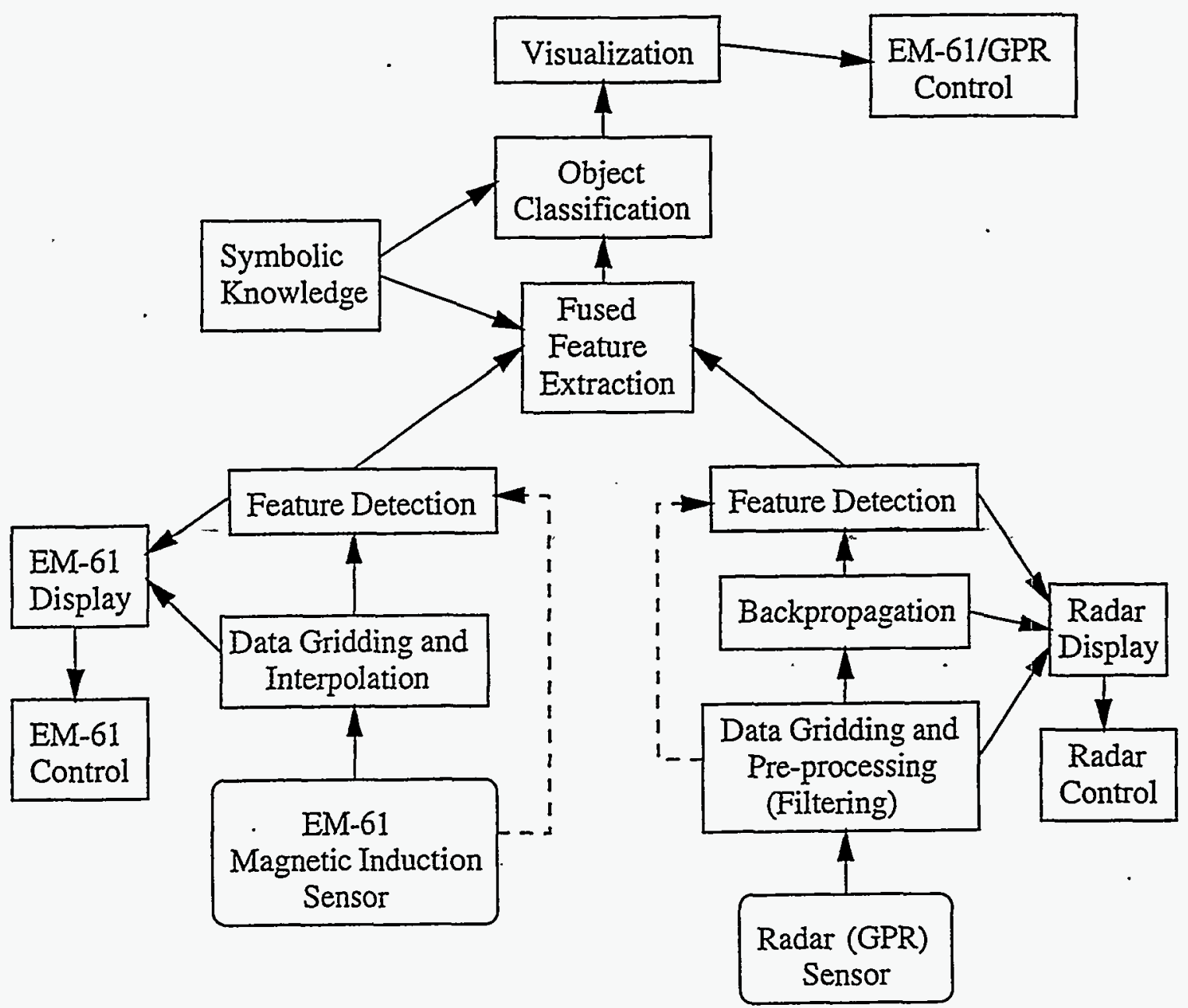

Figure 16: General Architecture of the Sensor Fusion System

\section{References}

[1] M. Kagalenko and W. H. Weedon, "Comparison of backpropagation and synthetic aperture imaging algorithms for processing gpr data," in IEEE Antennas and Propagation Society Internation Symposium, (Baltimore, MD), July 21-26 1996. Accepted for Presentation.

[2] "Calibration measurements final report," tech. rep., Coleman Research Corporation, Orlando, FL, Mar. 1995.

[3] "Preliminary survey findings," tech. rep., Coleman Research Corporation, Orlando, FL, May 1995.

[4] "Final hole locations and depth estimates," tech. rep., Coleman Research Corporation, Orlando, EL, July 1995. 


\title{
Chapter Ifb
}

\section{Tomographic Inversion of Time Domain Multi-Monostatic GPR Data}

\author{
R. W. Deming and A. J. Devaney \\ DEPARTMENT OF ELECTRICAL AND COMPUTER ENGINEERING \\ NORTHEASTERN UNIVERSITY, BOSTON, MA 02115
}

Final Report on Grant DE-FC07-95ID13395

May 14, 1996 


\section{$\frac{1}{2+\frac{1}{2}}$ \\ Tomographic Inversion of Time Domain Multi-monostatic GPR Data \\ R.W. Deming and A.J. Devaney \\ Department of EleCtrical and COMPUTER ENGineERING \\ NORTHEASTERN UNIVERSITY, BOSTON, MA 02115}

\section{Introduction}

Here we derive a method for subsurface imaging with ground penetrating radar (GPR) in a reflection geometry based on multi-monostatic time domain data measurements. Using this method we can estimate the complex index of refraction profile of the subsurface. The method assumes a linear scattering relation based on the Born approximation, and a farfield approximation for the incident and scattered fields. Also, the soil is assumed non-dispersive with attenuation properties which are frequency independent.

Using the approximate scattering relation, we first define a forward model giving the measured receiver voltage data in terms of the unknown index of refraction profile. We then derive an analytical expression for the pseudoinverse $[4,5]$ to the forward model, which is applied to the measured data yielding an image of the subsurface profile. This image represents the minimum $L^{2}$ norm solution to the forward model. The pseudoinverse operation is found to be a two step procedure consisting of (i) range gaining; (ii) Kirchhoff migration, a commonly used method in geophysical image processing $[7,8,9]$.

A computer program was designed to test the algorithm on experimental GPR data, and the results are presented in the final section below.

\section{Forward Model for Electromagnetic Scattering}

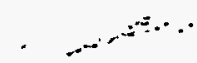

By probing the earth with electromagnetic wavefields we wish to estimate the electrical permittivity distribution in the underground region $z<0$ based on scattered field measurements at the surface $z=0$. The incident (probing) fields are generated by a pulsed time domain monostatic ground penetrating radar (GPR) system. The GPR survey is assumed to consist of a number of monostatic experiments, each corresponding to a different location of the transmitting/receiving

\section{IIb-1}


antenna on the ground surface. For the $j^{\text {th }}$ monostatic experiment, we have the following forward model relating the measured receiver terminal voltage $v\left(t_{m} ; j\right)$, sampled at time intervals $t_{m}$, to the unknown subsurface index of refraction profile $O\left(\mathrm{r}^{\prime}\right)$ under the Born approximation [2]:

$$
v\left(t_{m} ; j\right)=\int d^{3} r^{\prime} A^{2}\left(\hat{\mathrm{R}}_{\mathrm{j}}^{\prime}\right) F^{2}\left(R_{j}^{\prime}\right) p\left(t_{m}-2 n_{0} R_{j}^{\prime} / c\right) O\left(\mathrm{r}^{\prime}\right) .
$$

Here,

- $A^{2}\left(\hat{\mathrm{R}}_{\mathrm{j}}^{\prime}\right)$ is the radiation pattern of the antennas, assumed real and frequency independent, where $R_{j}^{\prime}=r^{\prime}-X_{j}, X_{j}$ is the position on the ground surface of the transmitting/receiving antenna, $\left.r=\left(x^{2} ; y^{2}\right) ; R_{j} \equiv \mid R_{j}^{r}\right]$ and $\overline{R_{j}^{r}}$ is a unit vector in the direction of $R_{j}^{r}$;

- $F\left(R_{j}\right)$ is the radial gain function which incorporates both geometrical wave spreading and soil attenuation;

- $p(t)$ is the transmitted pulse, expressed as voltage at the transmitting antenna terminals vs. time;

- $O(\mathrm{r})=k_{0}^{2}(\omega)-k^{2}(\mathrm{r}, \omega)$ is the object function which we seek to estimate, assumed real and frequency independent;

- $k(\mathrm{r}, \omega)=\omega \sqrt{\epsilon(\mathrm{r}, \omega) \mu_{0}}$ is the wavenumber in the underground, i.e. the soil with the embedded inhomogeneities, where $\epsilon=\epsilon^{\prime}+i \sigma / \omega$ is the complex permittivity, $\epsilon^{\prime}$ is the real dielectric constant, $\sigma$ is the conductivity, while the magnetic permeability $\mu$ is assumed to be equal to $\mu_{0}$ in a vacuum, and $k_{0}$. is the wavenumber of the homogeneous background soil medium;

- $n_{0}$ is the index of refration of the homogeneous background soil, while $c$ is the speed of light in a vacuum.

Eq.(1) can be rewritten in the operator notation

$$
v\left(t_{m} ; j\right)=G\left[O\left(\mathrm{r}^{\prime}\right)\right]
$$

where

- $O(\mathrm{r}) \in U$, where $U$ is the space of square integrable functions defined on $-\infty<(x, y)<\infty$, $-\infty<z<0$,

- $v\left(t_{m} ; j\right) \in W$, where $W$ is the product of two finite dimensional vector spaces of functions of the discrete variables $t_{m}$ and $j$,

- $G$ is a linear operator which maps $U$ into $W$. 


\section{Time Domain Data Inversion}

We wish to invert Eq.(2) to find the object function based on the data. Since we have only a finite amount of data, this equation is underdetermined, thus there are many. solutions for the object function which satisfy the data. We will use the pseudoinverse operator $[1,4,5]$ to find the unique minimum $L^{2}$ norm solution to Eq.(2)

$$
\hat{O}(\mathrm{r})=G^{T}\left(G G^{T}\right)^{-1} v\left(t_{m} ; j\right),
$$

where $G^{T}$ is the Hermetian adjoint of $G$.

Let us analytically expand Eq.(3). Given the vector space definitions, the Hermitian adjoint $G^{T}$ of $G$ defined by the inner product relation

$$
<G O, v>_{W}=<O, G^{T} v>_{U}
$$

is

$$
\begin{aligned}
G^{T}\left[\hat{v}\left(t_{m^{\prime}} ; j^{\prime}\right)\right] & \equiv \hat{O}\left(\mathrm{r}^{\prime}\right) \\
& =\sum_{j^{\prime}=1}^{J} \sum_{m^{\prime}=1}^{M} A^{2}\left(\hat{\mathrm{R}}_{\mathrm{j}^{\prime}}^{\prime}\right) F^{2}\left(R_{j^{\prime}}^{\prime}\right) p\left(t_{m^{\prime}}-2 n_{0} R_{j^{\prime}}^{\prime} / c\right) \hat{v}\left(t_{m^{\prime}} ; j^{\prime}\right) .
\end{aligned}
$$

Wie can define $\hat{v} \equiv\left(G G^{T}\right)^{-1} v$ as the filtered data, where $v$ is the rawr, unfiltered data, and $\left(G G^{T}\right)^{-1}$ is the filtering operation. Using the definitions of $G$ and $G^{T}$ from Eqs.(1) and (4), we then have the following relation between the filtered and unfiltered data:

$$
v\left(t_{m} ; j\right)=G G^{T}[\hat{v}]=\sum_{j^{\prime}=1}^{J} \sum_{m^{\prime}=1}^{M} \hat{v}\left(t_{m^{\prime}} ; j^{\prime}\right) B\left(t_{m}, t_{m^{\prime}} ; j, j^{\prime}\right)
$$

where

$$
B\left(t_{m}, t_{m^{\prime}} ; j, j^{\prime}\right)=\int_{z<0} d^{3} r^{\prime} A^{2}\left(\hat{\mathrm{R}}_{\mathrm{j}}^{\prime}\right) A^{2}\left(\hat{\mathrm{R}}_{\mathrm{j}^{\prime}}^{\prime}\right) F^{2}\left(R_{j}^{\prime}\right) F^{2}\left(R_{j^{\prime}}^{\prime}\right) p\left(t_{m}-2 n_{0} R_{j}^{\prime} / c\right) p\left(t_{m^{\prime}}-2 n_{0} R_{j^{\prime}}^{\prime} / c\right) .
$$

We could now solve for $\hat{O}$ using Eq.(3) by first numerically inverting Eq.(5), then applying Eq.(4). Instead, we wish to find an approximate solution which is more efficient to compute. To do this, we assume that the transmitted pulse is short relative to the sampling time interval. Simplified versions of both Eqs.(4) and (5) result. First we consider Eq.(4). Assuming the 
pulse $p(t)$ is shorter than the time sample interval, the $m^{\prime}$ summation has a contribution only at $t_{m^{\prime}}=2 n_{0} R_{j^{\prime}} / c$, resulting in the approximation

$$
G^{T}\left[\hat{v}\left(t_{m^{\prime}} ; j^{\prime}\right)\right] \approx C^{\prime} \sum_{j^{\prime}=1}^{J} A^{2}\left(\hat{\mathrm{R}}_{j^{\prime}}\right) F^{2}\left(R_{j^{\prime}}\right) \hat{v}\left(2 n_{0} R_{j^{\prime}} / c ; j^{\prime}\right)
$$

where $C^{\prime}$ is a constant which is approximately independent of $j^{\prime}$ and $m^{\prime}$.

Next we simplify Eq.(5). If the transmitted pulse $p(t)$ has short duration relative to the time sample spacing, it can be shown [2] that the diagonal terms $B\left(t_{m}=t_{m^{\prime}} ; j=j^{\prime}\right)$ dominate. Therefore Eq.(5) can be approximated as

$$
\hat{v}\left(t_{m} ; j\right)=\left(G G^{T}\right)^{-1}[v] \approx v\left(t_{m} ; j\right) / B\left(t_{m} ; j\right)
$$

where

$$
B\left(t_{m} ; j\right)=B\left(t_{m}\right)=\int_{z<0} d^{3} r^{\prime} A^{4}\left(\hat{\mathrm{r}}^{\prime}\right) F^{4}\left(r^{\prime}\right) p^{2}\left(t_{m}-2 n_{0} r^{\prime} / c\right) .
$$

We seek a further simplification for the function $B$. Converting to spherical coordinates $\mathbf{r}^{\prime}=$ $\left(r^{\prime}: \Omega_{r^{\prime}}\right)$, where $\Omega_{r^{\prime}}$ is the solid angle $\mathrm{r}^{\prime}$ makes relative to the coordinate axis,

$$
B\left(t_{m}\right)=\frac{1}{2} \int_{0}^{\infty} d r^{\prime} r^{\prime 2} F^{4}\left(r^{\prime}\right) p^{2}\left(t_{m}-2 n_{0} r^{\prime} / c\right) \int d \Omega_{\tau^{\prime}} A^{\prime 4}\left(\Omega_{r^{\prime}}\right)
$$

where $A^{\prime}$ is the directional gain expressed as a function of the solid angle $\Omega_{r^{\prime}}$. When the duration of the pulse $p(t)$ is very short relative to the rate of change of $r$ and $F(r)$, Eq.(9) becomes

$$
\begin{aligned}
B\left(t_{m}\right) & \approx \frac{1}{2}\left(\frac{c t_{m}}{2 n_{0}}\right)^{2} F^{4}\left(\frac{c t_{m}}{2 n_{0}}\right) \int_{0}^{\infty} d r^{\prime} p^{2}\left(t_{m}-2 n_{0} r^{\prime} / c\right) \int d \Omega_{r^{\prime}} A^{\prime 4}\left(\Omega_{r^{\prime}}\right) \\
& =C\left(\frac{c t_{m}}{2 n_{0}}\right)^{2} F^{4}\left(\frac{c t_{m}}{2 n_{0}}\right),
\end{aligned}
$$

where $C$ is a constant independent of $m$. Thus Eq.(8) becomes

$$
\hat{v}\left(t_{m} ; j\right)=\left(G G^{T}\right)^{-1}[v] \approx \frac{v\left(t_{m} ; j\right)}{C\left(c t_{m} / 2 n_{0}\right)^{2} F^{4}\left(c t_{m} / 2 n_{0}\right)} .
$$

Substituting Eqs.(11) and (7) into Eq.(3),

$$
\hat{O}(\mathfrak{r})=K \sum_{j^{\prime}=1}^{J} A^{2}\left(\hat{\mathrm{R}}_{j^{\prime}}\right) \frac{v\left(2 n_{0} R_{j^{\prime}} / c ; j^{\prime}\right)}{R_{j^{\prime}}^{2} F^{2}\left(R_{j^{\prime}}\right)},
$$


where $K$ is a constant. Eq.(12) shows that if the transmitted pulse is shorter than the sampling time interval, the pseudoinverse image is proportional to the sum of $K$ different subimages, each corresponding to a single experiment. A particular subimage can be quickly computed by evaluating the corresponding data return along a radius $r$ at $t=2 n_{0} r / c$, then multiplying this data by $1 /\left[r^{2} F^{2}(r)\right]$, and finally by sweeping this one dimesional function across the half-space while multipying by the radiation pattern $A(\hat{\mathrm{r}})$. Each subimage is shifted in order to correspond to its transmitter/receiver location. This process is equivalent to range gaining followed by Kichhoff migration $[7,8,9]$, or "hyperbola summing". A typical subimage (two dimensional slice) is illustrated in Fig. 1.

We can find an alternate form for the object function solution. Using statistical methods, the radial gain function $F(R)$ can be estimated directly from the unprocessed data by the relation [2]

$$
F^{2}(R) \propto \frac{\sigma_{N}\left(2 n_{0} R / c\right)}{R}
$$

where $\sigma_{N}(t)$ is the standard deviation of the return data as a function of time, computed by treating the return from each monostatic experiment as a random process. Substituting this expression into Eq.(12),

$$
\hat{O}(\mathrm{r})=K \sum_{j^{\prime}=1}^{J} A^{2}\left(\hat{\mathrm{R}}_{\mathrm{j}^{\prime}}\right) \frac{v\left(2 n_{0} R_{j^{\prime}} / c ; j^{\prime}\right)}{R_{j^{\prime}} \sigma_{N}\left(2 n_{0} R_{j^{\prime}} / c\right)}
$$

Since the factor $1 / R_{j^{\prime}}$ in this expression blows up at small $R_{j^{\prime}}$ values, this factor may need to be tapered off in some manner near $R_{j^{\prime}}=0$ when analyzing experimental data. This tapering is justified since antennas do not behave like point sources in the near field.

\section{Computed results for experimental data}

The approximate time domain reconstruction algorithm described in Section 3 was tested on experimental data obtained from a step-frequency radar system manufactured by Georadar, Inc. This system uses 450 frequency steps in the $100 \mathrm{Mhz}$ to $1 \mathrm{GHz}$ range, and logarithmic spiral antennas approximately 15 inches in diameter which generate circularly polarized fields. Although the system is technically bistatic, we use the monostatic approximation for simplicity.

The test site consists of a pit filled with fairly wet soil and rocks, in which two steel pipes are buried. A $4^{\prime \prime}$ diameter pipe is buried at approximately $(x, z)=(2.5,-0.6)$ meters, and a

$$
\text { IIb-5 }
$$


$6^{\prime \prime}$ diameter pipe is buried approximately $(x, z)=(2.0,-1.0)$ meters. The survey consists of 90 monostatic experiments, spaced approximately uniformly along a line 4 meters long running perpendicular to the pipe axes. In each experiment, the step-frequency data is initially Fourier transformed to give a time domain return consisting of 70 samples at $4.5 e-10$ second intervals. The time domain returns for all experiments are stacked, and shown in Fig.2. From Fig.2, the $4^{\prime \prime}$ pipe at $(2.5,-0.6)$ appears as a hyperbola, while the $6^{\prime \prime}$ pipe is not clearly visible. Based on the shape of the hyperbola, and the known horizontal spacing between experiments, the propagation velocity in the soil is determined to be roughly $2.0 e 8 \mathrm{~m} / \mathrm{s}$. The radiation pattern, also estimated from the hyperbola, is found to be roughly $\cos ^{2} \theta$, where $\theta$ is measured from the $-z$ axis.

Fig. 3 shows the reconstructed image, as calculated from Eq.(13). Since the antennas cannot be accurately modeled as point sources, the $1 / R$ factor in Eq.(13) is actually tapered off in an adhoc manner for small $R$ values to avoid the singularity. In Fig. 3 the $4^{\prime \prime}$ pipe at $(2.5,-0.6)$ appears prominantly, and the $6^{\prime \prime}$ pipe at $(2.0,-1.0)$ is somewhat visible. The discrepancy between the known location of the pipes and there apparent position in the image is caused by an amount of uncertainty in the spacing between adjacent experiments.

Another survey was then conducted, as before, where the measurements are moved several meters down the axis' of the pipes. The reconstructed image is shown in Fig.4, and is seen to be very good, with both the $4^{\prime \prime}$ and the $6^{\prime \prime}$ pipes visible.

Our algorithm was implemented on one final data set, using a "Sensors and Software" brand pulsed time domain radar at a site in Idaho. We show the stacked, range gained, returns in Fig.5, and the processed image in Fig.6. The processed image will be checked against independent information as it becomes available. 


\section{References}

[1] Bertero M., DeMol C., Viano G.A., 1980, The stability of inverse problems, in Baltes H.P., Ed., Inverse scattering problems in optics: Topics in current physics, 20, Springer-Verlag, 161-214.

[2] Deming R.W., New tomographic methods for subsurface imaging with GPR: Ph.D thesis, Northeastern University, in progress - to be completed Sept. 1996.

[3] Devaney A.J., 1984, Geophysical diffraction tomography: IEEE trans. on Geosci. and Remote Sensing, GE-22, no.1, 3-13.

[4] Jain A.K., 1986, Fundamentals of digital image processing: Prentice Hall.

[5] Natterer F., 1986, The mathematics of computerized tomography: Wiley.

[6] Press W.H., Teukolsky S.A., Vetterling W.T., Flannery B.P., 1992, Numerical recipes in FORTRAN: the art of scientific computing, second edition: Cambridge University Press.

[7] Robinson E.A., 1983, Migration of geophysical data, International Human Resources Developement Corporation, Boston.

[8] Schneider W.S., 1978, Integral formulation for migration in two and three dimensions: Geophysics, 43, pp. 49-76.

[9] Tarantola A., 1984, Linear Inversion of Seismic Reflection Data: Geophys. Prosp., 32, pp. 998-1015.

[10] Tarantola A., 1984, Inversion of seismic reflection data in the acoustic approximation: Geophysics, 49, no.8, pp.1259-66. 


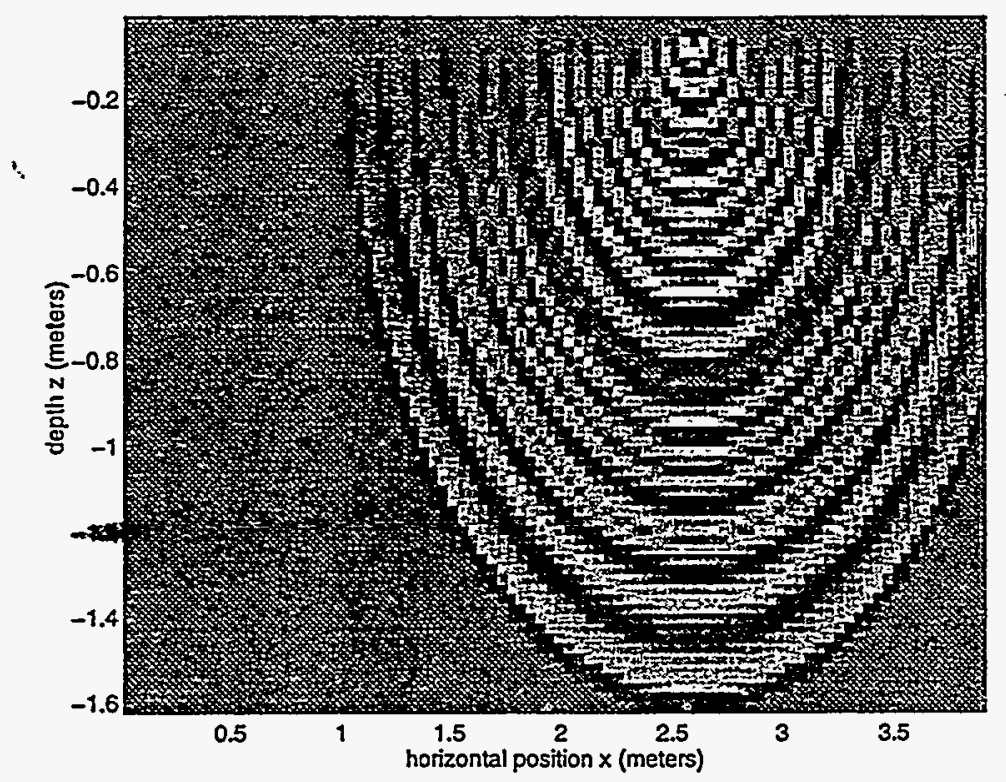

Fig.1 - Typical subimage for time domain imaging.

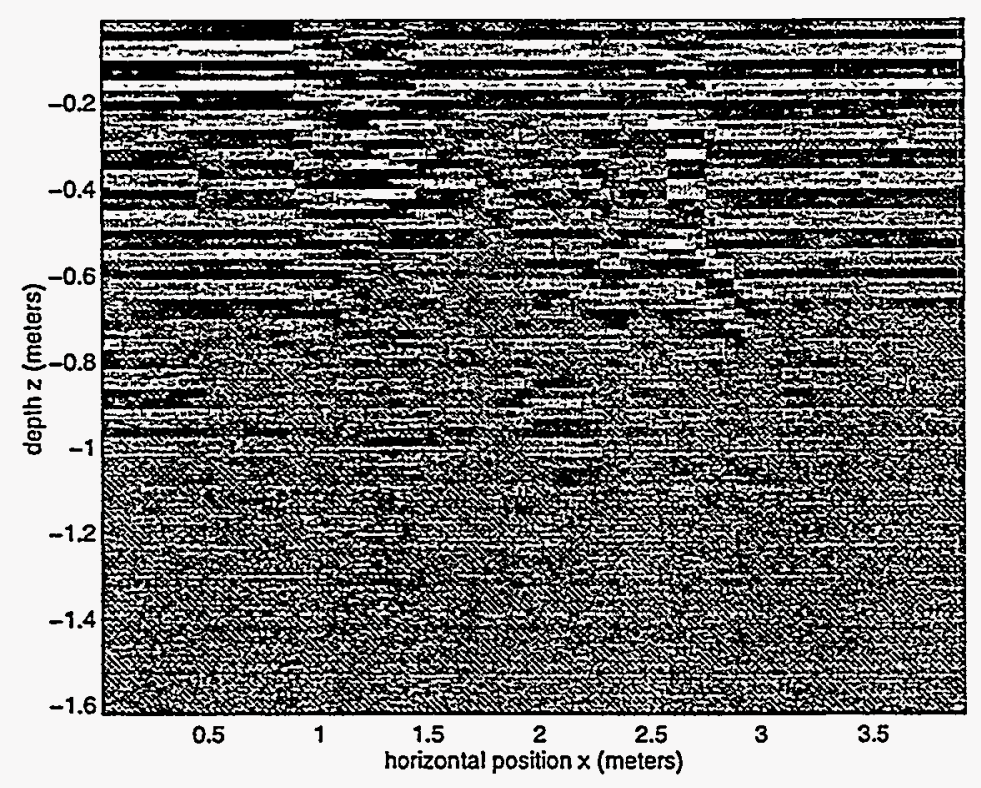

Fig.2 - Stacked time domain returns from 90 experiments conducted with an actual GPR system. 


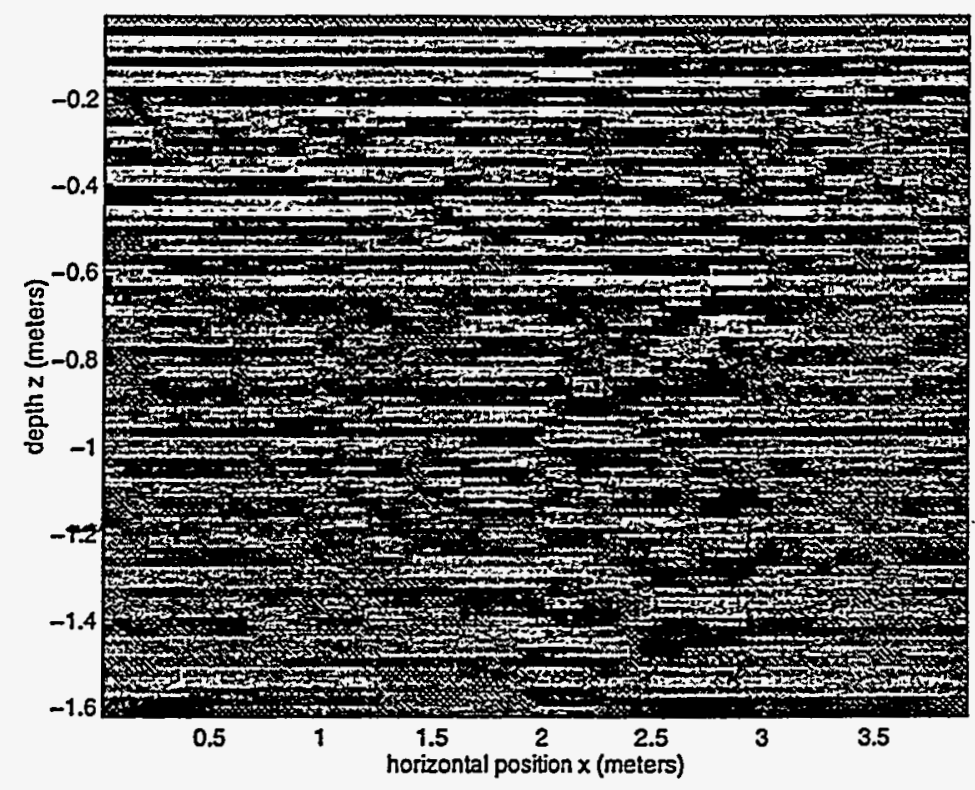

Fig.3 - Time domain reconstruction from first experimental survey. A $4^{\prime \prime}$ pipe at $(x, z)=$ $(2.5,-0.6)$ meters is clearly visible, while a $6^{\prime \prime}$ pipe at $(x, z)=(2.0,-1.0)$ meters is barely visible. Location discrepancies due to irregular spacing of experiments during collection.

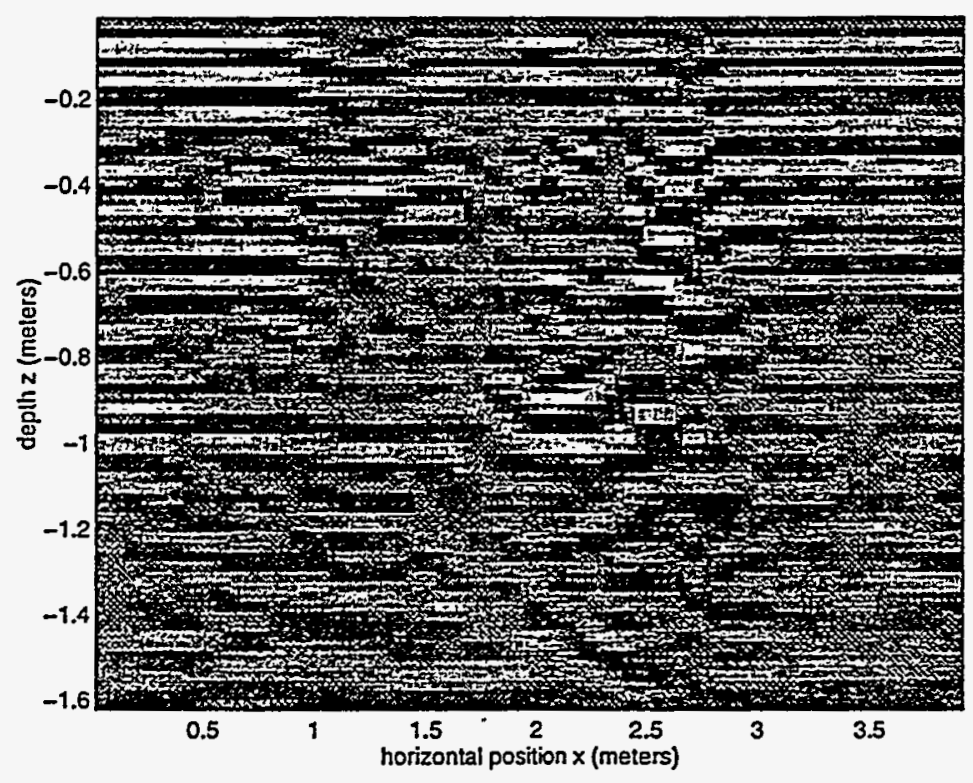

Fig.4 - Time domain reconstruction from second experimental survey. Both a $4^{\prime \prime}$ pipe at $(x, z)=(2.5,-0.6)$ meters and a $6^{\prime \prime}$ pipe at $(x, z)=(2.0,-1.0)$ meters are visible.

$$
\text { IIb-9 }
$$




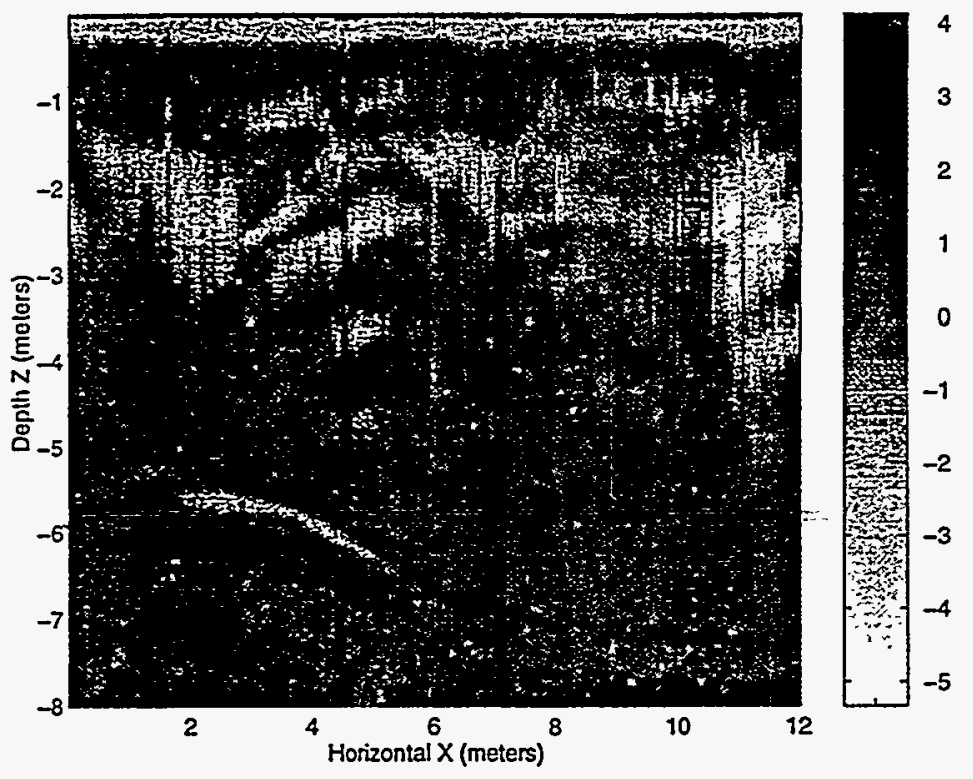

Fig.5 - Stacked and range.gained data from "Sensors and Software" brand pulsed GPR system.

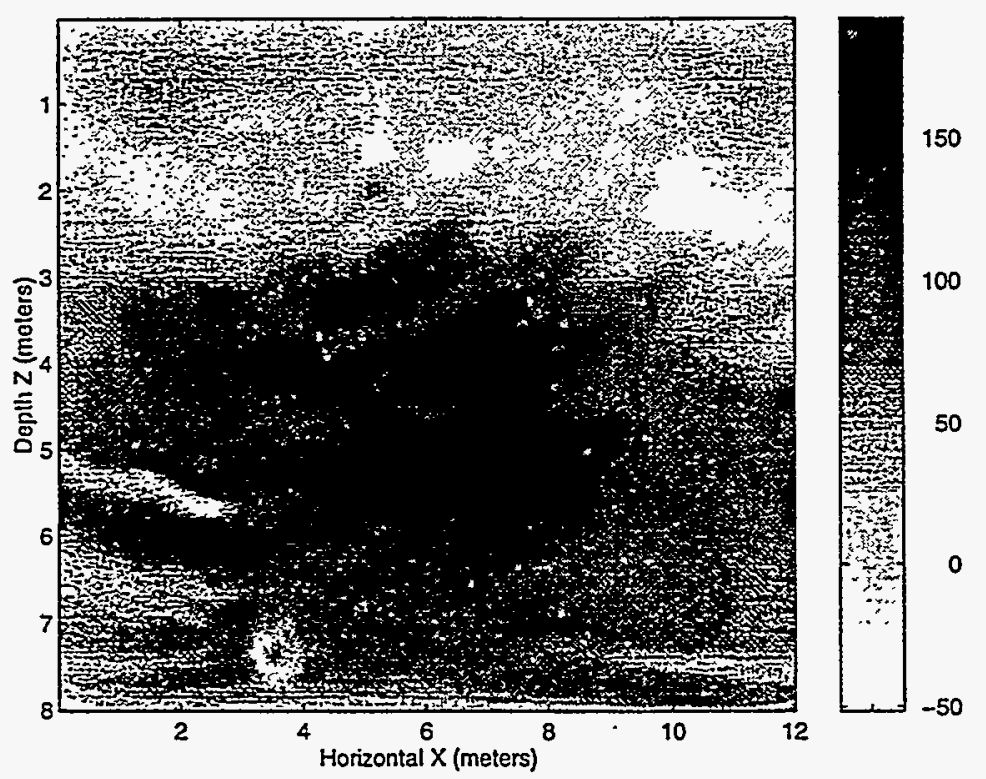

Fig.6 - Time domain reconstruction from "Sensors and Software" brand pulsed GPR system. 


\title{
Chapter III
}

\section{Object-Based Localization of Buried Waste Containers Using High Resolution Array Processing Techniques}

\author{
ERIC L. Miller and AdNan SahiN \\ Center for EleCtromagnetics Research \\ DEPARTMENT OF ELECTRICAL AND COMPUTER ENGINEERING \\ Northeastern UnIVERsity, Boston, MA 02115
}

FINAL REPort on GRANT DE-FC07-95ID13395

May 14, 1996 


\title{
Object-Based Localization of Buried Waste Containers Using High Resolution Array Processing Techniques
}

\author{
Prof. Eric L. Miller \\ Mr. Adnan Sahin \\ Center for Electromagnetics Research, \\ 235 Forsyth Building, 360 Huntington Ave. \\ Northeastern University, Boston, MA 02115 \\ Telephone: (617) 373-8386 \\ Telefax - $(-617) 373-8627$ \\ email: elmiller@cdsp.neu.edu
}

May 13, 1996

\begin{abstract}
In this report we explore the use of high-resolution array processing techniques for the detection and localization of buried waste containers. A ground penetrating radar (GPR) scenario is considered where data is collected over a linear receiver array positioned at the air-earth interface. We have modified the high-resolution algorithm MUSIC to explicitly account for the near-field physics and solved the localization problem in two ways. The first method uses the MUSIC algorithm in a matched field processing scheme and determines both the bearing and the range of the containers directly. Under the second approach, the sensor array is divided into non-overlapping subarrays. The targets are assumed to lie in the far-field of each subarray ensuring a planar wavefront over each partition. Conventional plane wave MUSIC is used to find the direction of arrival (DOA) of the waves over each subarray and the locations of the targets are determined by triangulation of the DOAs. Using simulated data we demonstrate that these techniques are quite useful for the detection and localization of buried metallic drums. The favorable detection results are shown to hold over a wide range of soil conditions and signal to noise ratios.
\end{abstract}

\section{Introduction}

In recent years there has been considerable interest in the use of ground penetrating radar (GPR) for the non-invasive detection, localization, and classification of buried objects. One of the most promising application areas for this technology is hazardous waste remediation. The primary difficulty associated with the use of GPR in this context is the extraction of the object.information from the raw data. Typical algorithms first generate a pixel-by-pixel map of the subsurface and subsequently post-process these images to detect the targets of interest. There are however a variety of difficulties associated with this image-then-detect approach. First, the image formation process is highly ill-posed and therefore quite sensitive to noise. While the ill-posedness can be offset through regularization, most regularization methods result in overly smooth images thereby making object detection all the more difficult. Second, the processing of GPR data requires that some level of the scattering physics be incorporated into the algorithms. Linearized scattering models based on the 
Born or Rytov approximations [5,6] are inappropriate for many practical applications (e.g. detection of high contrast, metallic objects) while exact, nonlinear scattering models lead to computationally intensive algorithms.

In this work, we circumvent these difficulties by exploiting the fact that for the waste remediation problem the principle, underlying objective is the localization of objects with known structures, eg. 55 gallon drums. Thus, we reparameterize the problem from one of determining a large number of pixel values to one of identifying a small parameter vector containing the coordinates of the objects in the field of view. By constraining the degrees of freedom in this manner, ill-posedness is no longer an issue and the resulting algorithms prove to be highly robust to noise. Additionally, modeling the targets of interest as simple shapses, spheres, etc.) allows for the incorporation into the processing of highly efficient exact scattering models based on a $T$-matrix formulation $[2,11]$ of the physics. Finally, the inherent array structure of GPR measurement geometry (Fig. 1) suggests that the high resolution array processing techniques so popular in the signal processing community would be well suited for the subsurface detection problem.

Of particular interest for most of this report is the localization of a single, metallic cylindrical object buried in a homogeneous, lossy background as a model for the waste container detection scenario. Using two processing methods based on the Multiple Signal Classification (MUSIC) algorithm [12], we can estimate the center of the object to within a fraction of a radius for signal to noise ratios down $0 \mathrm{~dB}$ and over a wide range of soil characteristics from $20 \%$ moist San Antonio clay loam to dry Puerto Rico clay loam. The results are obtained using a single frequency, GPR setup with plane wave illumination and an array of 16 point receivers stretching over at most a 75 $\mathrm{cm}$ aperture.

The remainder of the report is organized as follows. In Section 2, a brief description of the problem geometry is given. The MUSIC algorithm is reviewed in Section 3. In Sections 4 and 5, we discuss the algorithms developed for the GPR problem. Numerical examples are presented in Section 6 for the case where a single object is buried in the array's field of view. In Section 7, we discuss recent work in extending these methods to the multi-object case. Finally, directions for future research and conclusions are presented in Section 8.

\section{Problem Geometry}

The ground penetrating radar (GPR) measurement scheme depicted in Fig. 1, is considered in this report. The objects are buried in the lower half space for which the soil's electrical characteristics (relative permittivity and conductivity) are assumed known. A TM-polarized plane wave, $E_{i}(r, t)$, impinges on the objects, inducing surface and volume currents which in turn radiate a scattered field, $E_{s}(\mathrm{r}, t)$. For simplicity, in this work we assume that the number of objects, their material properties, and their shapes are known a priori. The scattered electric field from these objects is spatially sampled by a uniformly spaced, linear array with isotropic receiver characteristics. The measured data at the $n$th receiver is:

$$
x_{n}(t)=E_{s}\left(\mathbf{r}_{\mathbf{n}}, t\right)+n(t)
$$

where $r_{n}$ is the vector from the origin to the $n$th receiver location and $n(t)$ is spatially and temporally white Gaussian noise. The sampled field $x_{n}(t)$ is then processed, and the location of the objects are estimated with the techniques described in Sections 4 and 5 . 


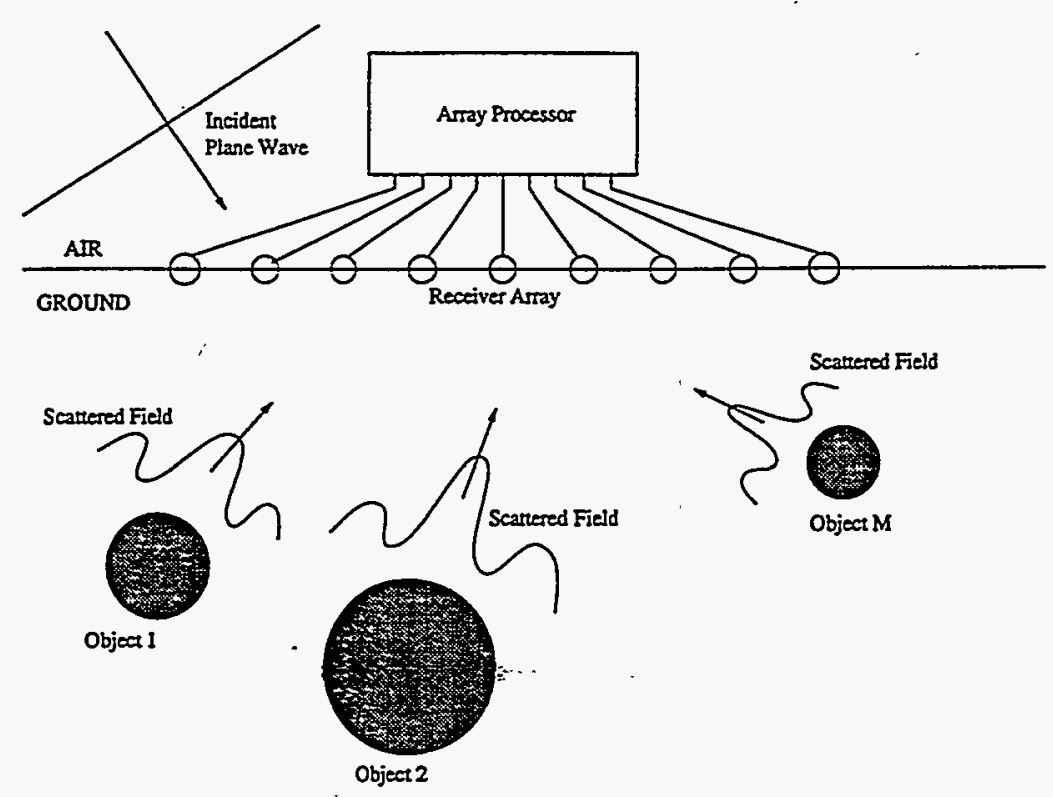

Figure 1: Problem Geometry

\section{High Resolution Array Processing and MUSIC}

The structure of the receivers in Fig. 1 coupled with the underlying problem of target detection suggests the use of array processing methods for localizing buried targets. In this report we consider techniques based on the MUSIC algorithm. As originally derived in [12], this method assumes that there are $M$ plane waves incident on a linear array of receiving elements so that the output of the $n$th sensor is:

$$
x_{n}(t)=\sum_{m=1}^{M} e^{j\left(\omega_{m} t-\mathbf{k}_{m}^{T} r_{n}\right)}+n(t)
$$

where $M$ is the total number of plane waves arriving at the sensors, $\omega_{m}$ is the frequency of the $m$ th signal, $k_{m}$ is the wave vector of the $m$ th signal and $r_{n}$ is the vector from the origin to the $n$th sensor location. By stacking such $x_{n}(t)$ 's, we form the following system:

$$
x(t)=A s(t)+n(t)
$$

where

$$
\begin{gathered}
\mathrm{A}=\left[\begin{array}{llll}
\mathrm{a}\left(\theta_{1}\right) & \mathrm{a}\left(\theta_{2}\right) & \ldots & \mathrm{a}\left(\theta_{M}\right)
\end{array}\right] \\
\mathbf{a}\left(\theta_{m}\right)=\left[\begin{array}{llll}
e^{j k_{m} \tau_{1} \cos \left(\theta_{m}\right)} & e^{j k_{m} \tau_{2} \cos \left(\theta_{m}\right)} & \ldots & e^{j k_{m} \tau_{N} \cos \left(\theta_{m}\right)}
\end{array}\right]^{T} \\
\mathbf{s}(t)=\left[\begin{array}{llll}
e^{j \omega_{1} t} & e^{j \omega_{2} t} & \ldots & e^{j \omega_{M} t}
\end{array}\right]^{T}
\end{gathered}
$$

Here $\mathrm{a}\left(\theta_{m}\right)$ is known as the direction vector, $\theta_{m}$ is the direction of arrival (DOA) of the $m$ th plane wave, $k_{m}=\left\|k_{m}\right\|$, and $r_{m}=\left\|\mathbf{r}_{m}\right\|$. The sensor outputs are sampled in time to form snapshots of the incoming signal. These snapshots are used to determine the statistics of $x(t)$. In particular, if $L$ time snapshots are taken, then the maximum likelihood estimate of the spatial autocovariance 
matrix $R$ is given by:

$$
\hat{\mathbf{R}}=\frac{1}{L} \sum_{l=1}^{L} \mathbf{x}\left(t_{l}\right) \mathbf{x}\left(t_{l}\right)^{H}
$$

where superscript $H$ denotes conjugate transpose.

The MUSIC algorithm is based on eigenspace decomposition of $\hat{\mathbf{R}}$. For an array of $N$ elements and $M$ incident signals $(N>M$ ), one can divide the $N$ eigenvalue/eigenvector pairs into $N-M$ noise eigenvectors corresponding to eigenvalues $\lambda_{M+1}=\ldots=\lambda_{N}=\sigma^{2}$ and $M$ signal eigenvectors corresponding to eigenvalues $\lambda_{1} \geq \ldots \geq \lambda_{M}>\sigma^{2}$ [9]. After performing an eigenvalue/eigenvector analysis, the autocovariance matrix is written as:

$$
\hat{\mathrm{R}}=\hat{U}_{s} \hat{\Lambda}_{s} \hat{U}_{s}^{H}+\hat{U}_{n}\left[\hat{\sigma}^{2} I\right] \hat{U}_{n}^{H}
$$

where $\hat{U}_{s}$ is the signal subspace matrix and contains the $M$ signal eigenvectors, and $\hat{U}_{n}{ }^{\prime}$ is the noise subspace matrix and contains $N-M$ noise eigenvectors of multiple noise eigenvalue $\hat{\sigma}^{2}$.

The basic idea behind MUSIC is that the columns of $\hat{U}_{s}$ span the same space as the columns of $A$ while the columns of $\hat{U}_{n}$ span an orthogonal subspace. Thus, a wave impinging on the array at an angle $\theta_{0}$, which is described by the vector $a\left(\theta_{0}\right)$, must be orthogonal to each of the columns in $\hat{U}_{n}$. In other words $\hat{U}_{n}^{H} \mathrm{a}\left(\theta_{0}\right)$ must, in theory, be equal to the zero vector. Thus, if one examines

$$
d_{M U S I C}(\theta)=\frac{a(\theta)^{H} \mathrm{a}(\theta)}{\mathrm{a}(\theta)^{H} \hat{\mathrm{U}}_{n} \hat{\mathrm{U}}_{n}^{H} \mathrm{a}(\theta)}=
$$

as a function of $\theta$, estimates of DOAs can be determined by the maxima of the curve.

The formulation of the array processing problem presented in this section implicitly assumes that the radiators are infinitely distant so that the scattered field has planar wavefronts at the sensor array. Thus, the elements of the direction vector $a(\theta)$ are complex exponentials indicative of plane wave signals. As shown in Figure 2, for the case of GPR the radiating sources are at a finite distance from the receivers resulting in non-planar wavefronts over the array. Additionally, these near-field problems require the determination of both the direction of the object relative, to some point on the array as well as the range of the target from the same point. The next two sections describe the proposed techniques to locate the targets in the light of this discussion.

\section{Matched Field Processing}

To overcome the problem of non-planar wavefronts and to produce estimates of both angle and range, MUSIC can be modified so that the direction vector is filled with the type of wavefront which is impinging on the array. For example, if one is concerned with the localization of point sources in the near field of the array, spherical MUSIC [4] can be employed to find both the DOAs and the distance of the object from the array. Spherical MUSIC uses $e^{-j k r_{m n}} / r_{m n}$ to fill in the direction vector, as opposed to plane wave MUSIC which uses $e^{-j k r_{n} \cos \left(\theta_{m}\right)}$, where $r_{m n}$ is the distance between the $n$th array receiver and the $m$ th source (object), $r_{n}$ is the distance between the origin and the $n$th receiver, $\theta_{m}$ is the DOA of $m$ th source, and $k$ is the wavenumber in the medium of propagation. If the source (or scatterer) is not a point source, then the direction vector should be filled with the expected scattered field from each object location. This approach is called the Matched Field Processing (MFP) in the underwater communication community [1]. As with spherical MUSIC, MFP parameterizes the problem in terms of range and angle variables and the 


\section{Incident planewave}
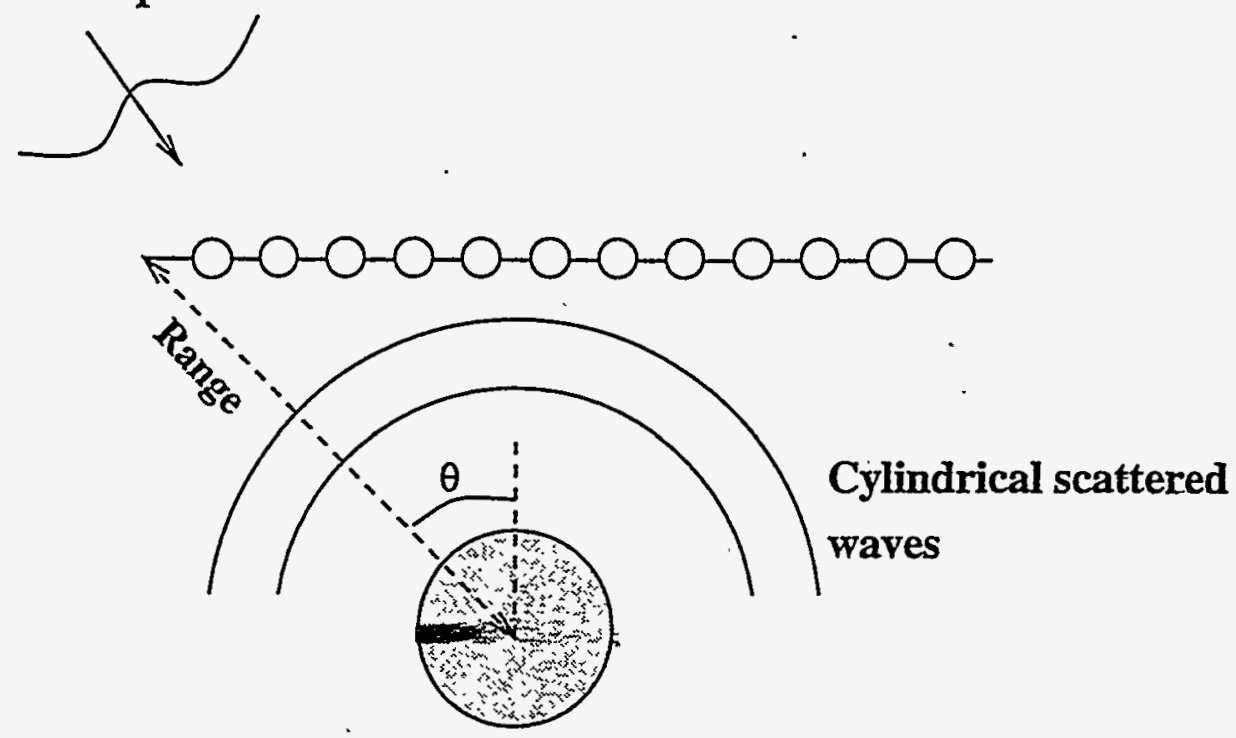

Figure 2: The Near Field MUSIC Problem

detection of $N$ targets is obtained using a $2 N$ dimensional search. Because such a search requires that the exact scattered field be calculated at each point of the parameter mesh, MFP is in general computationally intensive. In the event that the object to be detected is modeled as a simple shape, however, computing the exact scattered field can be relatively simple.

In this report, we consider the case where a single infinitely long cylindrical object is buried in a homogeneous, lossy background. To simplify the formulation, we ignore the air-earth interface and assume the receiver array is located in the same medium as the object. Following the derivation in [13], the $z$-component of a TM-polarized incident field is written as:

$$
E_{z}^{i}(\mathrm{r})=\sum_{l=-\infty}^{\infty} j^{-l} J_{n}\left(k_{0} r\right) e^{j l\left(\phi+\pi-\phi_{i n c}\right)}
$$

where $J_{l}($.$) is the Bessel function of order l$ representing cylindrical standing waves, $k_{0}$ is the wavenumber of the background, $\mathrm{r}=r e^{j \phi}$ is the location vector of the observation point and $\phi_{i n c}$ is the incidence angle of the plane wave ${ }^{1}$. The $z$-component of the scattered field outside the cylinder due to the incident wave is then given by:

$$
E_{z}^{s}(\mathrm{r})=\sum_{l=-\infty}^{\infty} j^{-l} t_{l} H_{l}^{(2)}\left(k_{0} r\right) e^{j l\left(\phi+\pi-\phi_{\text {inc }}\right)}
$$

where $H_{l}^{(2)}($.$) . is the Hankel function of the second kind of order l$ representing cylindrical outgoing waves ${ }^{2}$. The cöefficients $t_{l}$ are determined from the boundary conditions when $r=a$, where $a$ is the

\footnotetext{
${ }^{1}$ All analysis is in frequency domain, thus the $e^{j \omega t}$ time dependence will be dropped.

${ }^{2} \mathrm{Eq}$. (3) is true under the assumption that the center of the cylinder is located at the origin. In implementing the MFP MUSIC algorithm, coordinate translations are required to account for the fact that the position of the object is generally not at the origin of the global coordinate system. While tedious to describe, these translations do not significantly alter the computational load or functional form implied by eq. (3).
} 
radius of the cylinder. Forcing continuity of $z$-component of total electric field and $\phi$-component of total magnetic field strength, the coefficients $t_{l}$ are found as:

$$
t_{l}=\frac{J_{l}(k a) J_{l}^{\prime}\left(k_{0} a\right)-\sqrt{\epsilon_{r}} J_{l}^{\prime}(k a) J_{l}\left(k_{0} a\right)}{\sqrt{\epsilon_{r}} J_{l}^{\prime}(k a) H_{l}^{(2)}\left(k_{0} a\right)-J_{l}(k a) H_{l}^{(2)^{\prime}}\left(k_{0} a\right)}
$$

where primes indicate differentiation with respect to the argument. In practical implementations, the infinite sums in (1) and (2) are truncated at $l=\Lambda$. For all cases in this report $\Lambda=40$ provides an accurate representation of the fields.

Given the field model in eq. (3), the matched field processing. MUSIC algorithm proceeds as follows:

1. Perform $L$, single-frequency scattering experiments each producing a snapshot vector, $\mathbf{x}_{l}$, composed of the observed scattered fields over the receiver array.

2. Estimate the autocovariance matrix as $\hat{\mathrm{R}}=\frac{T}{L} \sum_{l=1}^{L} \mathrm{x}_{l} \mathrm{x}_{l}^{H}$.

3. Perform an eigenanalysis on $\hat{R}$ to find the noise-subspace matrix $\hat{U}_{n}$.

4. For each point on a predefined range and angle grid, use (4) to compute a direction vector, $\mathrm{a}(\theta, r)$, which represents the expected distribution of scattered field over the receiver array when an object is located at an angle $\theta$ and range $r$ off of the array. The vector $a(\theta, r)$ computed in this manner replaces $a(\theta)$ in (2).

5. Choose as the estimate of the target location that grid point with the largest $d_{M U S I C}$.

\section{Subarray Processing}

While matched field processing does solve many of the problems associated with the near field scatterers, even in the single cylinder case the computational burden is higher than that of plane wave MUSIC. As an alternate approach, we propose a strategy in which the full receiver array is partitioned into non-overlapping collection of sub-arrays such that the the wavefronts are in fact planar over each partition. The direction of arrival at each subarray, then, can be found using the traditional, plane wave based MUSIC algorithm, at a low computational cost, and the locations of the objects are determined by triangulation of direction of arrivals.

\section{Numerical Examples}

To demonstrate the potential use of high resolution array processing techniques in subsurface target detection applications, we considered the waste container detection problem where a single metallic cylinder ( $60 \mathrm{~cm}$ in diameter) is buried $120 \mathrm{~cm}$ below an array of 16 receivers with an aperture of $50 \mathrm{~cm}$. As shown in Fig. 3, two scenarios are of interest. In the first, the object is located directly beneath the center of the array while in the second case, the buried cylinder lies $50 \mathrm{~cm}$ to one side of the receivers. For each scenario, we examine the performance of MFP and the SAP algorithms under a variety of signal to noise (SNR) and soil conditions. Finally, we note that the operating frequency for all examples is $1 . \mathrm{GHz}$ and the incident plane wave used to probe the medium is directed normal to the surface.

In this work, the signal to noise ratio is defined as:

$$
S N R=10 \log _{10} \frac{E_{s}^{H} E_{s}}{N \sigma^{2}}
$$



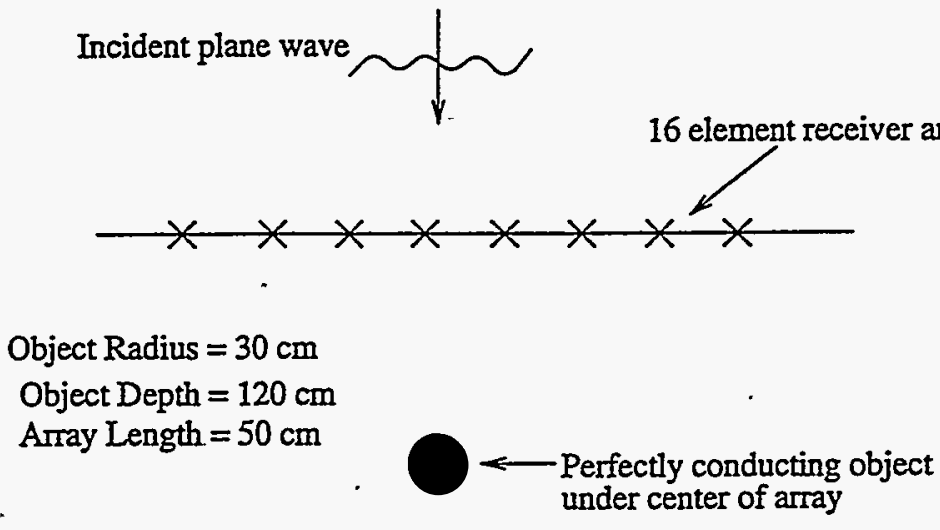

(a) Centered target case

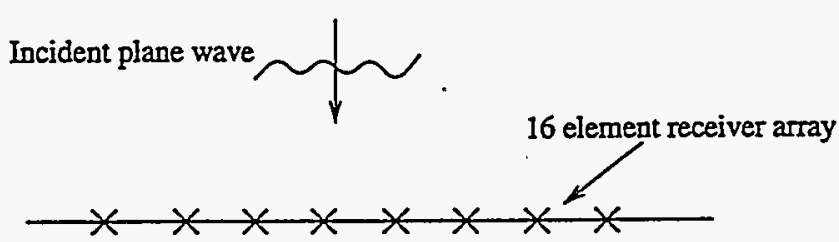

Object Radius $=30 \mathrm{~cm}$

Object Depth $=120 \mathrm{~cm}$

Horiz. distance from array $=50 \mathrm{~cm}$

Array Length $=50 \mathrm{~cm}$

Perfectly conducting object under center of array

(b) Off-center target case

Figure 3: Two detection geometries of interest in this work

where $\underline{E}_{s}$ is the scattered field sampled at the receivers, $N$ is the length of vector $\underline{E}_{s}$ and $\sigma^{2}$ is the noise variance. The estimation error is defined as:

$$
\operatorname{Error}^{2}=\frac{(\Delta x)^{2}+(\Delta y)^{2}}{a^{2}}
$$

where $\Delta x$ and $\Delta y$ are the difference between true center coordinates $\left(x_{0}, y_{0}\right)$ and estimated center coordinates $(\hat{x}, \hat{y})$ and $a$ is the radius of the target. Thus, (5) defines the error in terms of fractions of the object radii. In particular, errors less than 1 indicates that the estimated object center lies in the support of the object.

\subsection{Matched Field Processing Results}

Fig. 4 depicts the result of MFP MUSIC for the case in which the object is centered under the array and the SNR is $20 \mathrm{~dB}$. The background soil is $5-10 \%$ moist San Antonio clay loam [8]. As seen from this figure, the location of the object is identified clearly by a very sharp peak. For this example, the location of the target is exactly estimated, i.e. bearing angle of $90^{\circ}$ and range of 120 


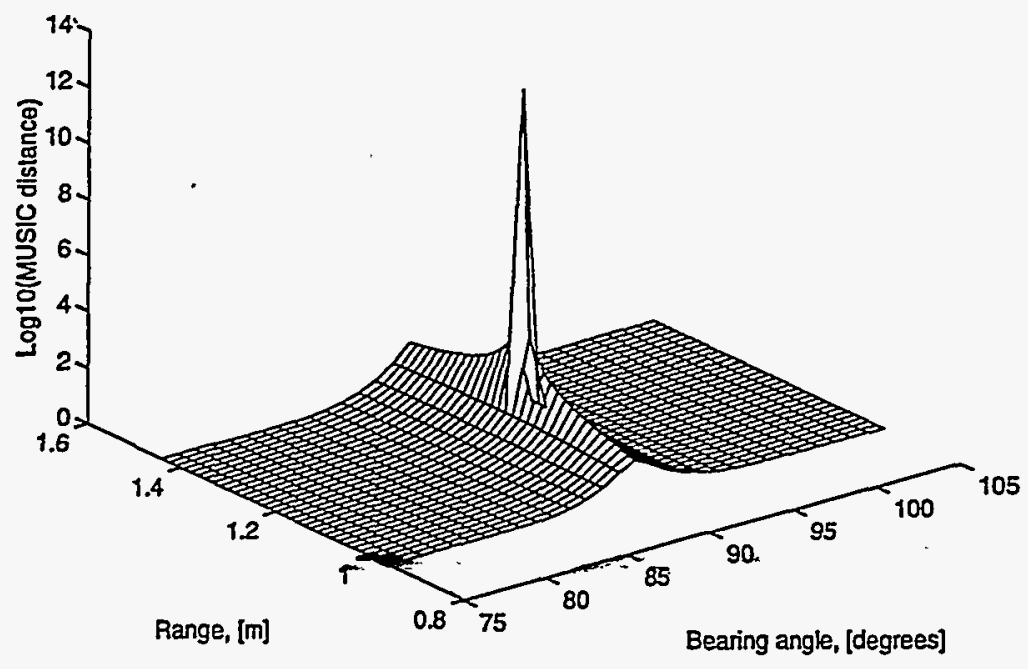

Figure 4: MFP MUSIC results for metallic object buried $120 \mathrm{~cm}$ below the center of the array. The SNR is $20 \mathrm{~dB}$ and the soil corresponds to \#3.

$\mathrm{cm}$. Our studies have shown that the size of the peak depends on the gridding of the $(r, \theta)$-space. If any grid point coincides with the true center of the target, a peak like in Fig. 4 is obtained. If the grid points are off the true center, the peak becomes smaller though stays clearly distinguishable.

MFP MUSIC was tested for a wide range of soil types with moisture contents up to $20 \%$. For this purpose, we varied the background permittivity and conductivity within the ranges given in [8] keeping the SNR at 20dB. Again, the estimation error for all background characteristics was virtually zero when a grid point coincides with the true object center, or the estimation error was bounded by the grid size. Finally, the noise performance of MFP MUSIC noise has proven to be quite strong. In all our simulations, the target locations were clearly distinguished for SNRs greater than $0 \mathrm{~dB}$.

\subsection{Subarray Processing Results}

Fig. 5(a) shows the results of the subarray processing algorithm for the centered target case when the electrical characteristics of the background is similar to $5-10 \%$ moist San Antonio clay loam and the SNR is $20 \mathrm{~dB}$. In Fig. 5(b), the SAP results for the off center case are displayed for the same SNR and soil conditions. In both cases the array is partitioned into 4 identical subarrays. The actual center of the object is marked with an $\mathrm{X}$ and object is superimposed to show the geometry. As seen from the figures, the lines extended at the DOAs of subarrays cross each other inside the object both for the centered as well as the off-centered target scenarios. Unlike MFP MUSIC, for the subarray processing algorithm, there is not one point identified as the center of the object because in general the triangulated DOAs. will not intersect at a single point. Rather, for $S$ subarrays there will be $(S-1)$ ! intersection points. Thus, in this report we take the estimate of the $x$ (resp. $y$ ) coordinate of the object center to be the average of the $x$ (resp. $y$ ) values taken over all $(S-1)$ ! intersection points. The error is then calculated using this number in eq. (3). Employing this procedure for the case in Fig. 5 , it is clear that the estimation error is only a fraction of the 


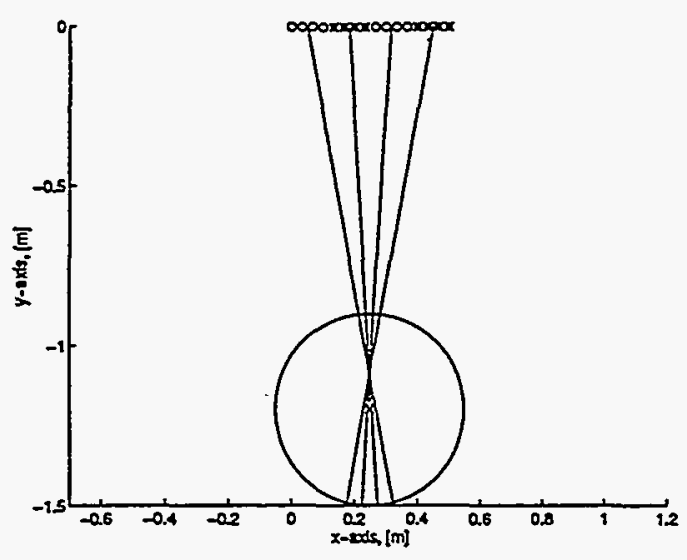

(a) Centered object case

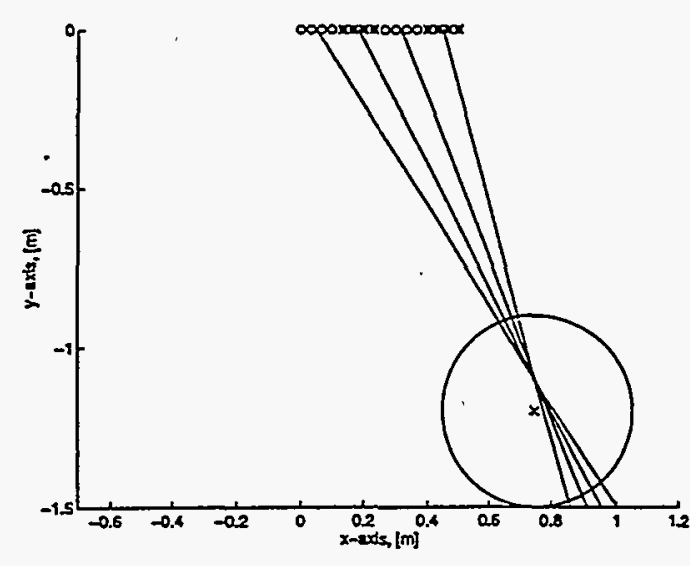

(b) Off-centered object case

Figure 5: Subarray processing results for (a) centered and (b) off-centered target scenarios. In both cases the SNR is $20 \mathrm{~dB}$ and the soil corresponds to \#3.

\begin{tabular}{|c|c|c|c|}
\hline & Type & Density $\left(\mathrm{g} / \mathrm{cm}^{3}\right)$ & Percent Moisture \\
\hline Soil \#1 & San Antonio Clay Loam & 1.6 & 5 \\
\hline Soil \#2 & Puerto Rican Clay Loam & 1.4 & 20 \\
\hline Soil \#3 & San Antonio Clay Loam & 1.4 & 20 \\
\hline
\end{tabular}

Table 1: Soils used to test noise performance for subarray processing

radius of the object. Therefore, the subarray processing estimates the center within the object's support.

The performance of the subarray processing scheme as a function of the soil conditions and signal to noise ratio is displayed in Fig. 6 again for the cases of the centered object (Fig 6(a)) and the off-centered target (Fig. 6(b)). Each of these figures displays the error as defined in (5) as a function of SNR for three soil types whose characteristics are given in Table 1. Each data point was obtained as the result of 50 Monte Carlo trials where 250 snapshots were used per trial to estimate the covariance matrix in (1). For all soils and for all SNRs greater than or equal to 0, the results of Fig. 6 indicate that the subarray algorithm is capable of identifying the target center to within the support of the true object (i.e. the error is less than 1). These simulations indicate that SAP represents an effective (and computationally efficient) means of localizing buried objects. It is important to note that the strong performance of the SAP strategy holds even in the cases of Soils \#2 and \#3 where the moisture content is as high as $20 \%$.

\section{Extensions to the Multi-Object Case}

In addition to the development and evaluation of array processing based algorithms for the case where a single object is buried in the receivers' field of view, we have begun work on extending these techniques to the multi-object case. This effort requires two components. First, because (4) is valid only for the case of a single scatterer, an alternate physical model must be developed to 


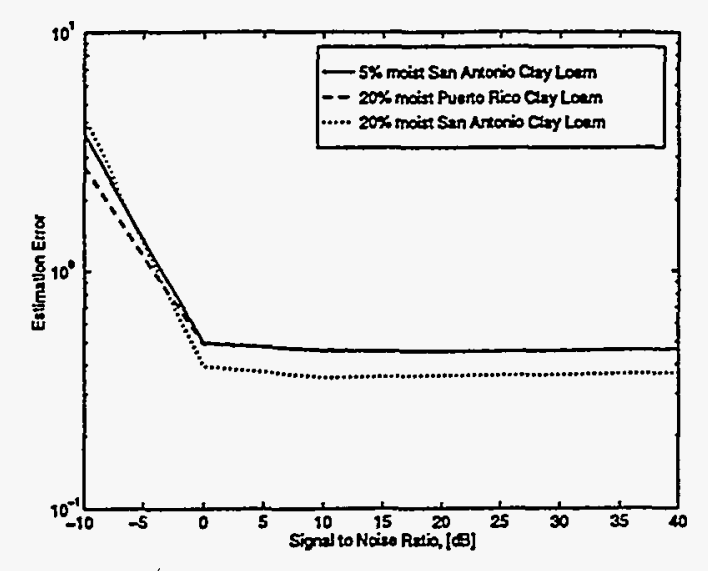

(a) Centered case

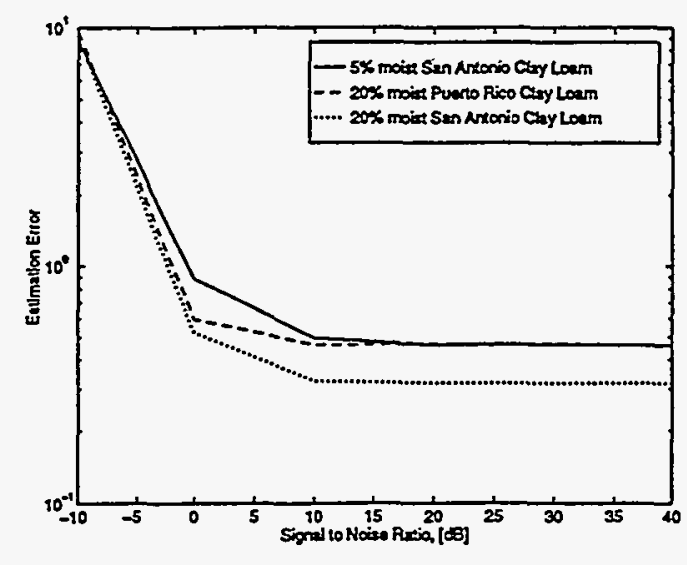

(b) Off centered case

Figure 6: Subarray processing results for (a) centered and (b) off-centered target scenarios as a function of signal to noise ratio and soil type. For all soil and for all SNRs greater than or equal to $0 \mathrm{~dB}$, the center of the object is estimated to within the support of the true structure.

describe the interaction of the incident fields with the buried objects. Second, processing schemes based on the MFP and SAP methods need to be developed which make use of this model. In this section, we discuss our recent efforts in both of these areas.

\subsection{T-Matrix Scattering Models}

When multiple objects exist in the region of interest, the scattering physics are, in general, significantly more complicated than the single scatterer model in (4). Typical numerical methods for solving this so-called forward scattering problem are based on a method of moments approach to the solution of a second-kind Fredholm integral equation $[7,10]$. Using this approach the area under investigation is pixelated at the rate of at least ten points per wavelength per spatial dimension. The dimensionality of the resulting linear system can be on the order of tens to hundreds of thousand for realistically interesting problem making the computational load unacceptably high.

To avoid this computational burden, we exploit the fact that we desire the characterization of a relatively small number of scattering objects which in general have relatively simple shapes. In this case, highly efficient $T$-matrix methods exist for computing the electric field at the receiver array. As an indication of the computations involved with this forward modeling approach, consider the case where there exists one object. Here, a $T$ matrix model is based on the formal expansion of the incident and scattered fields as

$$
\begin{aligned}
& E_{z}^{i}(r)=\sum_{n=-\infty}^{\infty} f_{n} \psi_{n}^{*}(r) \\
& E_{z}^{s}(r)=\sum_{n=-\infty}^{\infty} a_{n} \Re \psi_{n}(r)
\end{aligned}
$$

where $\psi_{n}(\mathbf{r})$ is the order $m$ Hankel function of the first kind and $\Re \psi_{m}(\mathbf{r})$ is the so-called regular (or non-singular) part of $\psi_{n}(r)$. As $E_{z}^{i}(r)$ is known, so too are the coefficients $a_{n}$. Hence the forward 
scattering problem is solved once the $f_{n}$ are determined. In [11], it is shown that use of (6a) and (6b) along with a multipole expansion of the Green's function result in a relationship between the $f_{n}$ and the $a_{n}$ of the form

$$
f_{n}=\sum_{m=-\infty}^{\infty} T_{n m} a_{n}
$$

where $T_{m n}$ is the $(m, n)$ th component of the doubly-infinite $T$ matrix. The advantage of this formulation arises from the fact that highly accurate solutions can be obtained for most practical scattering problems by truncating the sums in (6a) and (6b) at some $m=M$ where $M$ typically is less than 100. Moreover, the computational load remains at this level even in the case where more than one scatterer is present. Thus, the $T$ matrix in (7) can be inverted with orders of magnitude fewer computations than the large, moment method matrix.

A key component of the $T$ matrix method is determining $T_{m n}$ for different shaped objects. In generals, the elements of the $T$ matrix are expressed in terms of integrals over the boundary of the scatterer [11]. In this work, we have considered an implementation of the $T$ matrix method for circular objects where closed form expressions exist for these boundary integrals. Such a strategy provides a balance between generality and computational efficiency. Finally, we have implemented and verified a $T$ matrix code for the case where there are two objects in the region of interest. Extension of this model to the $N$-object case is reserved for future work, but should be relatively straightforward given the results in $[3,11]$.

\subsection{Multi-Object Algorithms and Preliminary Results}

To date we have considered a number of extensions of the SAP and MFP algorithms for locating a pair of objects. In the SAP case, each subarray produces direction of arrival estimates for two plane waves, one from each of the two objects. After triangulating the DOAs from each subarray and examining the intersections, we obtain estimates of two object locations. For the MFP approach, a maximization must be carried out in a four dimensional space corresponding to the range and angle coordinates for each of the two objects. To avoid the need to perform a costly exhaustive search, we obtain initial estimates of the object locations by first running the SAP algorithm. Subsequently, MFP is employed to "zoom in" on the true object centers. The optimization is carried out through a succession of line searches where we first maximize the MUSIC distance function for say the first range variable, $r_{1}$, holding the other range $\left(r_{2}\right)$ and both angle variables $\left(\theta_{1}\right.$ and $\left.\theta_{2}\right)$ at their previous estimates. Given this new value of $r_{1}$ the procedure is repeated this time maximizing with respect to $\theta_{1}$. The algorithm continues by cycling through the $r_{1}$ and $\theta_{1}$ variables until convergence. This approach then is repeated using $r_{2}$ and $\theta_{2}$.

In Figures 7, we display the SAP and MFP results for the case where two metallic, drumlike objects are buried beneath a 32 element array with a $75 \mathrm{~cm}$ aperture. One drum is located under the left edge of the array and the other is $1 \mathrm{~m}$ to the right of the first. The soil is a $5 \%$ moist San Antonio Clay loam, the SNR is $20 \mathrm{~dB}$, and the operating frequency is $1 \mathrm{GHz}$. It is evident from Figure 7(a) that under these conditions, the two-object SAP routine provides a highly accurate estimate of the first object's location. For the second cylinder, the estimate is in the correct vicinity but can clearly be improved. Using the SAP results as an initial guess as to the objects' true locations, Figure 7 (b) demonstrates improved localization after 2 iterations of the matched field processing algorithm described in the previous paragraph ${ }^{3}$. Here we see that the second object's center is now quite close

\footnotetext{
${ }^{3}$ Note that the estimate obtained after the second iteration for object \#1 is essentially identical to the result after the first iteration
} 


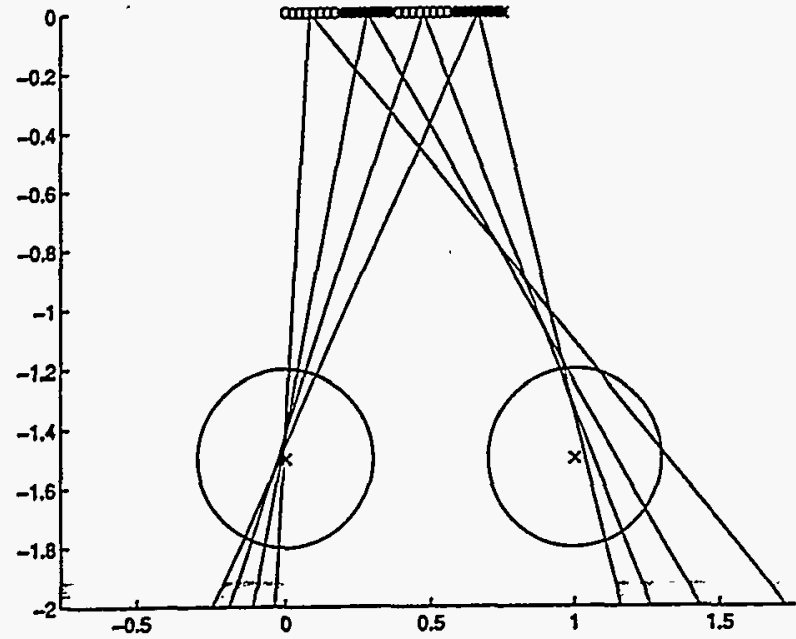

(a) Subarray processing results for two object experiment

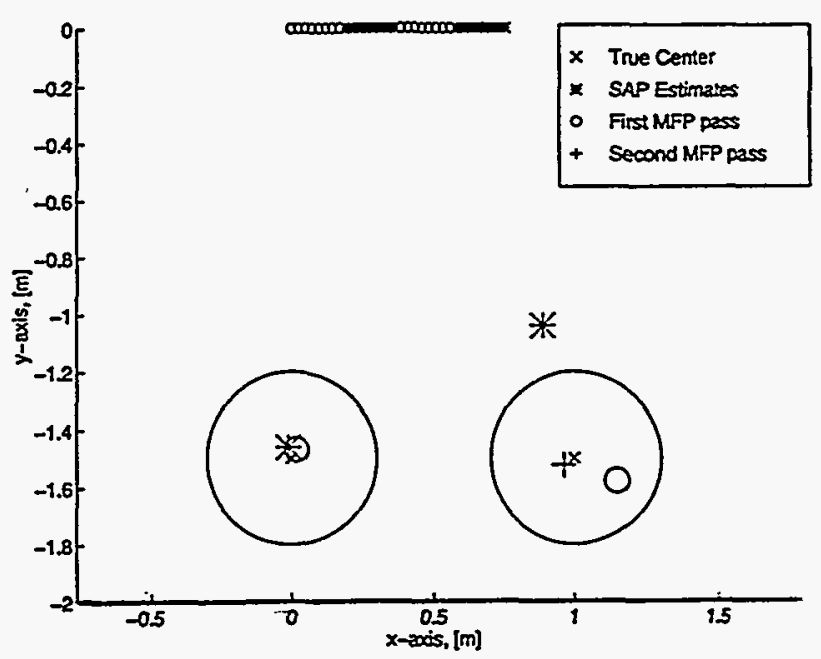

(b) Matched field processing results for two object experiment

Figure 7: Localization results for two-object scenario

to the true position, while the first object's position is improved slightly relative to the already accurate SAP result. For this problem, the MFP procedure was carried out by optimizing first with respect to $\left(r_{2}, \theta_{2}\right)$ and then over $\left(r_{1}, \theta_{1}\right)$. Future research efforts will be devoted to exploring how best to cycle through each of the $N$ pairs of coordinates.

\section{Conclusions and Plans for Future Work.}

In this report, the use of high resolution array processing techniques in a ground penetrating radar type measurement configuration has been demonstrated with examples. We have concentrated on the localization of a single object of known structure buried in an infinite background whose electrical characteristics are also known and have indicated preliminary results for the two object case. Using both matched field processing and subarray processing techniques we obtained very favorable results even under low SNR and high soil moisture conditions using a realistic measurement scenario. Out initial work indicates that an array processing approach should retain its favorable performance characteristics in the multi-object case as well.

Future research efforts are required in a number of areas. First, the $T$-matrix model must be generalized to allow for the calculation of scattered field for an arbitrary number of scattering objects. Additionally, while still maintaining its low computational complexity, the $T$-matrix method can be extended to compute fields arising from non-circular objects such as ellipses in two dimensions and spheres and ellipsoids in 3D. Clearly, the ability to efficiently model these types of objects increases the utility of the array processing methods for the hazardous waste removal applications.

Significant work also remains in extending the array processing algorithms. It is the case that the MFP distance surface has a number of local maxima surrounding the true maximum (which itself is close to the true object locations.) Thus, there is a need to devise computationally efficient methods which avoid convergence to a suboptimal estimate of the scatterers' locations. We anticipate that further work on the SAP-then-MFP method will be of use. Extensions of MFP 
(and SAP) to process data from multiple frequencies and multiple incident angles will be helpful in this regard. Additionally, the assumptions underlying the algorithms discussed in this report should be lifted. For example, it would be best if one did not need to specify a priori the number of objects buried beneath the array. A minimum description length type of criterion could be used to solve this problem. Similarly, rather than pre-defining the target properties (size, shape, electrical characteristics, etc.), a more robust procedure would involve only the specification of an object library. Given the data, the algorithms would determine which objects from the library are present.

Finally, extensive performance analysis remains to be done. As the array processing algorithms discussed here are statistical in nature, error variance and Cramer-Rao bounds can be computed for these methods. Such analysis can form the basis for understanding issues such as the sensitivity of the algorithms to the assumed soil conditions and the development of methods to estimate the soil electrical properties on line. Statistical performance indicators can be used to define the detectability and distinguishability of objects. Such information would be of use in building and searching the target Iibrary discussed in the previous paragraph, in determining the optimal frequencies for locating particular objects, etc.

\section{References}

[1] A. B. Baggeroerr, W. A. Kupperman, and Henrik Schmidt. Matched field processing: Source localization in correlated noise as an optimum parameter estimation problem. J. Acousti. Soc. $A m .$, 83(2):571-587, February 1998.

[2] Weng Cho Chew. Waves and Fields in Inhomogeneous Media. Van Nostrand Reinhold, New York, 1990.

[3] Weng Cho Chew, James A. Friedrich, and Robert Geiger. A multiple scattering solution for effective permittivity of a sphere mixture. IEEE Trans. Geoscience and Remote Sensing, 28(2):207-214, March 1990.

[4] J Choi and Y. Kim. Spherical beamforming and MUSIC methods for the estimation of location and strengths of spherical sources. Mechanical Systems and Signal Processing, pages 569-588, September 1995.

[5] A. J. Devaney. Geophysical diffraction tomography. IEEE Trans. on Geoscience and Remote Sensing, GE-22(1):3-13, January 1984.

[6] A. J. Devaney and G. A. Tsihrintzis. Maximum likelihood estimation of object location in diffraction tomography. IEEE Trans. ASSP, 39(3):672-682, March 1991.

[7] T. M. Habashy and R. Mittra. On some inverse methods in electromagnetics. Journal of Electromagnetic Waves and Applications, 1(1):25-58, 1987.

[8] Jackie E. Hipp. Soil electromagnetic parameters as functions of frequency, soil density, and soil moisture. Proceedings of the IEEE, 62(1):98-103, 1974.

[9] H.Krim and M.Viberg. Sensor array signal processing: Two decades later. Technical Report CICS-P-448, MIT Center for Intelligent Control Systems, Cambridge, Mass., January 1995. 
[10] Eric L. Miller and Alan S. Willsky. Wavelet-based methods for the nonlinear inverse scattering problem using the Extended Born Approximation. Radio Science, 31(1):51-67, JanuaryFebruary 1996.

[11] Bo Peterson and Staffan Strom. Matrix formulation of acoustic scattering from an arbitrary number of scatterers. J. Acoust. Soc. Am., 56(3):771-780, September 1974.

[12] Ralph O. Schmidt. Multiple emitter location and signal parameter estimation. IEEE Trans. Antennas and Propagation, AP-34(3):276-280, March 1986.

[13] Y. Wang. Theory and Applications of Scattering and Inverse Scattering Problems. PhD thesis, University of Ilinois at Urbana-Champaign, 1991. 


\title{
Chapter IVa
}

\section{Laboratory Soil Characterization}

\author{
WILLIAM H. WEEDON AND WEI SHI \\ Center for Electromagnetics Research \\ DEPARTMENT OF ELECTRICAL AND COMPUTER ENGINEERING \\ Northeastern UnIVersity, Boston, MA 02115
}

Final REPort on Grant DE-FC07-95ID13395

May 14, 1996 


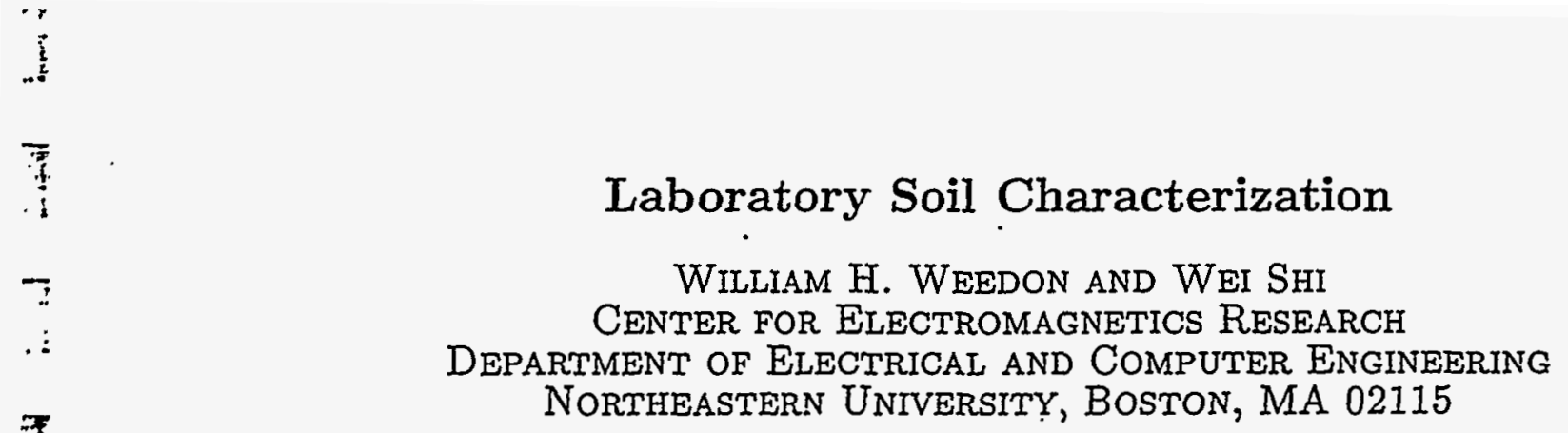

1

\begin{abstract}
A nondestructive coaxial sensor was investigated as a means of determining the frequencydependent permittivity of soils for modeling ground-penetrating radar. Accurate soil parameter extraction depends on both an internal sensor calibration and an external calibration that includes interactions with the test medium. Our internal calibration using a shorting plate and two resonant cavities has been verified to be accurate up to and above 1=5. GHE.: The external calibration based on quasi-static theory is good up to about 500 $\mathrm{MHz}$, but breaks down above this cutoff. Accurate soil measurements are now possible up to $500 \mathrm{MHz}$, but a new a new external calibration procedure needs to be developed to perform characterization above $500 \mathrm{MHz}$, particularly for high dielectric constant media. Our low frequency measurement cutoff is limited by the network analyzer's low frequency cutoff of $50 \mathrm{MHz}$.
\end{abstract}

\title{
1. Introduction
}

A laboratory soil characterization facility has been developed at Northeastern University to measure frequency-dependent soil permittivity and conductivity values using an open-ended coaxial probe [1] and a HP8510 vector network analyzer. This system is currently capable of extracting accurate soil parameters in the frequency range $50 \mathrm{MHz}$ to $500 \mathrm{MHz}$. A diagram of the sensor is shown in Figure 1. A unique feature of this probe is the large $32.5 \mathrm{~mm}$ diameter aperture in the outer conductor, allowing for averaging of the effects of large-grain inhomogeneities.

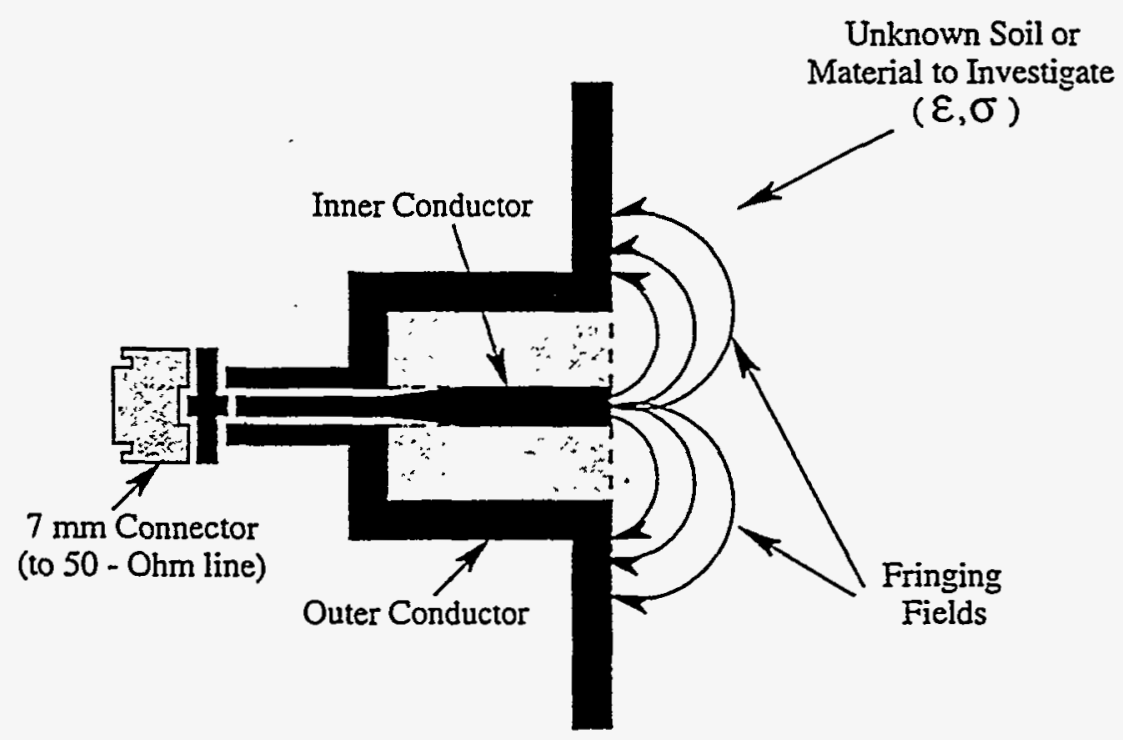

Figure 1: Coaxial near-field sensor used to infer dielectric constant and conductivity of soils. 
Accurate estimates of frequency-dependent permittivity and conductivity of test samples depend on calibration methods to remove systematic measurement errors. Our calibration procedure consists of two separate and distinct steps: an internal calibration procedure to remove losses and resonances inside the network analyzer, transmission lines, and the measurement cell itself; and an external calibration to account for the external capacitance and radiation conductance of the sensor. Custom calibration software was developed and used for both the internal and external calibrations.

A standard 3-term calibration model was used for the internal sensor calibration. The model parameters were determined by using test references consisting of a shorting plate and two cavities of different dimensions $[1,2]$. For the external calibration, the external fringing capacitance and radiation conductance of the probe were determined using a quasi-static analysis [3]. The quasi-static procedure is known to be valid for samples with relatively low dielectric constant and at frequencies such that the aperture dimensions are small compared to the wavelength in the test medium.

In order to test our internal calibration, the open-air response of the probe was measured. The measured admittance values show excellent agreement with quasi-static predictions up to $500 \mathrm{MHz}$. This valuable check is allowable since the open sensor response was not used as one of the calibration references. The open-air measurement may also be used to determine the external calibration parameters consisting of the frequency-dependent capacitance and the radiation conductance of the probe.

A non-linear parameter extraction algorithm was used to de-embed the frequencydependent permittivity and conductivity of the test samples from measured reflectivity data. To further verify the accuracy of the sensor, particularly for high dielectric media, both methanol and water were measured and compared to published Cole-Cole [4] and Klein-Swift models [5]. The results for these high-dielectric media compare favorably with the models for frequencies below $500 \mathrm{MHz}$, but diverged for frequencies above $500 \mathrm{MHz}$. The reason is that the quasi-static external calibration model that is typically used breaks down at around $500 \mathrm{MHz}$ for methanol and water. In order to obtain accurate parameter estimates above $500 \mathrm{MHz}$, particularly for high dielectric media, a new calibration model will need to be developed. A study of the variation of the electrical properties of the INEL soil with density and moisture is in-progress.

\section{Internal Sensor Calibration}

An internal sensor calibration procedure was used to remove the frequency-dependent response of the coaxial probe, network analyzer, and transmission line. A standard 3-term calibration procedure was used, which includes multiple resonances. The three calibration references used were a shorting plate and two resonant cavities with two different physical dimensions. Essentially, this internal calibration procedure allows us to reference the complex reflection coefficient measured by the network analyzer to the tip of the nondestructive sensor.

The complex reflection côfficient $\Gamma_{m}$ measured by the network analyzer may be related to the true reflection $\Gamma$ coefficient at the tip of the sensor as

$$
\Gamma_{m i}=S_{11}+\frac{\Gamma_{i} S_{12} S_{21}}{1-\Gamma_{i} S_{22}} \quad i=1,2,3 .
$$

where $i=1,2,3$ represent three independent calibration measurements. The calibration model parameters $S_{11}, S_{12}, S_{21}, S_{22}$, with $S_{21}=S_{12}$ are to be determined. In many applications, the product $S_{11} S_{22}$ is second-order and may be ignored, simplifying the determination of the S-parameters from the calibration measurements. This product cannot 
be ignored here, since the sensor contains a transition region where the ininer conductor flares. Hence, $S_{22}$ is not small.

Fortunately, the S-parameters may be determined exactly from the set off three nonlinear equations (1) as follows $[2,6]$ :

$$
\begin{gathered}
f=\frac{\left(\Gamma_{m 1}-\Gamma_{m 2}\right)\left(\Gamma_{1}-\Gamma_{3}\right)}{\left(\Gamma_{m 1}-\Gamma_{m 3}\right)\left(\Gamma_{1}-\Gamma_{2}\right)} \\
S_{22}=\frac{(1-f)}{\Gamma_{3}-f \Gamma_{2}}, \\
S_{21}=S_{12}=\sqrt{\frac{\left(\Gamma_{m 1}-\Gamma_{m 2}\right)\left(1-\Gamma_{1} S_{22}\right)\left(1-\Gamma_{2} S_{22}\right)}{\left(\Gamma_{1}-\Gamma_{2}\right)}} \\
S_{11}=\Gamma_{m 1}-\frac{S_{21} S_{12} \Gamma_{1}}{1-\Gamma_{1} S_{22}} .
\end{gathered}
$$

Once the S-parameters are determined, Equation (1) may be used to solve for the reflection coefficient $\Gamma$ at the tip of the probe from the reflection coefficient $\Gamma_{m}$ measured by the network analyzer.

\section{External Sensor Calibration}

The external procedure is used to relate the complex reflection coefficient at the tip of the sensor to the unknown medium parameters. A circuit model is usually chosen where the unknown medium that the sensor is in contact with is replaced by an equivalent complex admittance $Y(\omega)$. The admittance model typically used $[1,6]$ for this purpose is

$$
Y(\omega)=j \omega C_{f}+j \omega \widetilde{\epsilon}_{r} C_{0}\left(\omega, \widetilde{\epsilon}_{r}\right)+\tilde{\epsilon}_{r}^{2: 5} G_{0}(\omega)
$$

where $\tilde{\epsilon}_{r} \doteq \epsilon_{r}-j \sigma / \omega \epsilon_{0}$ is the complex relative permittivity of the unknown medium, $C_{f}$ is a fringing capacitance independent of the medium, $C_{0}\left(\omega, \epsilon_{r}\right)$ is the fringing capacitance due to the sample, $G_{0}(\omega)$ is the radiation conductance. The external calibration procedure consists of the determination of $C_{f}, C_{0}\left(\omega, \epsilon_{r}\right)$, and $G_{0}(\omega)$. The admittance $Y(\omega)$ may be obtained from the measurement $\Gamma$ as $Y(\omega)=(1-\Gamma) /(1+\Gamma) Z_{0}$, where $Z_{0}=50$ Ohms, and the nonlinear Equation (6) solved for the complex permittivity $\widetilde{\epsilon}_{r}$.

A quasi-static procedure [3] was investigated as a means of determining $C_{0}\left(\omega, \epsilon_{r}\right)$ and $G_{0}(\omega)$. The fringing capacitance $C_{f}$ is small and usually neglected in a quasi-static analysis.

The quasi-static procedure involves solution of the two electrostatic integrals

$$
I_{1}=\int_{a}^{b} d \rho \int_{a}^{b} d \rho^{\prime} \int_{0}^{\pi} d \phi \frac{\cos \phi}{\sqrt{\rho^{2}+\rho^{\prime 2}-2 \rho \rho^{\prime} \cos \phi}}
$$

and

$$
I_{3}=\int_{a}^{b} d \rho \int_{a}^{b} d \rho^{\prime} \int_{0}^{\pi} d \phi \cos \phi \sqrt{\rho^{2}+\rho^{\prime 2}-2 \rho \rho^{\prime} \cos \phi}
$$

Then the sensor capacitance in the test medium is given as

$$
C_{0}\left(\omega, \epsilon_{\tau}\right)=a_{1}+a_{2} \tilde{\epsilon}_{r} f_{\mathrm{MHz}}^{2} \quad \mathrm{pF}
$$


where

$$
a_{1}=\frac{\dot{2} \epsilon_{0} I_{1}}{\ln (b / a)^{2}}
$$

and

$$
a_{2}=-\frac{(2 \pi)^{2} \mu_{0} I_{3}}{\epsilon_{0}^{2} \ln (b / a)^{2}}
$$

The resulting expression for $C_{0}$ was found to be with $a_{1}=.2458$ and $a_{2} / a_{1}=1.78 \times 10^{-8}$ and $f_{\mathrm{MHz}}$ the frequency in $\mathrm{MHz}$. The value of $C_{f}$ was set to zero for our work. The radiation conductance determined from the quasi-static procedure is given as

$$
G_{01}(\omega)=\frac{\omega^{4} \pi \mu_{0}^{1.5} \epsilon_{0}^{2.5}}{12}\left[\frac{b^{2}-a^{2}}{\ln (b / a)^{2}}\right]^{2}
$$

Evaluation of (12) gives $G_{01}=3.527 \times 10^{-33}$ for our probe.

A slightly different expression for radiation conductance was dérived by Marcuvitz [7] using a variational formulation. Marcuvitz' expression may be written as

$$
G_{02}(\omega)=\frac{\omega^{4} \pi \mu_{0}^{1.5} \epsilon_{0}^{2.5}}{10}\left[\frac{b^{2}-a^{2}}{\ln (b / a)}\right]^{2}
$$

Note that Equations (12) and (13) differ only in the 1/12 and 1/10 factor and the square of the $\log (b / a)$ factor in the denominator.

\section{Results for Known Reference Media}

To verify the accuracy of the internal calibration procedure, reference media including air, methanol, and water were used. First, the open-sensor response of the probe was measured. The radiation conductance was determined from $G_{0 m}=\Re e\left(Y_{l m}\right)$, where $Y_{l m}=$ $\frac{1-\Gamma_{m}}{\left(1+\Gamma_{m}\right) Z_{0}}$, and the capacitance was determined from $C_{0 m}=-\Im m\left(Y_{l m}\right) / \omega$. Figures 2 and 3 show the measured radiation conductance and capacitance of the probe, as compared to theory. The agreement is excellent out to $1.5 \mathrm{GHz}$. This supports the assertion that the internal calibration procedure is working correctly, and that the measurements are accurate, at least in air.

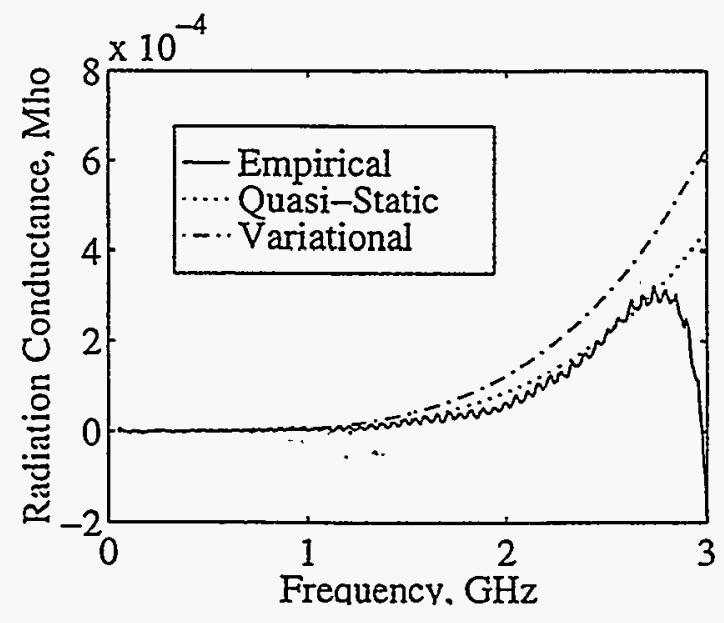

Figure 2: Radiation conductance of coaxial sensor derived empiricaly from open-sensor in air and compared to values from quasic-static [3] and variational [7] analysis procedures. 


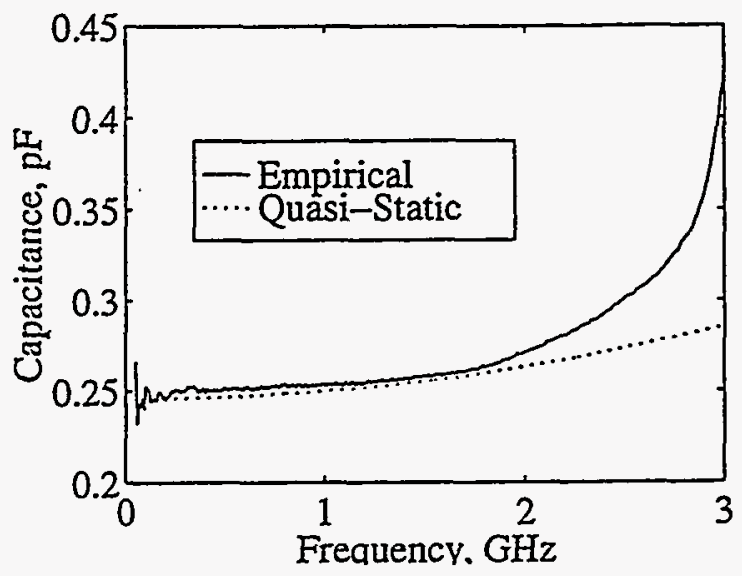

Figure 3: Capacitance of coaxial sensor derived empirically from open-sensor. in air and compared to quasi-static calculation [3].

Next, a similar procedure was applied to determine the radiation conductance and sensor capacitance from water and methanol. If Equation (6) is valid, then the radiation conductance $G_{0}(\omega)$ should be independent of the test medium used. Similarly, if the quasistatic procedure used to determine $C_{0}\left(\omega, \tilde{\epsilon}_{r}\right)$ is valid, the coefficients $a_{1}$ and $a_{2}$ derived using the quasi-static procedure should remain constant as the test medium is changed.

In order to characterize the sensor using methanol and water, a Cole-Cole model [4] was used to determine the accepted permittivity and conductivity for methanol, and the Klein-Swift model [5] was used to determine the values for water. A resistivity of 30 $\mathrm{Ohm}-\mathrm{m}$ and temperature of $20^{\circ} \mathrm{C}$ was assumed for the water.

Figures 4 and 5show the comparison of the radiation conductance and capacitance of the coaxial sensor derived empirically in methanol and water as compared to the quasistatic calculation, using the Cole-Cole [4] and Klein-Swift [5] models for complex permittivity. Note that the three curves agree well up to about $500 \mathrm{MHz}$, but that there are significant differences above $500 \mathrm{MHz}$. The implication is that the external capacitance model given by Equation (12) is not valid for high dielectric constant media, like methanol and water, above $500 \mathrm{MHz}$.

\section{Conclusions and Directions for Future Work}

A soil characterization laboratory has been developed for measuring the frequencydependent dielectric constant and conductivity of soil samples in the $50 \mathrm{MHz}$ to $500 \mathrm{MHz}$ region. Both internal and exteral calibration techniques were developed to remove systematic measurement errors associated with the sensor and data collection system. The internal calibration was verified to be extremely accurate up to $1 \mathrm{GHz}$, independent of the test sample. The external calibration technique breaks down near $500 \mathrm{MHz}$, particularly for media with high dielectric constant, due to limitations of the quasi-static theory. A new external calibration procedure needs to be developed in order to extend the sensor for high-dielectric constant media and frequencies $500 \mathrm{MHz}$ and above.

An obvious extension of this work is to incorporate the measured soil properties into electromagnetic modeling algorithms to compute detection depths for various targets (metallic vs. dielectric) in real soil environments. We are currently developing techniques 


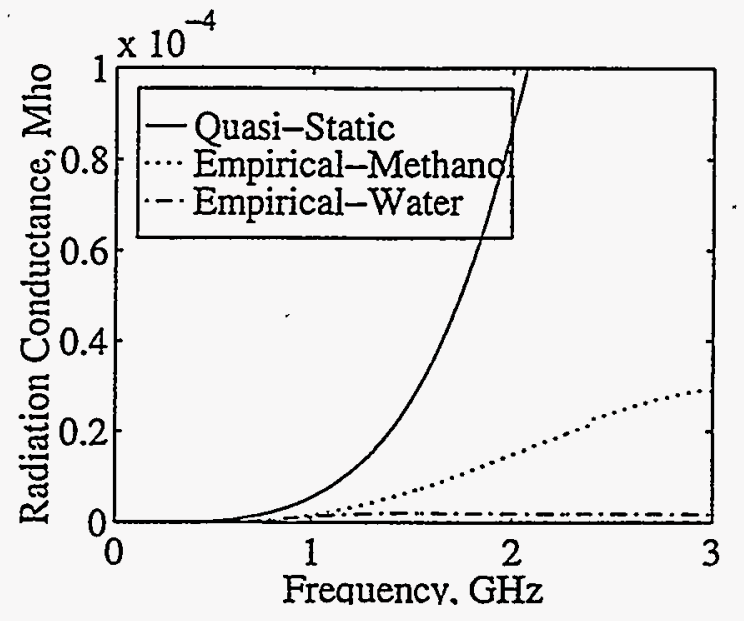

Figure 4: Radiation conductance coaxial sensor in methanol and water determined empirically and compared to quasi-static value.

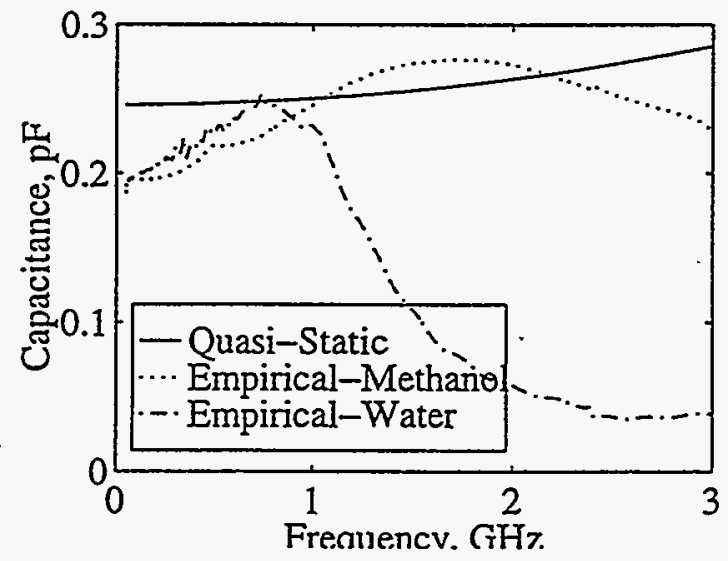

Figure 5: Capacitance of coaxial sensor in methanol and water derived empirically and compared to quasi-static value.

for incorporating these measured soil parameters into our computational models $[8,9,10$, 11]. In addition, the measurement equipment could easily be ruggedized and taken into the field to perform on-site soil measurements. This would allow field engineers to select appropriate antennas and tune their equipment for the specific soil conditions.

\section{References}

[1] G. P. Otto and W. C. Chew: "Improved calibration of a large open-ended coaxial probe for dielectric measurements," IEEE Trans. on Instrum. Meas., vol. 40, no. 4, pp. 742-746, 1991.

[2] W. C. Chew, K. J. Olp, and G. P. Otto, "Design and calibration of a large broadband dielectric measurement cell," IEEE Trans. Geosci. Remote Sensing, vol. 29, no. 1, pp. 42-47, 1991.

[3] D. K. Misra, "A quasi-static analysis of open-ended coaxial lines," IEEE Trans. Microwave Theory Tech., vol. MTT-35, no. 10, pp. 925-928, 1987.

$$
\text { IVa-6 }
$$


[4] B. P. Jordan, R. J. Sheppard, and S. Szwarnowski, "The dielectric properties of fromamide, ethaneidol and methanol," J. Phys. D: Appl. Phys., vol. 11, pp. 695-701, 1978.

[5] L. A. Klein and C. T. Swift, "An improved model for the dielectric constant of sea water at microwave frequencies," IEEE Trans. Antennas Propagat., vol. AP-25, no. 1, pp. 104-111, 1977.

[6] T. P. Marsland and S. Evans, "Dielectric measurements with an open-ended coaxial probe," IEE Proc., vol. 134, pt. H, no. 4, pp. 341-349, 1987.

[7] N. Marcuvitz, Waveguide Handbook. New York: McGraw-Hill, 1950.

[8] W. H. Weedon and C. M. Rappaport, "A general method for FDTD modeling of wave propagation in arbitrary frequency-dispersive media," IEEE Trans. Antennas Propagat., 1996. Submitted for publication.

[9] W: H. Weedon and C. M. Rappaport, "A general method for the modeling of arbitrary frequency-dispers media using FDTD," in Progress in Electromag. Research Symposium, (Seattle, WA), July 1995.

[10] W. H. Weedon, C. M. Rappaport, and D. Silevitch, "Modeling and stability considerations for FDTD analysis of wave propagation in lossy dispersive soils," in SPIE AeroSense 96: Radar Sensor Technology, (Orlando, FL), April 8-12 1996. To appear in SPIE Proceedings Vol. 2747.

[11] C. M. Rappaport and W. H. Weedon, "Efficient modeling of electromagnetic characteristics of soil for FDTD ground penetrating radar simulation," in IEEE Antennas and Propagat. Soc. Int. Symposium, (Baltimore, MD), July 21-26 1996. 


\section{Chapter IVb}

\section{Dispersive Soil Propagation Modeling}

William H. Weedon, Carey M. Rappaport and Daniel Silevitch

CENTER FOR ELECTROMAGNETICS RESEARCH

DEPARTMENT OF ELECTRICAL AND COMPUTER ENGINEERING

NORTHEASTERN UNIVERSTTY, BOSTON, MA 02115

Final Report on Grant DE-FC07-95ID13395

May 14, 1996 


\title{
Dispersive Soil Propagation Modeling
}

\author{
William H. WeEDon, Carey M. Rappaport and Daniel Silevitch \\ CENTER For ELECTROMAgNeTiCS RESEARCH \\ DEPARTMENT OF ELECTRICAL AND COMPUTER ENGINEERING \\ NORTHEASTERN UNIVERSITY, BOSTON, MA 02115
}

\begin{abstract}
The finite-difference time-domain (FDTD) algorithm, when modified to include dispersion, is a convenient tool for analyzing wave propagation in lossy, dispersive soils. It has been shown recently that a convenient way to include the dispersion is through a spatial array of digital permittivity or conductivity filters, one filter at each space node in the finite-difference grid. We address here the problem of seleeting filter coefficients that result in accurate and stable FDTD implementations. We present a systematic procedure for determining the coefficients based on a nonlinear optimization procedure and stability test.
\end{abstract}

\section{Introduction}

The finite-difference time-domain (FDTD) method is a powerful and versatile computational tool, and is presently one of the most popular computational methods. For ground-penetrating radar, FDTD may be used to predict transmitted waveforms in lossy, dispersive soils, and to compute fields scattered off of known targets. This type of modeling is also important for the inverse scattering problem, in order to quantitatively deduce subsurface physical properties from radar measurements. Numerical modeling is useful for determining penetration depths in inhomogeneous environments, and detectability studies for targets of interest such as land mines and groundwater contaminants.

There are certain instances where dispersion is not important. One case occurs when the radar system is inherently narrowband, and the soil electric properties may be considered constant over the narrow radar bandwidth. Another example is when one is only interested in shallow objects, such as near-surface mines, or in digface environmental or archaeological applications. But in general, the soil dielectric permittivity $\epsilon(\omega)$ and conductivity $\sigma(\omega)$ must be considered frequency dependent.

The standard Yee FDTD algorithm does not take dispersion into account, and the FDTD time-stepping equations must be modified in order to include dispersive effects. Several approaches have been proposed for incorporating dispersion into FDTD. These methods can roughly be categorized as: 1) differential equation methods $[1,2] ; 2$ ) discrete convolution algorithms $[3,4,5]$; and 3 ) $Z$-transform algorithms $[6,7]$. Recently, a new formulation has been proposed by the authors $[8,9,10]$ that includes many of these algorithms as special cases [8].

Our new method is more general than the previous methods because it does not rely on Debye, Lorentz, or Drude models, but rather is based on spatial arrays of digital filters. Two algorithms have been proposed: one using a permittivity filter (denoted form-1), and the other a conductivity filter (form-2). The filter parameters may be determined directly from measurement data, leading to finite difference implementations without the introduction of additional discretization error [8]. 
We address the problem of determining an optimal set of filter coefficients based on frequency-dependent soil data measured in a laboratory or in the field with appropriate soil characterization facilities. In particular, a step-by-step procedure is presented for determining parameters that result in accurate; stable FDTD implementations. We demonstrate our method with data from the classic 1974 Hipp paper [11] for Puerto Rico clay loam tabulated as functions of soil density and moisture.

\section{Dispersive FDTD Algorithms and Stability Test}

Two new dispersive FDTD algorithms have recently been proposed [8]. The first, which we denote as a form-1 dispersive FDTD algorithm, is based on a permittivity filter implementation of the dispersion relation

$$
D(r, Z)=\Delta_{t} \epsilon(r, Z) E(r, Z),
$$

the other, denoted form-2, is based condivity filter

$$
J_{c}(r, t)=\Delta_{t} \sigma(r, Z) E(r, Z) .
$$

In the above, $D(r, Z)$ and $E(r, Z)$ are $Z$-transforms of the electric flux density and electric field, and $J_{c}(r, Z)$ is an induced source current due to dispersion. The one-sided (causal) $Z$-transform of a vector field $A^{l}(r)=A\left(r, t=l \Delta_{t}\right)$ is defined as

$$
A(r, Z)=\sum_{l=0}^{\infty} A^{l}(r) Z^{-l}
$$

We model the dispersive media using Padé representations for $\epsilon(r, Z)$ and $\sigma(r, Z)$. In the form-1 algorithm,

$$
\epsilon(r, Z)=\epsilon_{0} \frac{b_{0}(r)+b_{1}(r) Z^{-1}+b_{2}(r) Z^{-2}}{1+a_{1}(r) Z^{-1}+a_{2}(r) Z^{-2}}
$$

and

$$
\sigma(r, Z)=\frac{d_{0}(r)+d_{1}(r) Z^{-1}+d_{2}(r) Z^{-2}}{1+c_{1}(r) Z^{-1}+c_{2}(r) Z^{-2}}
$$

for the form-2 algorithm. In the form-2 algorithm, we also include a frequency-independent background permittivity $\epsilon_{T}(r)$. In principle, we may include an arbitrary number of terms in the numerator and denominator of Equations (3) and (4). We limit the equations to second order in both numerator and denominator in order to minimize memory requirements, since the number of terms dictates how many previous time values of the fields must be stored at every node.

Our dispersive FDTD algorithms may be written in Z-transform space

$$
Z F_{i+1 / 2}(Z) \epsilon_{i+1 / 2} E_{x, i \div 1 / 2}=\frac{-\Delta_{t}}{\Delta_{z}}\left[H_{y, i+1}{ }^{\prime}-H_{y, i}\right]-\Delta_{t} J_{s, x, i+1 / 2}
$$

and

$$
\left(1-Z^{-1}\right) H_{y, i}=\frac{-\Delta_{t}}{\mu_{0} \Delta_{z}}\left[E_{x, i+1 / 2}-E_{x, i-1 / 2}\right]
$$

$\mathrm{IVb}-2$ 
Equation (5) and (6) are discretized and $Z$-transformed versions of the Ampere and Faraday Laws. We have only the $E_{x}$ and $H_{y}$ terms in Equations (5) and (6), corresponding to one-dimensional (1-D) wave propagation. These equations are easily generalized to 2-D and 3-D.

The function $F(Z)$ in Equation (5) corresponds to the dispersion, and takes on different forms for various FDTD algorithms. For the standard nondispersive FDTD algorithm,

$$
F(Z)=1-Z^{-1}
$$

For the form-1 dispersive FDTD algorithm,

$$
F(Z)=\frac{\epsilon(Z)}{\epsilon_{0}}\left(1-Z^{-1}\right)
$$

and for the form-2 dispersive FDTD algorithm,

$$
F(Z)=\left(1-Z^{-1}\right)+\frac{\Delta_{t}^{2}}{2 \epsilon_{i+1 / 2}}\left(1+Z^{-1}\right) \sigma_{i+1 / 2}(Z) .
$$

Combining (5) and (6), we may solve for the $Z$-dependent transfer function relating the electric field $E_{x, i+1 / 2}$ to the independent source $J_{s, x, i+1 / 2}$. We find that the denominator polynomial of this transfer function is given by

$$
(Z-1) F(Z)=-4 r^{2} s^{2}
$$

where $r=\frac{\Delta_{t}}{\Delta_{z} \sqrt{\mu_{0} \epsilon}}$ is the Courant number and $s^{2}=\sin ^{2}\left(k_{x} \Delta_{x} / 2\right)+\sin ^{2}\left(k_{y} \Delta_{y} / 2\right)+$ $\sin ^{2}\left(k_{z} \Delta_{z} / 2\right)$ for a plane wave. In general, $0<s^{2}<n$, where $n$ is the number of space dimensions in the simulation.

A necessary condition for stability of the FDTD algorithm is that the roots of Equation (10) lie within the unit circle, since this this equation describes the roots of the linear transfer function mapping the independent input function $J_{s, x}$ to the output $E_{x}$. It is interesting to note that Equation (10) may also be obtained by applying the Von Neumann stability criterion [8]. Hence, for stability of the FDTD algorithms, we require that the roots $Z_{k}, k=1, \ldots, N_{k}$ all lie within the unit circle. For the second-order Padé representations given by Equations (3) and (4), there are four roots $\left(N_{k}=4\right)$.

In Equation (10), $s$ relates to the direction of wave propagation in the FDTD grid, and is considered a nuisance parameter, because its value cannot be specified, and must be allowed to vary within $0<s^{2}<n$. This makes it difficult to specify regions of stability for the dispersive FDTD parameters $\left(b_{0}, b_{1}, b_{2}, a_{1}, a_{2}\right)$ or $\left(d_{0}, d_{1}, d_{2}, c_{1}, c_{2}\right)$ in Equations (3) and (4). Instead we apply a stability test, whereby a root-locus technique is used to ensure that the roots $\left|Z_{k}\right|<1, k=1, \ldots, N_{k}$ remain within the unit circle as the nuisance parameter $s^{2}$ is varied from 0 to $n$.

\section{Nonlinear Optimization of Filter Parameters}

Wie now consider the selection of optimal parameters $\left(b_{0}, b_{1}, b_{2}, a_{1}, a_{2}\right)$ and $\left(d_{0}, d_{1}, d_{2}, c_{1}, c_{2}\right)$ for the form-1 and form-2 dispersive FDTD algorithms, respectively, that result in accurate and stable FDTD implementations. This is essentially a filter design problem, but is 
complicated by the fact that we must accurately match both the magnitude and phase of the filter as a function of frequency. Note that Equations (3) and (4) are complex-valued, since it is not possible to have a causal, stable infinite-impulse response (IIR) digital filter with zero phase. The filter coefficients $\left(b_{0}, b_{1}, b_{2}, a_{1}, a_{2}\right)$ and $\left(d_{0}, d_{1}, d_{2}, c_{1}, c_{2}\right)$ are real, however.

It is tempting to apply a nonlinear optimization directly to obtain a set of filter coefficient that match the measured permittivity and conductivity data with $\epsilon(Z)$ and $\sigma(Z)$ evaluated on the unit circle $Z=e^{j \omega \Delta_{t}}$. This is not a viable solution in practice, however, due to the high sensitivity of $\epsilon(Z)$ and $\sigma(Z)$ to the pole locations $\left(a_{1}, a_{2}\right.$ or $\left.c_{1}, c_{2}\right)$. Instead, we specify the pole locations by hand, and apply a nonlinear optimization procedure to solve for the numerator polynomials of $\epsilon(Z)$ or $\sigma(Z)$.

To determine $a_{1}, a_{2}$ or $c_{1}, c_{2}$, we use reasoning-to determine where in the complex plane the poles of $\epsilon(Z)$ and $\sigma(Z)$ should lie. First, we note that the denominator polynomial $1+a_{1} Z^{-1}+a_{2} Z^{-2}$ may be factored as $\left(1-p_{1} Z^{-1}\right)\left(1-p_{2} Z^{-1}\right)$, where $p_{1}, p_{2}$ are the pole locations. Then $a_{1}=-\left(p_{1}+p_{2}\right)$ and $a_{2}=p_{1} p_{2}$. Note that $a_{1}$ and $a_{2}$ are not independent. In order for $a_{1}$ and $a_{2}$ to be real-valued, $p_{1}, p_{2}$ can either be real and distinct, or complexconjugate pairs. If they are real, then the poles occur either at $\omega=0$ if positive or $\omega=\infty$ if negative. A pole at infinity would amplify numerical noise in a FDTD algorithm, and a pole at DC dọes not seem to give any advantage in terms of matching the measurement data. We choose the poles $p_{1}$ and $p_{2}$ to be complex-conjugate pairs. In addition, we choose the undamped resonance frequency $\omega_{\text {res }}=\angle p_{1} / \Delta_{t}$ to lie within the bandpass region of interest (note that at the undamped resonance frequency $\omega_{\text {res }}, Z=p_{1}=e^{j \omega}{ }_{\text {res }} \Delta_{t}$ ). Once the phase of $p_{1}$ is specified, we apply an educated guess to choose the magnitude of $p_{1}$ between 0 and 1. Then, $p_{2}=p_{1} *, a_{1}=-\left(p_{1}+p_{2}\right)$, and $a_{2}=p_{1} p_{2}$.

Next, we solve for the numerator polynomial coefficients $\left(b_{0}, b_{1}, b_{2}\right)$ or $\left(c_{0}, c_{1}, c_{2}\right)$ of Equation (3) or (4). These values may be determined readily by applying a nonlinear optimization strategy. Using a least-squares error criterion, the form-1 coefficients may be determined by minimizing the cost function

$$
X^{2}=\sum_{k=1}^{K} \frac{\left[\sigma_{k}+\omega_{k} \epsilon_{0} \mathcal{I} m\left\{\epsilon\left(e^{j \omega_{k} \Delta_{t}}\right)\right\}\right]^{2}}{K \sigma_{k}^{2}}+\sum_{n=1}^{N} \frac{\left[\epsilon_{n}-\mathcal{R} e\left\{\epsilon\left(e^{j \omega_{n} \Delta_{t}}\right)\right\}\right]^{2}}{N\left(\epsilon_{n}-\epsilon_{r}\right)^{2}} .
$$

Similarly, the form-2 filter parameters may be determined from

$$
X^{2}=\sum_{k=1}^{K} \frac{\left[\sigma_{k}-\mathcal{R} e\left\{\sigma\left(e^{j \omega_{k} \Delta_{t}}\right)\right\}\right]^{2}}{K \sigma_{k}^{2}}+\sum_{n=1}^{N} \frac{\left[\left(\epsilon_{n}-\epsilon_{r}\right)+\mathcal{I} m\left\{\sigma\left(e^{j \omega_{n} \Delta_{t}}\right)\right\} / \omega_{n} \epsilon_{0}\right]^{2}}{N\left(\epsilon_{n}-\epsilon_{r}\right)^{2}}
$$

Once the numerator coefficients are determined, $\sigma(Z)$ or $\epsilon(Z)$ should be checked visually to ensure that the optimization generated reasonable results. Then, the stability test described in the previous section is applied. If the algorithm is not stable, the pole position can be re-adjusted and the optimization process repeated. If the algorithm is stable, then the coefficients can be used directly, or the pole positions can be moved and the optimization process repeated to reduce the cost function and obtain a better match to the data.

Figures $1-3$ show the results of our optimization process applied to the Puerto Rico clay loam data [11] for a form-2 algorithm. Figure 1 shows the results for a dry soil density 
of $1.2 \mathrm{~g} / \mathrm{cc}$, Figure 2 for a density of $1.4 \mathrm{~g} / \mathrm{cc}$, and Figure 3 for a density of $1.6 \mathrm{~g} / \mathrm{cc}$. In each figure, results are shown for various soil moisture percentages of the soil dry weight, ranging from $2.5 \%$ to $20 \%$. The optimization procedure generated reasonable results for all of the density/moisture values. The accuracy appears to be limited by the second-order model chosen for $\sigma(Z)$ in the form-2 dispersive FDTD algorithm. The model parameters are summarized in Table 1.

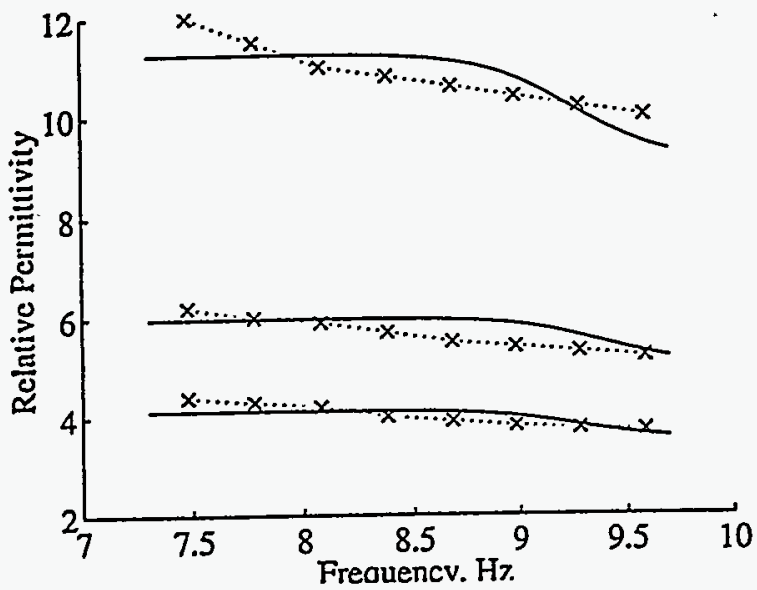

(a)

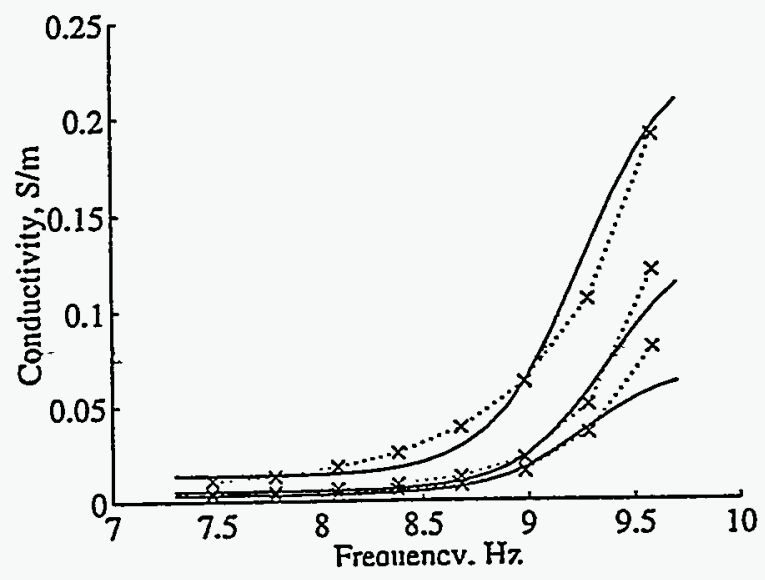

(b)

Figure 1. (a) Permittivity and (b) conductivity data versus frequency for $1.2 \mathrm{~g} / \mathrm{cc}$ dry density of Puerto Rico clay loam. Solid curves represent second-order Padé model, crosses represent measured data (Hipp, 1974). Various curves represent different percentages of moisture by dry soil weight.

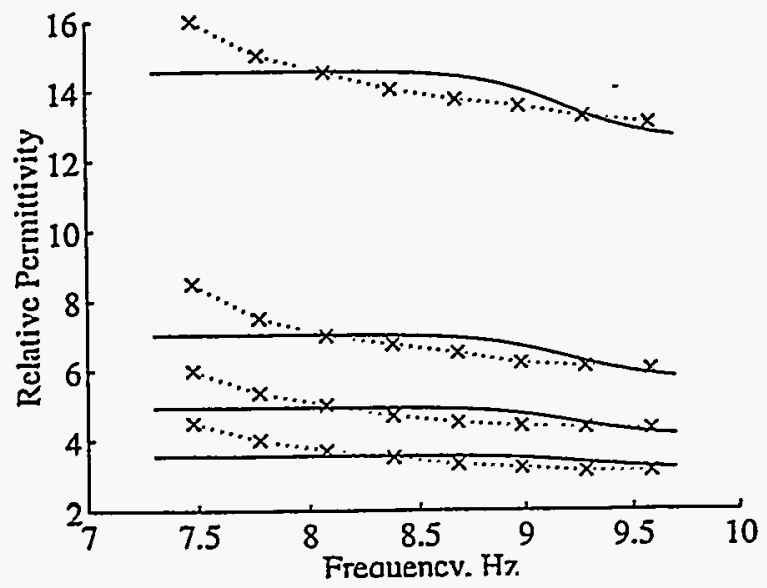

(a)

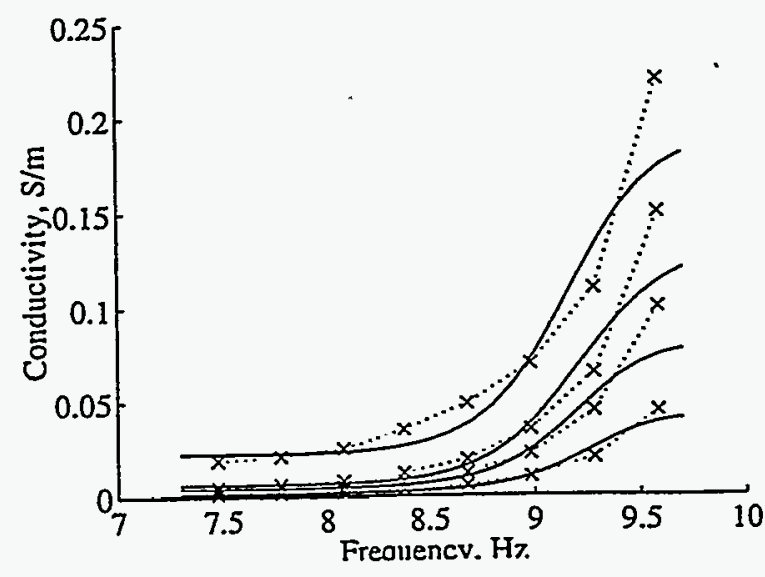

(b)

Figure 2. (a) Permittivity and (b) conductivity data versus frequency for $1.4 \mathrm{~g} / \mathrm{cc}$ dry density of Puerto Rico clay loam. See Figure 1. 


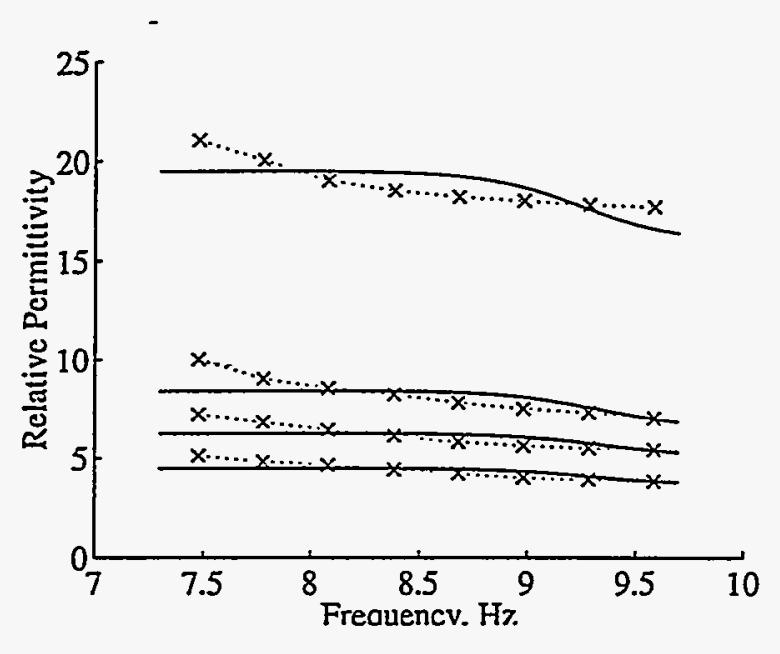

(2).

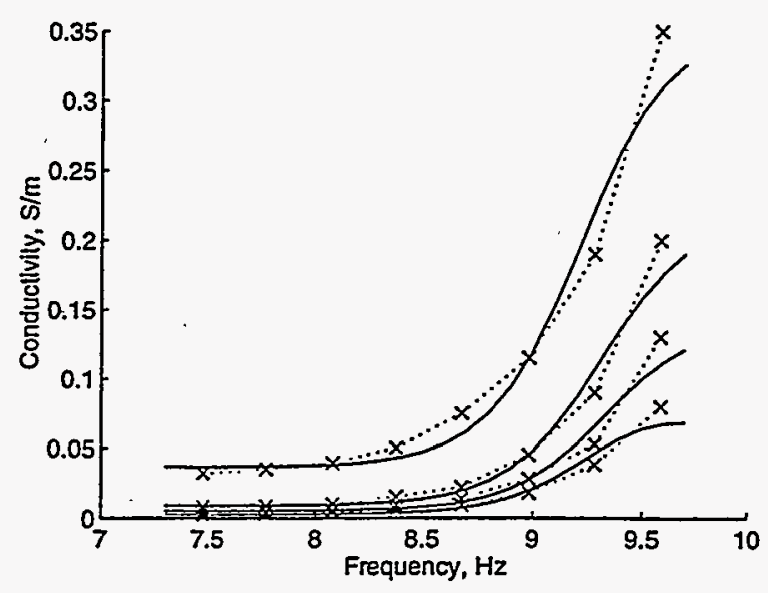

(b).

Figure 3. (a) Permittivity and (b) conductivity data versus frequency for $1.6 \mathrm{~g} / \mathrm{cc}$ dry density of Puerto Rico clay loam. See Figure 1.

Table 1. Conductivity filter parameters for form-2 dispersive FDTD algorithm to model Puerto Rico clay loam tabulated as a function of moisture and density. A time step size of $\Delta_{t}=20 \mathrm{ps}$ is assumed.

$\begin{array}{ccccccccccc}\mathrm{D}(\mathrm{g} / \mathrm{cc}) & \mathrm{M}(\%) & \log f_{0} & |P| & \epsilon_{r} & b_{0} & b_{1} & b_{2} & a_{1} & a_{2} & X^{2} \\ 1.2 & 5.0 & 8.2 & .75 & 3.490 & .0620899 & -.10508 & .0432 & -1.49882 & .5625 & .0119 \\ 1.2 & 10 . & 8.7 & .75 & 5.007 & .117655 & -.204585 & .0872751 & -1.49703 & .5625 & .0139 \\ 1.2 & 20 . & 8.3 & .80 & 9.063 & .209212 & -.373846 & .165186 & -1.5995 & .64 & .0209 \\ 1.4 & 2.5 & 8.5 & .70 & 3.134 & .0381282 & -.0587637 & .0207773 & -1.39889 & .49 & .0333 \\ 1.4 & 5.0 & 8.5 & .75 & 4.072 & .0744985 & -.122577 & .0483395 & -1.49882 & .5625 & .0183 \\ 1.4 & 10 . & 8.2 & .80 & 5.652 & .12006 & -.213117 & .0932994 & -1.59968 & .64 & .0215 \\ 1.4 & 20 . & 8.5 & .80 & 12.49 & .177603 & -.308787 & .132104 & -1.59874 & .64 & .0160 \\ 1.6 & 2.5 & 8.3 & .70 & 3.718 & .060417 & -.0896431 & .0294616 & -1.39956 & .49 & .0275 \\ 1.6 & 5.0 & 8.5 & .72 & 5.105 & .122843 & -.20338 & .0809494 & -1.43886 & .5184 & .0159 \\ 1.6 & 10 . & 8.5 & .75 & 6.574 & .193683 & -.331925 & .13881 & -1.49882 & .5625 & .0113 \\ 1.6 & 20 . & 8.5 & .78 & 16.06 & .322463 & -.554124 & .233467 & -1.55877 & .6084 & .0091\end{array}$

\section{Conclusions}

A new method has been presented recently for incorporating dispersion into a finitedifference time-domain (FDTD) computational electromagnetics algorithm, which is useful for ground-penetrating radar modeling. A systematic procedure was presented for determining form-1 and form-2 dispersive FDTD model parameters that result in accurate and stable FDTD implementations. The design procedure is difficult because it requires specification and optimization of both the magnitude and phase of a digital IIR over a wide frequency band. In addition, regions of stability for the model coefficients are difficult to obtain. Nevertheless, a design procedure was presented and demonstrated for determining the dispersive FDTD algorithm parameters from measured soil data, resulting in stable and accurate FDTD implementations.

\section{References}

1. R. Luebbers, F. P. Hunsberger, K. S. Kunz, R. B. Standler, and M. Schneider, "A frequency-dependent finite-difference time-domain formulation for dispersive materi- 
als," IEEE Trans. Electromä. Compat., vol. 32, no. 3, pp. 222-227, 1990.

2. R. J. Luebbers and F. Hunsberger, "FDTD for Nth-order dispersive media," IEEE Trans. Antennas Propagat., vol. 40, no. 11, pp. 1297-1301, 1992.

3. T. Kashiwa and I. Fukai, "A treatment by the FD-TD method for the dispersive characteristics associated with electronic polarization," Microwave Opt. Technol. Lett., vol. 3, no. 6, pp. 203-205, 1990.

4. R. M. Joseph, S. C. Hagness, and A. Taflove, "Direct time integration of Maxwell's equations in linear dispersive media with absorption for scattering and propagation of femtosecond electromagnetic pulses," Optics Lett., vol. 16, no. 18, pp. 1412-1414, 1991.

5. O. P. Gandhi, "A frequency-dependent finite-difference time-domain formulation for general dispersive media," IEEE Trans. Microwave Theory Tech., vol. 41, no. 4, pp. 658-665, 1993.

6. D. M. Sullivan, "Frequency-dependent FDTD methods using Z transforms," IEEE Trans. Antennas Propagat., vol. 40, no. 10, pp. 1223-1230, 1992.

7. D. M. Sullivan, "A frequency-dependent FDTD method for biological applications," IEEE Trans. Microwave Theory Tech., vol. 40, no. 3, pp. 532-539, 1992.

8. W. H. Weedon and C. M. Rappaport, "A general method for FDTD modeling of wave propagation in arbitrary frequency-dispersive media," IEEE Trans. Antennas Propagat., 1996. Submitted for publication.

9. W. H. -Weedon and C. M. Rappaport, "A general method for the modeling of arbitrary frequency-dispersive media using FDTD," in Progress in Electromag. Research Symposium, (Seattle, WA), July 1995.

10. C. M. Rappaport and W. H. Weedon, "Efficient modeling of electromagnetic characteristics of soil for FDTD ground-penetrating radar simulation," in IEEE Antennas and Propagat. Soc. Int. Symposium, (Baltimore, MD), July 21-26 1996.

11. J. E. Hipp, "Soil electromagnetic parameters as functions of frequency, soil density, and soil moisture," Proc. IEEE, vol. 62, no. 1, pp. 98-103, 1974. 


\section{Chapter V}

\section{Applications of Infrared Sensors to the task of Landfill Remediation}

Charles A. DiMarzio, Scott C. Lindberg, and Michael J. Rossacci

CENTER FOR ELECTROMAGNETICS RESEARCH

DePartMENT OF EleCTRICAL AND COMPUTER ENGINEERING

NORTHEASTERN UNIVERSITY, BOSTON, MA 02115

Final REPort on Grant DE-FC07-95ID13395

May 14, 1996 


\title{
Applications of Infrared Sensors
}

\author{
to the Task of
}

\section{Landfill Remediation}

\author{
Charles A. DiMarzio \\ Scott C. Lindberg \\ Michael J. Rossacci
}

\section{V-1. Introduction}

Northeastern University, as part of a contract with the Idaho National Engineering Laboratory has undertaken an effort to examine the applications of infrared imagery to the landfill remediation effort. Specifically, the goal was to address the use of infrared imagery in a sensor/data fusion effort to locate, identify, and characterize buried waste. The infrared sensor was expected to provide information on volatile organic compounds (VOC's) in the atmosphere, indicating a broken or leaking underground container.

At the present time, it appears that we will not have appropriate data sets available to be used in the fusion project. We have therefore confined our effort to examination of simple models to determine the level of performance of VOC sensors. Section 2 of this report describes the model and sample results, along with suggestions for the operational use of infrared instruments, complemented with lasers and other light sources.

In addition, we have considered the concept of using the infrared camera along with groundpenetrating radar as a complementary tool for the detection of underground objects. It is well-known that solar heating of the surface of the ground depends on the composition of the soil at and below the surface. This concept has been proposed for the detection of buried mines. With high-power microwave sources, it would be possible to achieve heating comparable to solar heating in a shorter time, and to a greater depth. Section 3 describes our work in this area.

\section{V-2. Infrared Sensing of Volatile Organics}

Many volatile organic compounds have infrared absorption spectra consisting of relatively sharp peaks which are amenable to in situ measurement of portions of the spectra to determine the concentration of one or more-compounds. Considerable success has resulted from work with differential absorption lidar (DIAL), in which the absorption of a laser beam is measured as a function of its wavelength. In the visible spectrum, tunable lasers allow continuous spectra to be obtained over wide bands. In the infrared, lasers such as $\mathrm{CO}_{2}$ operate on many discrete lines, and can be tuned rapidly from one to another. Some of the disadvantages of DIAI are the need for a transmitter, limited spatial coverage because of the need to point the laser and receiver at each desired location, and spectral coverage limited to available laser wavelengths. 
Two new developments suggest an alternative. Infrared cameras of very good sensitivity have been developed to make simultaneous measurements in several thousand (or more) locations at once. Various spectroscopic devices have been developed in such a way as to be compatible with imaging, so that it is now possible to measure a signal as a function of azimuth, elevation, and wavelength. The act of acquisition and processing of such three-dimensional data sets is called "hyperspectral imaging." It has found applications in satellite remote sensing, in medical imaging, and military activities.

The development of this field suggests a passive concept analagous to DIAL. Using the natural ("Black-body") radiance of an object or scattered solar radiation, it seems reasonable to ask whether it would be possible to determine changes in absorption between that object and a hyperspectral imager. The fundamental difference between this technique and DIAL is that the filtering is done at the receiver rather than the source.

For example, in DIAL, laser light is spectrally concentrated at the wavelength of interest, yielding a significant scattered radiance, $L$, from the object. For a laser power, $\Phi$ incident on an area $A$ of a target of diffuse reflectivity $Q_{2}$, the scattered radiance will be

$$
L=\rho \frac{\Phi}{A}
$$

Powers of Watts in areas of a few square centimeters are common, and reflectivities are often a few percent per steradian. Thus, the radiance may be of the order of 1000 Watts/ meter $^{2} /$ steradian.

of $^{-}$

On the other hand, filtering at the reciever, using scattered sunlight provides a measured radiance

$$
L=\rho E_{\lambda}[\text { solar }] d \lambda,
$$

where the solar irradiance, $E$ [solar], is never much more than 1000 Watts per square meter spread over the entire spectrum, and $E_{\lambda}$ [solar] has a maximum value not often greater than 20 Watts per square meter per micrometer at 3 to 5 micrometers (Mid-Infrared or MIR) and 1 Watt per square meter per micrometer at 10 to 14 micrometers (Far-Infrared or FIR). We must set the filter bandwidth $d \lambda$ to be at least comparable to the expected linewidth. Assuming a line of 100 nanometers width, which is perhaps unrealistically large,

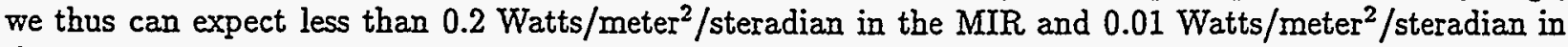
the FIR. We thus begin with a disadvantage of at least 4 orders of magnitude compared to DIAL, but with considerably simpler hardware.

\section{V-2.1. Simple Models}

To explore the possibilites of using IR detection of VOC's, we have enhanced a program originally developed for scanning lidar, which calculated the radiance of ground and sky, including scattered sunlight, to be used as noise contributions. A program element for computing the intersection of the line of sight with targets was modified to model a plume.

For each pixel in a scene, the program computes the intersection with the ground and the plume if applicable, and computes,

- the solar irradiance incident on the ground, attenuated by the plume if the pixel is in the shadow of the plume, and

- the radiance of the ground, as the sum of the self-radiance and the scattered solar irradiance,

- the radiance of the sky behind, in, and in front of, the plume. 
Each of these is attenuated by the atmosphere and the plume as appropriate.

The pictures in Figure 1 illustrate the function of the program. Realistic camera parameters were used for each wavelength. A nearly opaque plume was carefully chosen to show all the features of the program. Note that the shadow of the plume is obvious in the visible and MIR pictures, because a significant portion of the ground radiance arises from scattered sunlight. On the other hand, in the FIR picture, very little of the ground radiance results from scattered sunlight, so the shadow can not be seen.

The ground is hotter than the sky, and appears brighter in the IR images. It appears darker in the visible image because both sky and ground radiance are dominated by scattered sunlight and the sky's scattering is stronger. The plume was made cooler than the ambient air, so it shows up darker than either the sky or the ground in the IR pictures. Sunlight scattered from the plume was not included, so it appears dark.

Although the model uses a very simple geometry and would not be useful for modelling a dynamic situation with multiple plumes of different constituents in a varying atmosphere and terrain, it is sufficent to determine the capability of hyperspectral IR cameras to detect and perhaps quantify VOC's.

\section{V-2.2. Performance Predictions}

Two applications for this technology present themselves, and we will develop models of each. In the first, remediation activity at a particular site is planned or under way, and multiple sensors are in place to assist in identifying the buried material. These may include one or more ground-penetrating radars, electromagnetic sensors such as EM-61 and EM-31, and magnetic-field sensors. The functions of the VOC sensor are to

- assist in identifying buried material by locating plumes from leaking containers, and

- assist in protecting the workers by providing a timely alert if VOC levels increase during the work.

In the second scenario, workers enter an area where VOC's are known to exist. The goal of the sensor is to monitor the site continuously so that workers may be removed from the area or don protective clothing if the level of VOC rises toward a threshold.

The major differences are the following:

- In the first case the sensor is an integral part of the measurement program while in the second it is expected to operate relatively autonomously and unobtrusively until it detects the threshold signal.

- In the first case the sensor can be moved to cover specific areas as part of the measurement process, while in the second, it must survey the entire site continuously and autonomously.

- In the first case the plume is likely to be small in spatial extent, so that it can be identified against a background, while in the second case, it is more likely to be distributed over the entire area, so that the variations in concentration from pixel to pixel will be small.

- In the first case, it is important to identify the material and locate the source, with concentration being less important, while in the second, the material may be.known in advance, and location is relatively unimportant, but concentration is critical. 


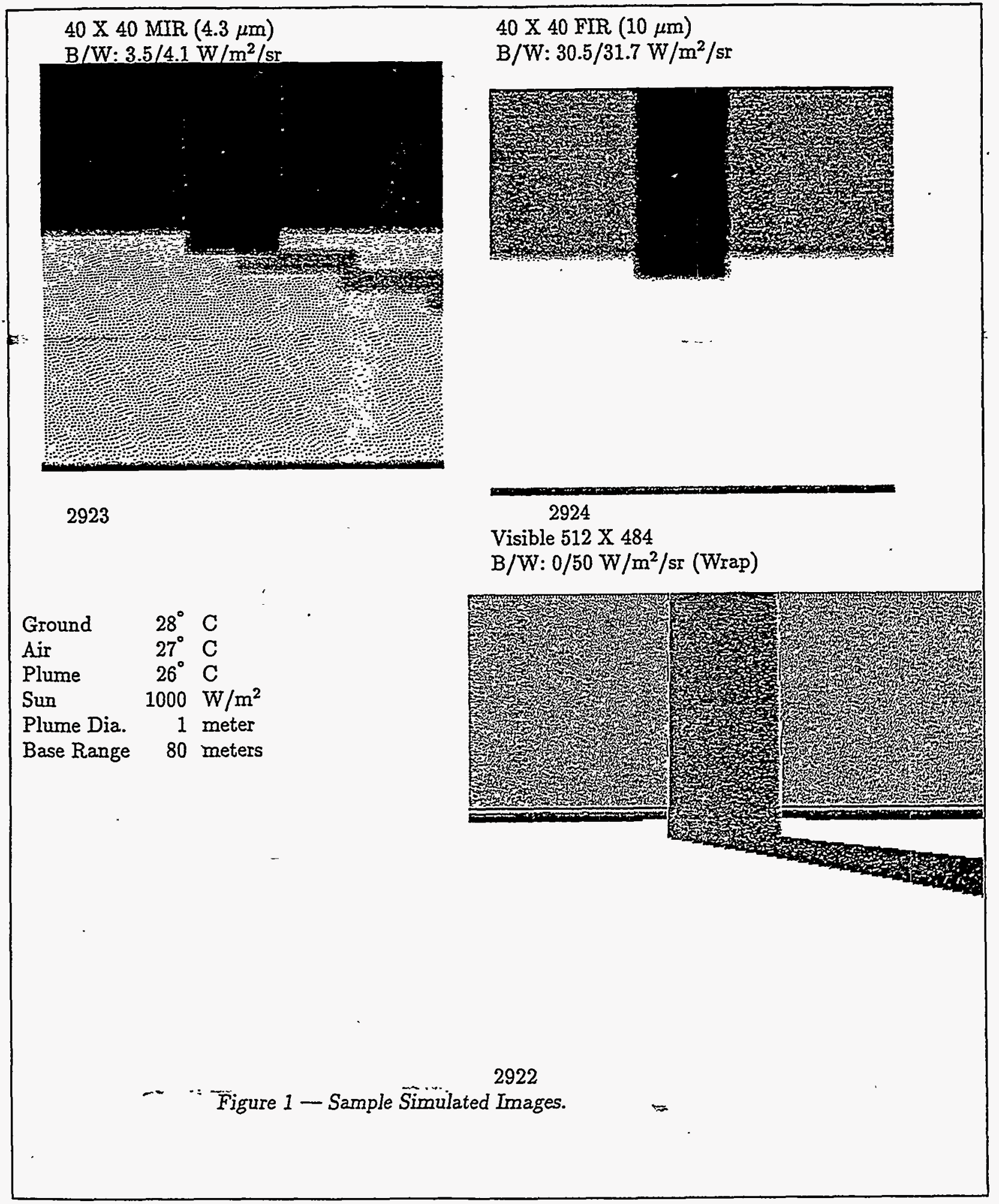

We consider two examples, one for each of these scenarios, and show calculations for the first. In this case, we have a 5 meter diameter plume from a particular site. The field of view consists of 40 pixels in each direction for a total exent of 0.558 radians in each direction. The plume is located 80 meters away, 
in the center of the field of view, and the sun is to the left and in front of the camera. The wavelength is near 4.3 micrometers. No absorption data have been available, but we will estimate $5 \mathrm{~dB} / \mathrm{km}$ as a typical absorption coefficient. The ambient temperature of the atmosphere, including the plume is 27 degrees $\mathrm{C}$, and the ground is one degree warmer. The ambient atmospheric attenuation is $3 \mathrm{~dB} / \mathrm{km}$, and the solar constant is 1000 Watts per square meter.

The results are shown in Figure 2. In the bottom plot, a horizontal line through the plume (line 18 ) is shown. In the top plot, a line below (in the image) the base of the plume (line 19) is shown. The distinguishing feature here is the shadow cast by the plume on the ground. The minimum sensitivity is assumed to be $2.5 \times 10^{-4}$ Watts per square meter per steradian [Sonnenfroh, et. al.]. In both lines, the plume is clearly visible.

In line 15-(not shown), which has a sky background, the plume is not visible, because it has the same temperature as the atmosphere. Any light which it absorbs is replaced by light which it radiates. Detection of a plume against a sky background will be impossible if no temperature contrast exists.

The same case is shown in Figure 3 for a center wavelength around 10.5 micrometers. It will be noted that the solar spectrum is low at this wavelength, and does not produce a shadow as it did in the MIR case. Other than that, the details are similar, although the actual radiances are higher.

In the second case, the plume is 50 meters wide, which is a substantial fraction of the image. Attenuation will be about 10 times greater, and either detection will be relatively easy, or it will be possible to detect lower concentrations.

The results may be understood by considering the following: Sunlight is assumed to have a black body spectrum associated with a temperature of $5000 \mathrm{~K}$. Scattered sunlight has a spectrum proportional to this one, but reduced in magnitude by an appropriate amount to indicate the scattering from ground, clouds, or other objects. The blue sky is simulated by the solar $(5000 \mathrm{~K})$ spectrum, reduced in magnitude, and multiplied by a Rayleigh-scattering factor proportional to the inverse fourth power of wavelength. All of these are summarized in Figure 4. The figure shows the scattered sunlight from "blue sky" and "clouds or terrain," as well as a 300-K black-body spectrum identified as "night sky." The latter is, of course observed for any object at a temperature of 300 Kelvins, including the ground.

For the "thick" objects (sky, plume, etc.), the indicated values are for infinite thickness. For finite regions, the computation requires solution of the radiative transport equation. The result is a contribution

$$
L_{r_{1}, r_{2}}=L_{0, \infty} \exp \left[-\alpha_{1} r 1\right]\left\{\exp \left[-\alpha_{2}\left(r_{1}\right)\right]-\exp \left[-\alpha_{2}\left(r_{2}\right)\right]\right\}
$$

If the plume is at the same temperature as the ambient air, then an IR sensor viewing the sky will observe the same black-body signal with or without the plume. Specifically, any light from the sky behind the plume which is attenuated by the plume will be replaced by black-body radiation from the plume itself.

Thus, the plume will only be visible if there is an additional light source behind the plume. This can occur if, for example, the ground temperature is higher than the ambient air temperature, or if sunlight scattered from the sky or ground background is also detected.

Scattered sunlight from a cloud or terrain is shown in Figure 1 to be well below the black-body radiation in the FIR band, but comparable to it in the MIR. Thus, if a plume of gas has an absorption signature in each band, it may be preferable to use the MIR band to take advantage of scattered sunlight. 

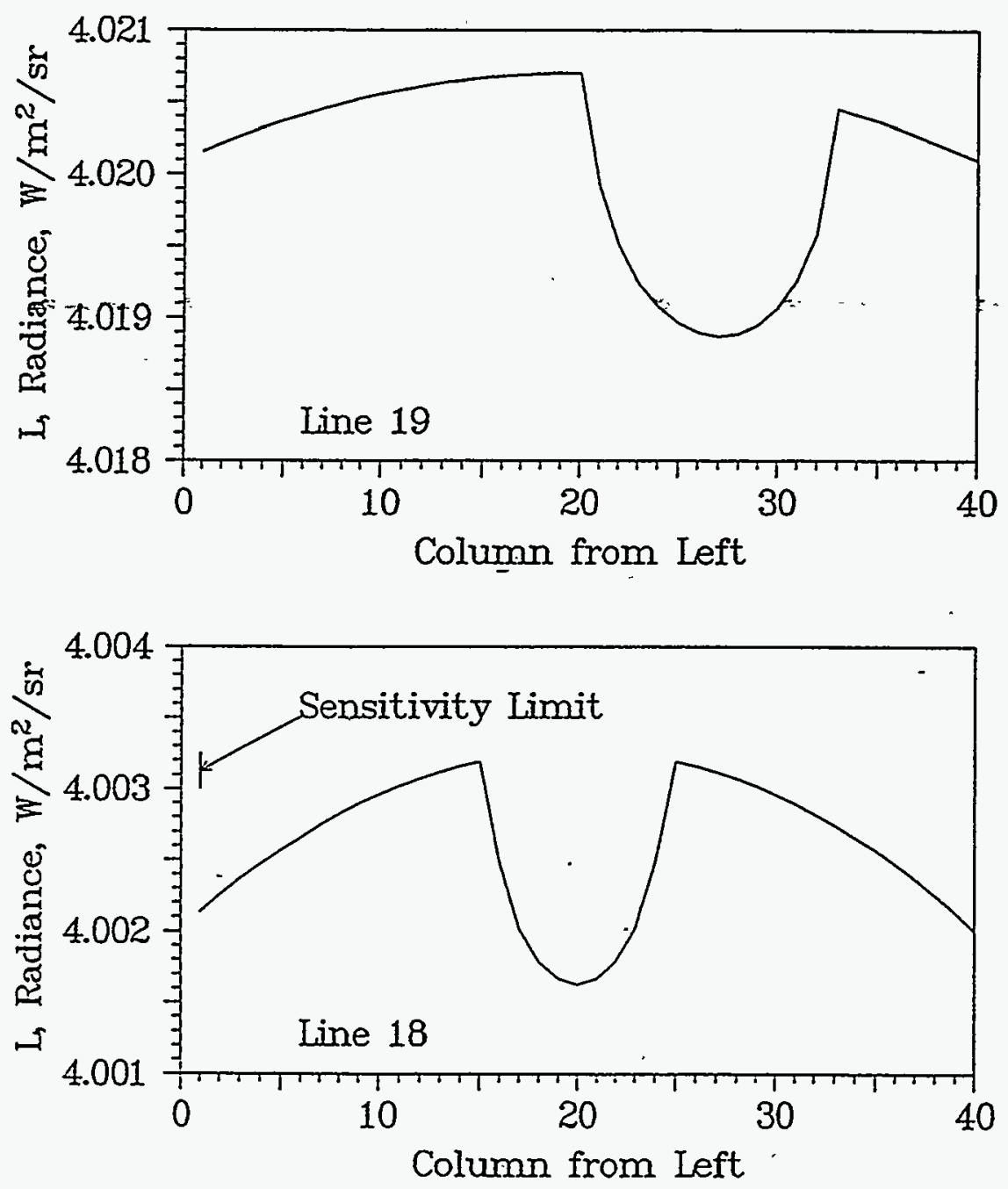

Figure 2 - Slices Through Images at 4.3 micrometers.

\section{V-3. Infrared Sensing of Buried Objects}

During the performance of this work, another opportunity suggested itself. A multiple-modality sensor, in which a high-power microwave transmitter is combined with an infrared camera might be useful for deteting underground objects relatively near the surface. The microwave transmitter would introduce energy 

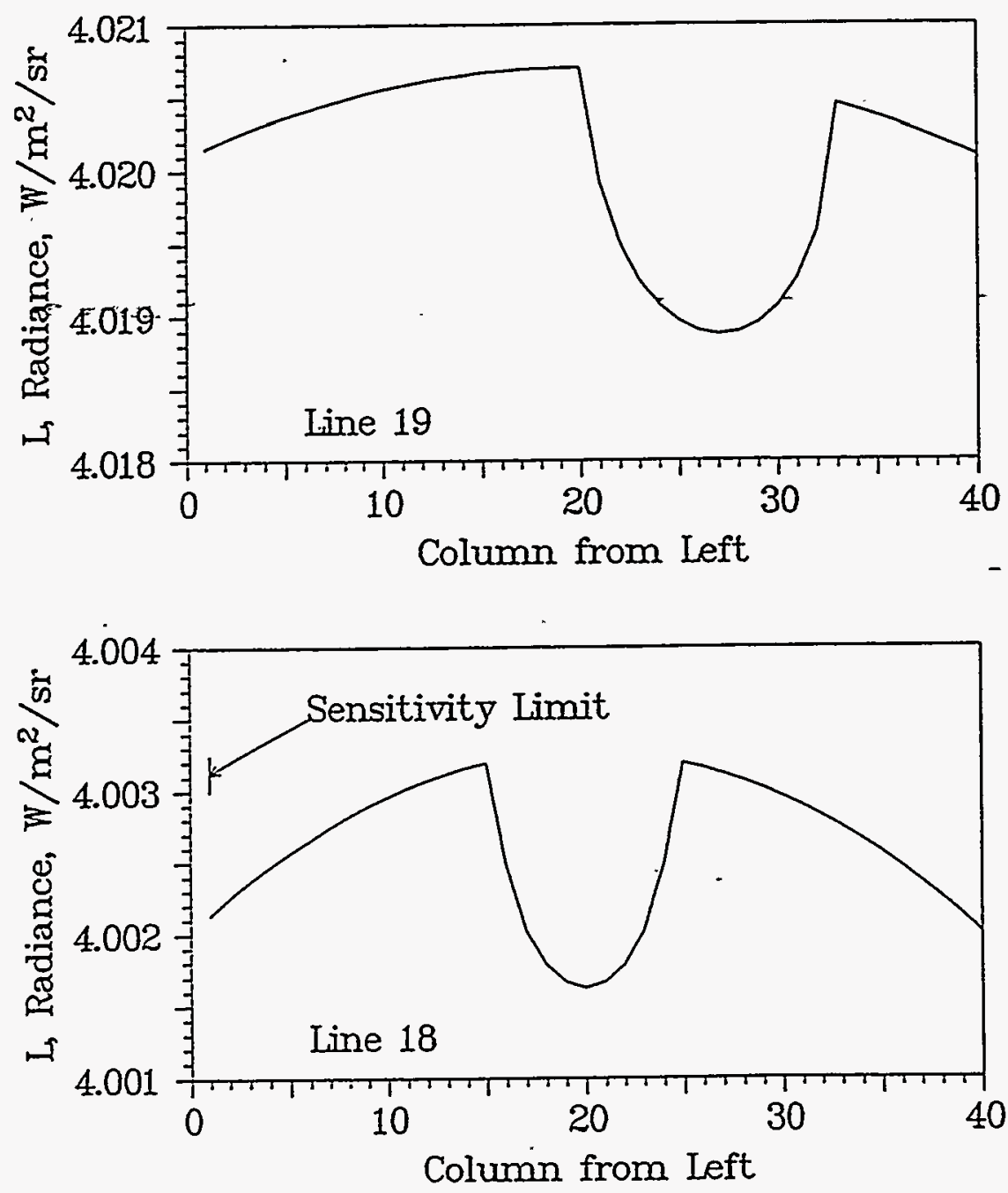

Figure 3 - Slices Through Images at 4.3 micrometers.

into the ground which would be absorbed at different rates by buried objects than by the surrounding soil. The resulting differential heating would be sensed by the infrared camera, and an image of the underground object produced.

While careful modelling will be required to evaluate performance, and optimize parameters, the basic soundness of the approach is suggested by two analyses: 


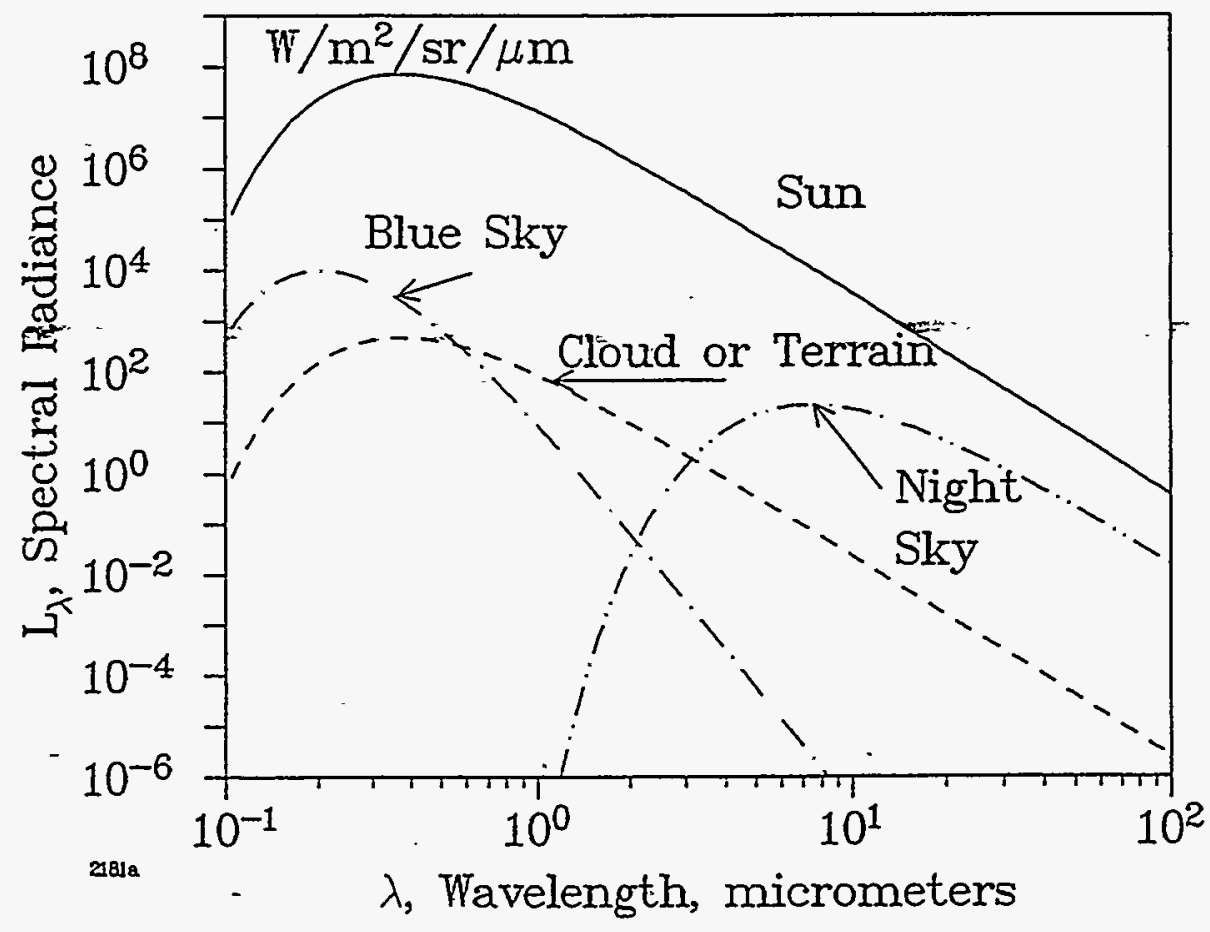

Figure 4-Spectra of Natural Scenes.

First, it has been shown [ $[\mathrm{i}$, et. al.] that differential heating by sunlight can be measured in a time scale of minutes. The nominal 1000 Watts per square meter of sunlight are concentrated in the optical region of the spectrum and are absorbed at the surface of the ground. The image is weak because it is produced entirely by heat transfer, and probably noisy, because of uneven absorption of sunlight at the surface. A microwave wavelength can be selected for penetration to the desired depth where mines are expected, thus making use of the dielectric contrast of the mine as well the contrast in its thermal parameters.

Secondly, we can perform a simple intuitive analysis. If an object absorbs as little as 1 Watt per square centimeter and is located 1 centimeter below the surface, assuming as a worst case the specific heat of water, it will heat by about 0.25 Kelvin per second. The resulting radiance change caused by a temperature change of $0.25 \mathrm{Kelvin}$ at $300 \mathrm{Kelvin}$ is about $0.02 \mathrm{~W} / \mathrm{m}^{2} / \mathrm{sr}$ in the 3-to-5-micrometer (MIR) band and $0.25 \mathrm{~W} / \mathrm{m}^{2} / \mathrm{sr}$ in the 8-to-12-micrometer (FIR) band. A good infrared camera [Sonnenfroh, et. al.] has a sensitivity of $2.5 E-4 \mathrm{~W} / \mathrm{m}^{2} / \mathrm{sr}$, offering two to three orders of magnitude margin. Intuitively, the preference 
would be for the FIR band, because the signal is stronger and because scattered sunlight will not contaminate the measurement.

We have developed a program [Rossacci] which calculates the amount of energy absorbed from an electromagnetic field by a heterogeneous material and then solves the heat equation to determine the temperature as a function of time and position. This program was originally developed for medical applications, and is presently being considered as a model for mine detection using a microwave source and infrared camera.

\section{V-R. References}

Li, P., A. Maad, F. Moshrary, M. F. Arend, and S. Ahmed, "Infrared Imaging of Buried Objects by Thermal Step-Function Excitations" Applied Optics 34, 25, 1 September 1995. Pp. 5809-5816.

Rossacci, Michael J., "Temporal and Spatial Variations of Temperature in the Laser Heating of a Heterogeneous Turbid Medium," Presented at the IEEE Student Paper Competition, Boston University, Boston, MA 26 April 1996.

Sonnenfroh, David M., B. David Green, William J. Marinelli, and W. Terry Rawlins, "A Novel Infrared Imaging Spectroradiometer for Passive Remote Detection of Clouds," presented at Cloud Impacts on DOD Operations and Systems 1995 Conference, U. S. Air Force Phillips Laboratory, Hanscom Air Force Base, MA. 24-26 October 1995. 


\section{Chapter VI}

\section{Lidar for Detection and Monitoring of Fugitive Dust Emissions}

Charles A. Dimarzio and Scott C. Lindberg

CENTER FOR ELECTROMAGNETICS RESEARCH

DEPARTMENT OF ELECTRICAL AND COMPUTER ENGINEERING

NORTheastern University, Boston, MA 02115

Final Report on Grant DE-FC07-95ID13395

May 14, 1996 


\title{
Lidar for Detection and
}

\section{Monitoring of Fugitive}

Dust Emissions

Charles A. DiMarzio Scott C. Lindberg

\author{
VI-1. Introduction
}

Northeastern University is presently working on several tasks related to landfill remediation. One part of the effort is to use our knowledge of lidar for dust monitoring, gained through previous work on other programs, to make recommendations for lidar concepts which could be applied during landfill remediation. This is an interim report on the status of that work.

Section 2 of this report is a brief tutorial on the concept of lidar dust monitoring including some history of other efforts. Section 3 describes some of the scenarios in which dust monitoring could be important in landfill remediation. Section 4 proposes lidar specifications which could meet the needs described in section 3. Section 5 describes an existing lidar similar to one which might meet these needs. Section 6 consists of conclusions and recommendations.

\section{VI-2. Overview of Lidar Dust Monitors}

Lidars may be divided into three classes according to the detection technique as shown in Figure 1.

\section{Direct Detection}

The first of these, called autodyne, is the simplest. Light from a laser is incident on a target, and the reflected or backscattered signal is detected. This concept does not retain any phase information, and has not found much application to Doppler measurements of frequency. It is useful for aerosol measurements, but has a lower signal-to-noise ratio than coherent detection. The name autodyne is used by comparison to the other two techniques, and probably was introduced by Shapiro [Fluckiger, Kreys, and Shapiro]. The technique is also called "direct detection," or "incoherent detection."

Backscatter LIDAR (Light Detection and Ranging) can be used to detect and track aerosol pollutants in the atmosphere. A LIDAR consists of a transmitter laser, some optical components, and a detector. The transmitter is normally pulsed. The transmitted light is directed through a telescope, and usually a scanner toward the target. 


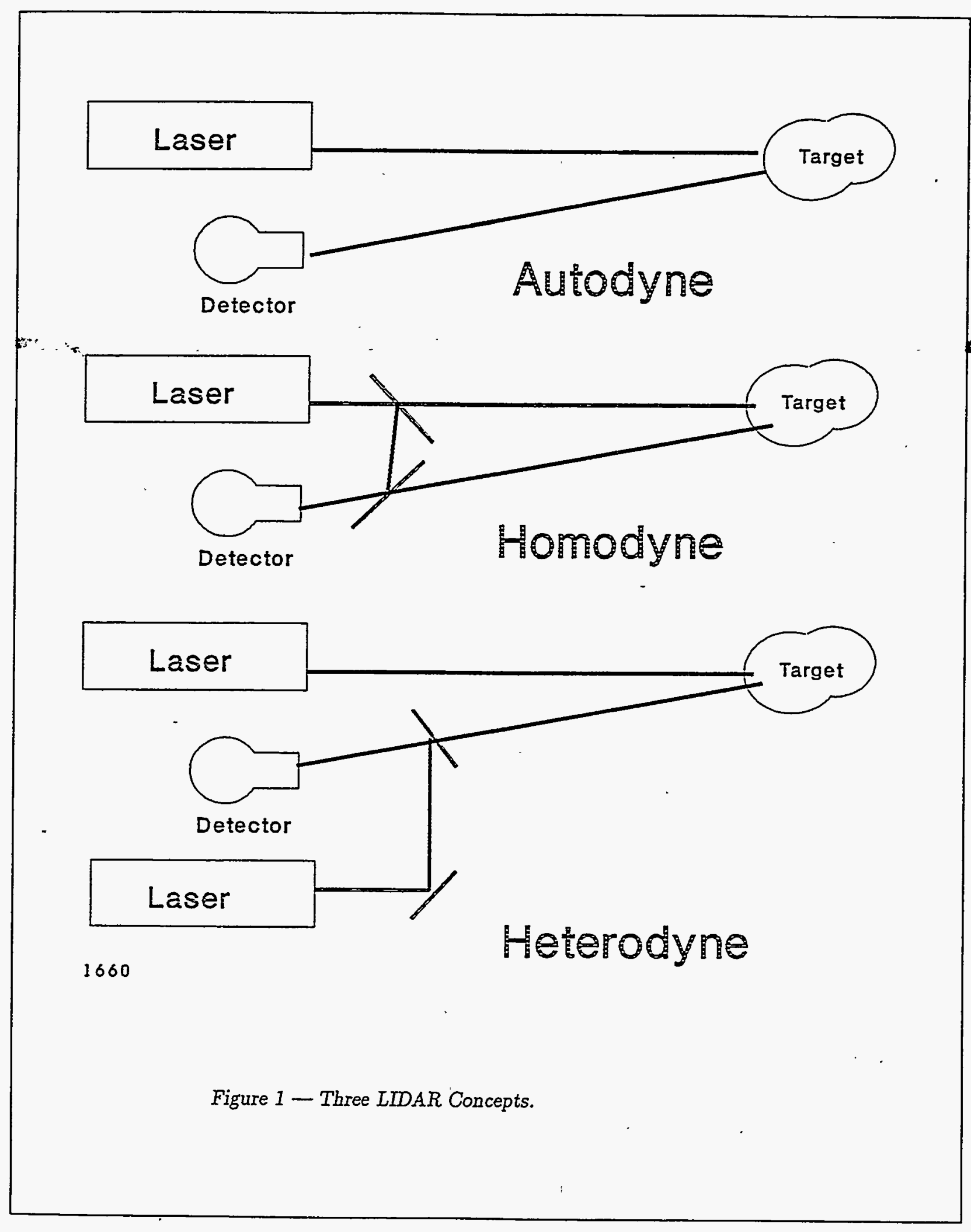

Light is scattered back from the target toward the LIDAR. Because of the short wavelength, aerosols 
with diameters from a few microns scatter enough light to provide a sufficient target. The backscattered light level from each particle is proportional to the cross-sectional area, the incident light level, and the "scattering efficiency" of a particle with its particular size, shape, and composition.

Light from the target is collected by the telescope ind directed toward the detector. The detector is normally time gated to measure the signal from different ranges.

Performance of LIDAR for this application is determined by the wavelength of the laser, the scattering characteristics of the material, the absorption of light by the intervening atmosphere, the transmitter pulse amplitude and duration, and the aperture of the receiver.

\section{Coherent Detection}

In the second, or homodynte system, thè returned signal is mixed with a sample of the transmitter laser power, resulting in a signal from the detector at the difference frequency between the unshifted laser and the Doppler shifted return signal. This approach is particularly attractive for continuous-wave (CW) transmitters, but is also useful for pulsed systems in the master-oscillator-power amplifier (MOPA) configuration [DiMarzio, Harris, Bilbro, Weaver, Burnham, and Hallock]. In this case, a modulator chops the $\mathrm{CW}$ laser to produce pulses, which are then amplified in the transmitter path. The laser amplifier may be placed in the common path of the transmitter and receiver, in which case it may function as a preamplifier for the returning signal in addition to providing the transmitted pulse energy [Simon]. For this to occur, it is important that the gain in the laser medium not be completely expended in amplifying the transmitted pulse. Normally, some residual gain remains for a period of time after the pulse but before the next pumping. cycle.

The heterodyne configuration is similar to the homodyne one, except that the reference beam on the detector is derived from a separate laser, normally offset in frequency from the transmitter. As explained later, this allows sign discrimination of the Doppler signal. A variation frequently used involves generating the offset reference beam from the transmitter laser with a Bragg Cell. This is occasionally referred to as "offset homodyne" [Nordstrom and Berg].

All of these techniques are referred to collectively as "coherent detection." In addition to providing a reference for the measurement of the Doppler frequency, they also produce a large reference beam on the detector, resulting in quantum-limited detection. This is particularly important at long infrared wavelengths where direct detection is almost never quantum-limited. Because of their power, efficiency, relative eye safety, and coherence, carbon dioxide lasers at wavelengths near 10 micrometers have been the transmitters of choice since the 1970's, although recently solid-state lasers near 2 micrometers have come to be of interest [Kane, Byer, and Zhou].

In the present work, we will dwell mainly on the homodyne detection technique. Velocity measurements with a laser Doppler anemometer may be understood with the aid of Figure 2, which shows a typical idealized laser radar. Most of the energy from the laser transmitter is directed to the object being used as a target, which may be either a hard target, artificially generated aerosols, or the dust particles normally suspended in the atmosphere. In general, the scatter from molecules will be too weak to be measured at - infrared wavelengths. This turns out to be fortuitous for Doppler measurements, for the Doppler broadening caused by Brownian motion of the molecules is hundreds of time larger than typical ambient wind velocities.

A smaller fraction of the transmitter energy is diverted toward the infrared detector and is used as a reference beam. Some of the energy backscattered from the target, which is delayed in time and Doppler shifted according to the line of sight component of the target's velocity, is also incident on the detector. 


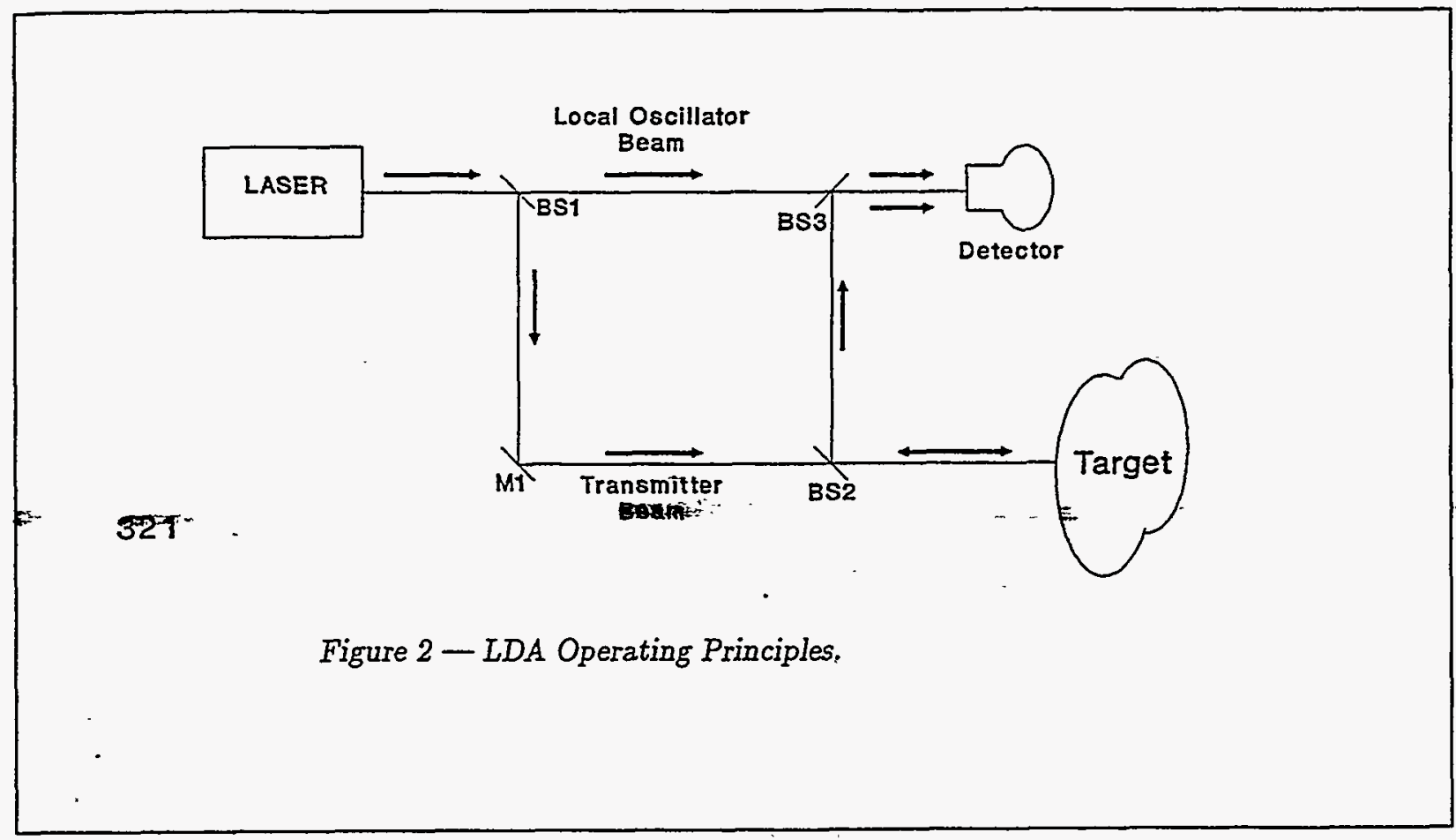

Figure 3 illustrates the component of velocity which is actually measured by the lidar. Single velocity measurements yield only that component which is along the line of sight of the laser beam.

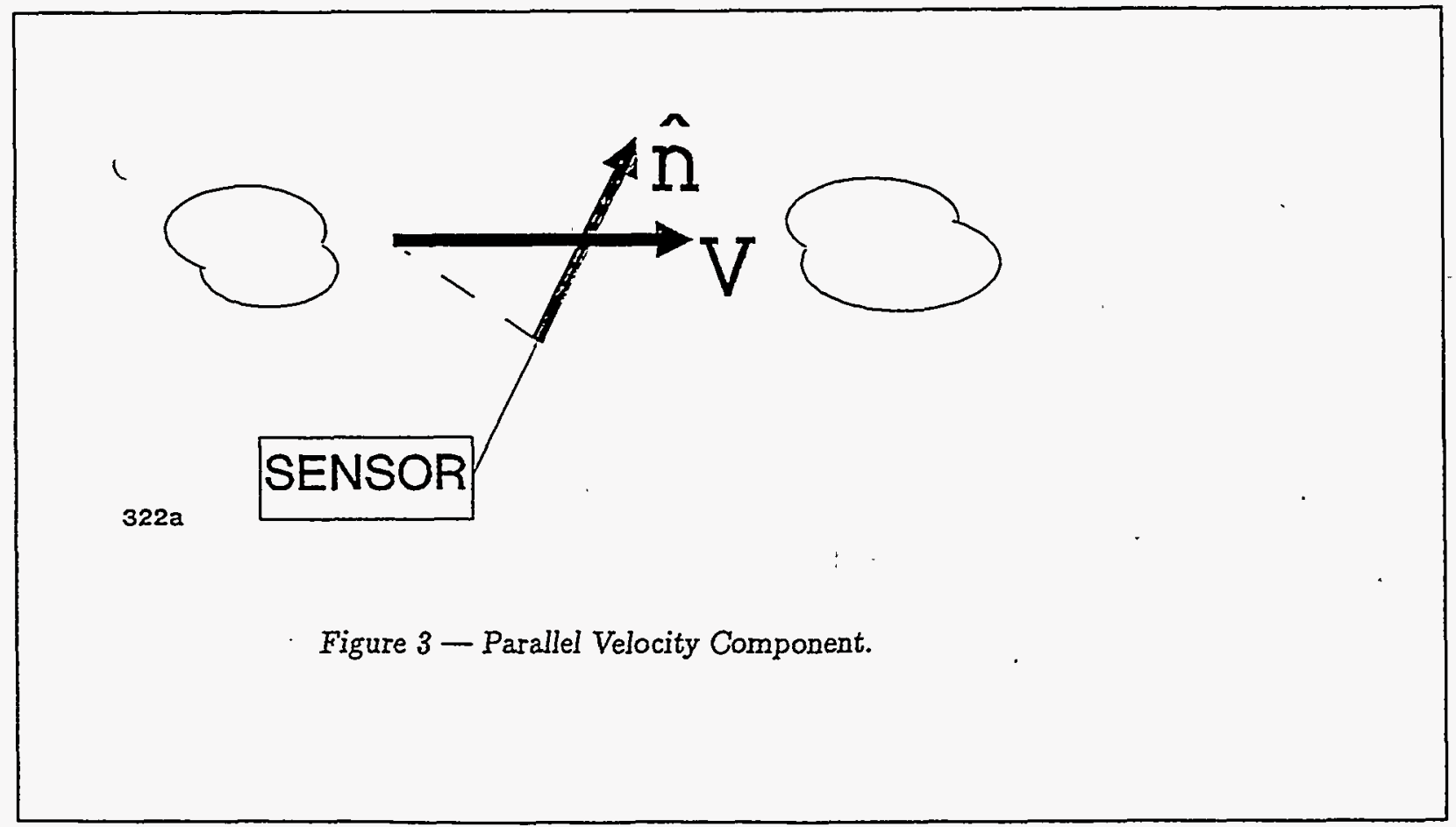


As described above, there are two beams incident on the detector. The first is a reference beam of approximately one milliwatt in power at the transmitter frequency. The second is the signal return beam which is offset from the transmitter frequency by the Doppler frequency of the target. This beam is typically at a level of ten femtowatts. These two beams, as shown in Figure 4, are detected by the square law detector, resulting in a peak at the difference frequency. This corresponds to the Doppler shift generated by the target motion. A typical return is shown in Figure 5 . This return was obtained with a $C W$ diode laser at a wavelength of 810 nanometers. The use of a coherent lidar adds a layer of complexity to measuring dust concentration, but the increased sensitivity may be an attractive return for the added effort.

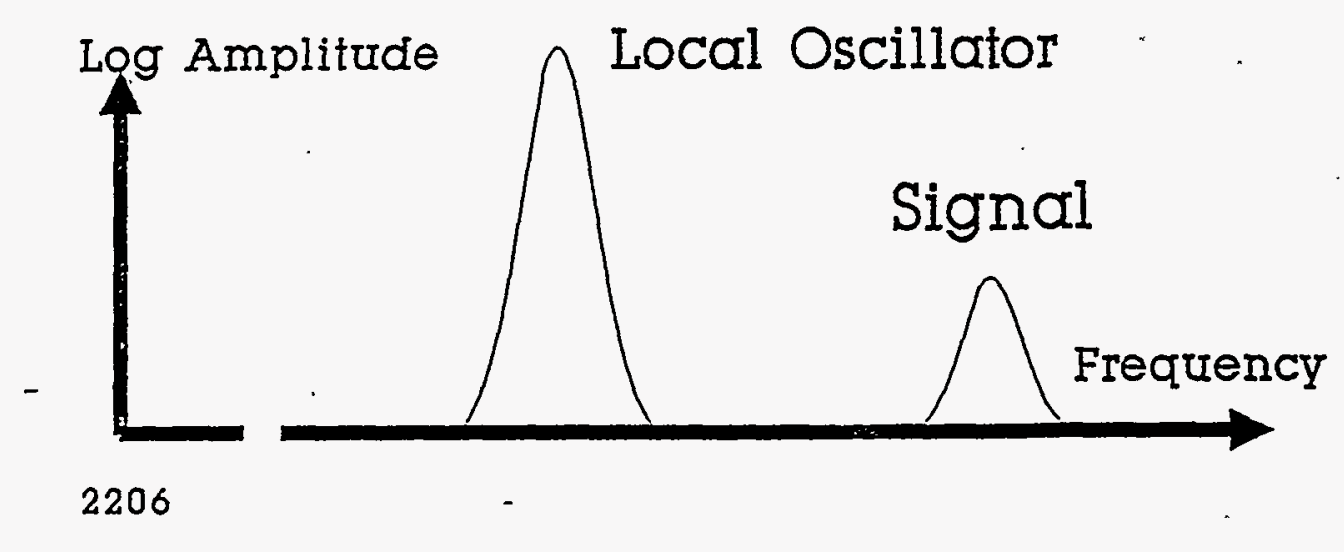

Figure 4-Beams Incident on Detector.

\section{VI-3. Requirements for Remote Dust Monitors}

We will consider two different situations. In the first, work is being done at a small, well-defined site, and the goal is to determine if sub-visual dust is being generated at the site, with the possibility of being propagated away from the site. In the second case, a less concentrated level of work is being done over a larger area, and the goal is to determine whether small amounts of dust are being generated, and if so, to locate the sources. The former will be addressed by what will hereafter be called the short-range lidar, and the latter by the long-range lidar.

VI-3.1. Common Requirements

Several requirements are common to both the short-range and long-range systems. 


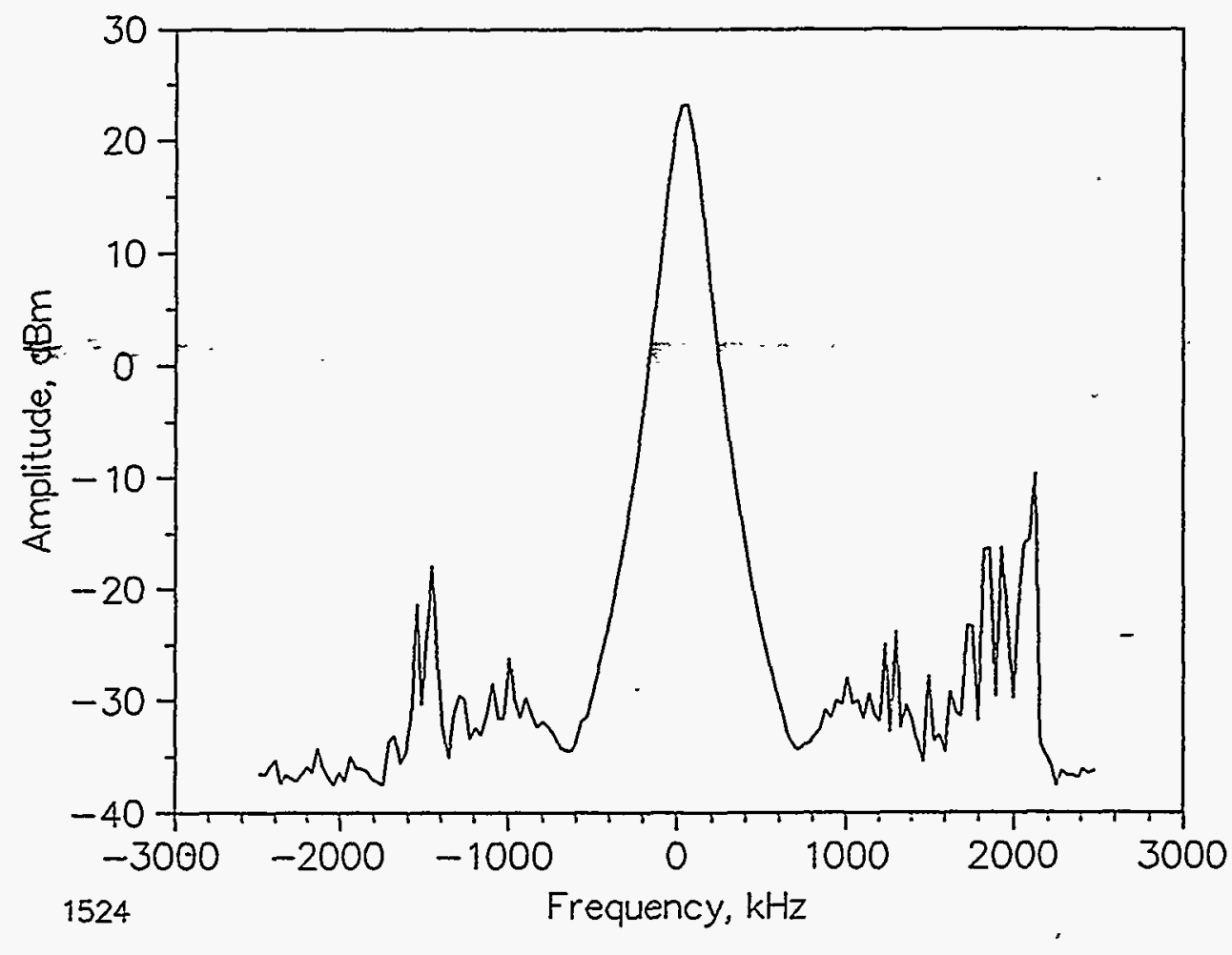

Figure 5-Typical LDA Beat Signal.

\section{VI-3.1.1. Sensitivity to Backscatter}

Large, dense dust clouds are readily visible to the unaided eye, and lidar is unlikely to be competitive with an alert observer in identifying and tracking such emissions. The goal then, is to design a lidar system for detection of those sub-visual clouds, which are not easily detected by eye. The infrared wavelengths of most Doppler lidars are particularly suitable for detecting large (few micrometer) aerosols against a background of molecular scatter. We begin with the requirement that the lidar must be able to detect the ambient aerosol typically found in continental environments. At a wavelength of 10 micrometers, this is about $10^{-8} / \mathrm{m} / \mathrm{sr}$, and at the short end of the infrared spectrum, it is somewhat larger. 


\section{VI-3.1.2. Velocity Coverage}

Significant advantages in sensitivity may be possible with the use of a Doppler-Lidar, because of the advantages of coherent detection; increased signal strength and narrow bandwidth. It is thus important to specify the Doppler coverage.

At the upper end of the Doppler spectrum is the highest wind speed which will be encountered along the line of sight. Some Doppler lidar components are capable of detecting Doppler shifts of satellite velocities, and almost all are capable of measuring aircraft velocities, so the upper limit is not important for the present purpose.

At the low end, we must detect particles moving slowly across the line of sight. If a particle were perfectly stationary, the return would be lost in the low-frequeney-noise of the local oscillator. Of course, for a particle to come into the field of view of the lidar requires some motion. We propose a lower Doppler limit of $1 \mathrm{~m} / \mathrm{s}$.

\section{VI-3.1.3. Operational Issues}

The lidar must be designed to be moved from one site to another, although for the intended purposes, true portability is not a major issue. Setup time must be kept to a minimum if the lidar is to be useful for routine work. We propose the goal that the system be movable by pickup truck, and that a variety of lifting points be provided.

We assume that, if necessary, liquid nitrogen can be provided to cool the detector and canisters of laser gas can be changed on site as needed.

\section{VI-3.2. Short-Range Lidar}

The purpose of the short-range lidar is to detect dust in the immediate vicinity of a work site. Generally we want assurance that there is no dust above a certain level in the work area. Thus, we require relatively dense coverage, although the ability to locate the dust precisely is not particularly important. Range and azimuth must be.treated differently in this respect. For either a pulsed or CW lidar, the signal at a specific range is the integral of contributions from ranges within the resolution cell. Thus, as the range resolution becomes large, we lose the ability to locate a small dust cloud in range, but the strength of the signal is only reduced by the ratio of the cloud depth to the range resolution. We selected 10 meters for the range resolution for the short-range system. For angular spacing, the issue is that the narrow diameter laser beam (perhaps 200 microradians) could miss clouds between adjacent "fingers" of the scan pattern. Thus we specify the angular spacing to be 1 meter at maximum range. Given the confined nature of.a work site, we suggest a maximum range of 150 meters.

\section{VI-3.3. Long-Range Lidar}

For the long-range lidar, the major objective would be to locate and track small dust clouds to determine their source and extent. For this purpose, we envision a 1-km maximum range with 15 meters of range resolution. The goal would be to have a comparable resolution in the transverse direction at maximum range. If we establish the update rate to be 15 seconds, then a cloud will move one range resolution element 
per scan at a mean velocity of 1 meter per second, and a cloud travelling at 10 meters per second across a 2- $\mathrm{km}$ site with the lidar in the center would be observed about 12 times during some three minutes.

\section{VI-3.4. Summary}

The requirements are summarized in Table 1.

Table 1

Lidar Requirements

\begin{tabular}{|l|l|l|}
\hline Requirement & Short-Range & Long-Range \\
\hline Sensitivity & Ambient Aerosol & Ambient Aerosol \\
\hline Minimum Doppler & Below 1 m/s & Below 1 m/s \\
\hline Transportability & Light Truck & Light Truck \\
\hline Logistics & $\begin{array}{l}\text { Liquid Nitrogen, Laser Gas Can- } \\
\text { isters OK }\end{array}$ & $\begin{array}{l}\text { Liquid Nitrogen, Laser Gas Can- } \\
\text { isters OK }\end{array}$ \\
\hline LN2 Refill Time & $\geq 8$ hours & $\geq 8$ hours \\
Time per Gas Can & $\geq 24$ hours & $\geq 24$ hours \\
Startup Time & 15 Minutes, one person & 15 Minutes, one person \\
& & \\
& & \\
Power & $110 \mathrm{~V}, 1 \phi<15 \mathrm{~A}$ & $110 \mathrm{~V}, 1 \phi<15 \mathrm{~A}$ \\
\hline Range & $150 \mathrm{~m}$ & $1000 \mathrm{~m}$ \\
\hline Range Resolution & $10 \mathrm{~m}$ & $15 \mathrm{~m}$ \\
\hline Angle Coverage & $90 \mathrm{degrees}$ & 360 degrees \\
\hline Angular Density & $1 \mathrm{mmeter}$ transverse at maximum & $15 \mathrm{~m}$ Ifters transverse at maximum \\
\hline Cycle Time & $30 \mathrm{sec}$ & $15 \mathrm{sec}$ \\
\hline
\end{tabular}




\section{VI-4. Lidar Specifications for Monitoring Dust}

Many different lidars can be proposed to meet the specifications in Section 3. One issue is the choice of wavelength. For operations such as this, eye safety and the goal of detecting large particles suggest the use of infrared. The two likely candidates are based on the carbon dioxide laser at a wavelength of around 10 micrometers and the Holmium YAG at around 2.1 micrometers. These two lasers are well-developed, high power, and operate in regions of good transmission through the atmosphere.

Another critical decision is the detection concept. We slightly favor Doppler lidar, because of the enhanced sensitivity of coherent detection, and the need to detect very low concentrations of aerosol. We are, however, concerned about the added complexity of Doppler lidar over direct detection, and will address this issue in further study.

Third in the decision process is the issue of ranging. We-select a pulsed lidar for the long range system; because it has the required range resolution but does not work in the first few range gates. For the short-range lidar, we select a CW lidar in which ranging is achieved by changing the telescope focus. This concept has no minimum range, but does not work well near the far field of the telescope $\left(\pi D^{2} / 4 \lambda\right)$.

Table 2 -

Short-Range Lidar Specifications

\begin{tabular}{|l|l|l|}
\hline Requirement & $\mathrm{CO}_{2}$ & Ho:YAG \\
\hline Concept & $\mathrm{CW}$ Doppler & CW Doppler \\
\hline Power & 5 Watts & 5 Watts \\
\hline Aperture & $20 \mathrm{~cm}$ & $20 \mathrm{~cm}$ \\
\hline Azimuth Scan & $90 \mathrm{deg} / 30 \mathrm{sec}$ & $90 \mathrm{deg} / 30 \mathrm{sec}$ \\
\hline Elevation & Manual Set & Manual Set \\
\hline Range Scan & $0-200 \mathrm{~m}$ in $0.25 \mathrm{sec}$ & $0-200 \mathrm{~m}$ in $0.25 \mathrm{sec}$ \\
\hline Passband & $200 \mathrm{kHz}$ to $10 \mathrm{MHz}$ & 1 to $50 \mathrm{MHz}$ \\
\hline
\end{tabular}

The lidar specifications are shown in Tables 2 and $3 .=$

\section{VI-5. Existing Lidar for Dust Monitoring}

It may be useful to use an existing lidar design as a starting point. Furthermore, the lidar described below may be available for use as a test bed for preliminary field tests. 


\begin{tabular}{|c|c|c|}
\hline \multicolumn{3}{|c|}{$\begin{array}{c}\text { Table } 3 \\
\text { Long-Range Lidar Specifications }\end{array}$} \\
\hline Requirement & $\mathrm{CO}_{2}$ & Ho:YAG \\
\hline Concept & Pulsed Doppler & Pulsed Doppler \\
\hline Energy & $100 \mathrm{~mJ}$ & TBD \\
\hline Pulse Repetition & $50 \mathrm{~Hz}$. & $50 \mathrm{~Hz}$. \\
\hline Afperture & $20 \cdot \mathrm{cm}$ & $20 \mathrm{~cm}$ \\
\hline Azimuth Scan & $90 \mathrm{deg} / 30 \mathrm{sec}$ & $90 \mathrm{deg} / 30 \mathrm{sec}$ \\
\hline Elevation & Manual Set & Manual Set \\
\hline Range Scan & $0-200 \mathrm{~m}$ in $0.25 \mathrm{sec}$ & $0-200 \mathrm{~m}$ in $0.25 \mathrm{sec}$ \\
\hline Passband & $200 \mathrm{kHz}$ to $10 \mathrm{MHz}$ & 1 to $50 \mathrm{MHz}$ \\
\hline
\end{tabular}

\section{VI-5.1. IIDAR Sensor}

The PLACEM Lidar [Emmitt, DiMarzio, and Doll] was designed and built for Pier IX Terminal Associates, of Newport News, VA, through a group effort between Northeastern University and Simpson Weather Associates. Simpson Weather Associates retains the intellectual right to PLACEM while Pier IX retains an interest in future copies of the system. The system consists of a LIDAR sensor and a computer data acquisition and control system.

The sensor specifications are as follows, and a photograph is shown in Figure 6:

- Pulse energy $=100 \mathrm{~mJ}$

- Wavelength $=10.6$ micrometers

- Pulse Length = 100 nanoseconds ( 15 meters of range resolution)

- Pulse repetition rate to $50 \mathrm{~Hz}$.

- Receiver aperture $=20 \mathrm{~cm}$., coaxial with tranśmitter

- Angular coverage = hemispherical, with a nominal 10-second/360-degree azimuth scan

- Interface: All equipment controlled by PC interface from a distance up to 100 meters.

- Power consumption less than 20 amps, single-phase, 120 V, $60 \mathrm{~Hz}$.

- Logistics: Carbon dioxide gas for laser and liquid nitrogen for detector.

- Weight = approximately $300 \mathrm{~kg}$.

- Dimensions: $180 \mathrm{~cm}$ high by $100 \mathrm{~cm}$ wide, by $80 \mathrm{~cm}$ deep.

A layout of the PLACEM system is shown in Figure 7. The transmitter laser, located in the bottom of the unit, is a carbon dioxide laser, with a pulse energy of more than 100 milliJoules and a pulse width of 


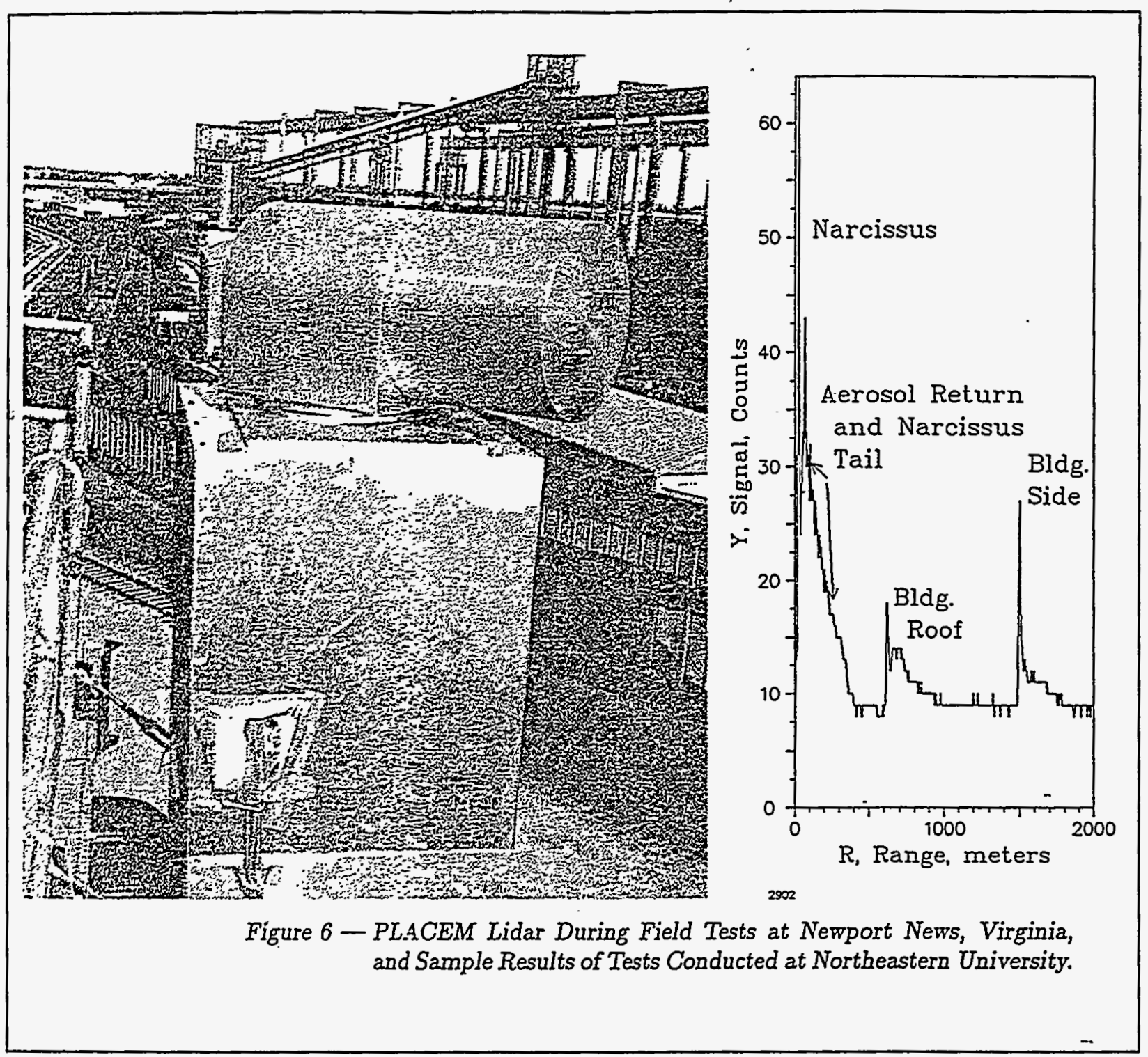

less than 100 nanoseconds. The pulse repetition frequency is controlled by computer with a maximum value of $50 \mathrm{~Hz}$. Light from the laser is directed to the scanner through a series of folding mirrors.

The scanner consists of two mirrors with a clear aperture of 20 centimeters, arranged so that they can scan a complete hemisphere in azimutb and elevation. These mirrors are controlled by a separate computer which generates the desired scan patiern.

Light returned from the target passes through the scanner into the telescope, which focuses it, via a folding mirror, onto a liquid-nitrogen-cooled, mercury cadmium telluride detector, with a frequency response of $100 \mathrm{MHz}$. The electrical signal from this detector is amplified and delivered to a digitizing card in the computer. This data may be displayed in raw form during collection. It is also passed to a SUN SparcStation for processing and display. 


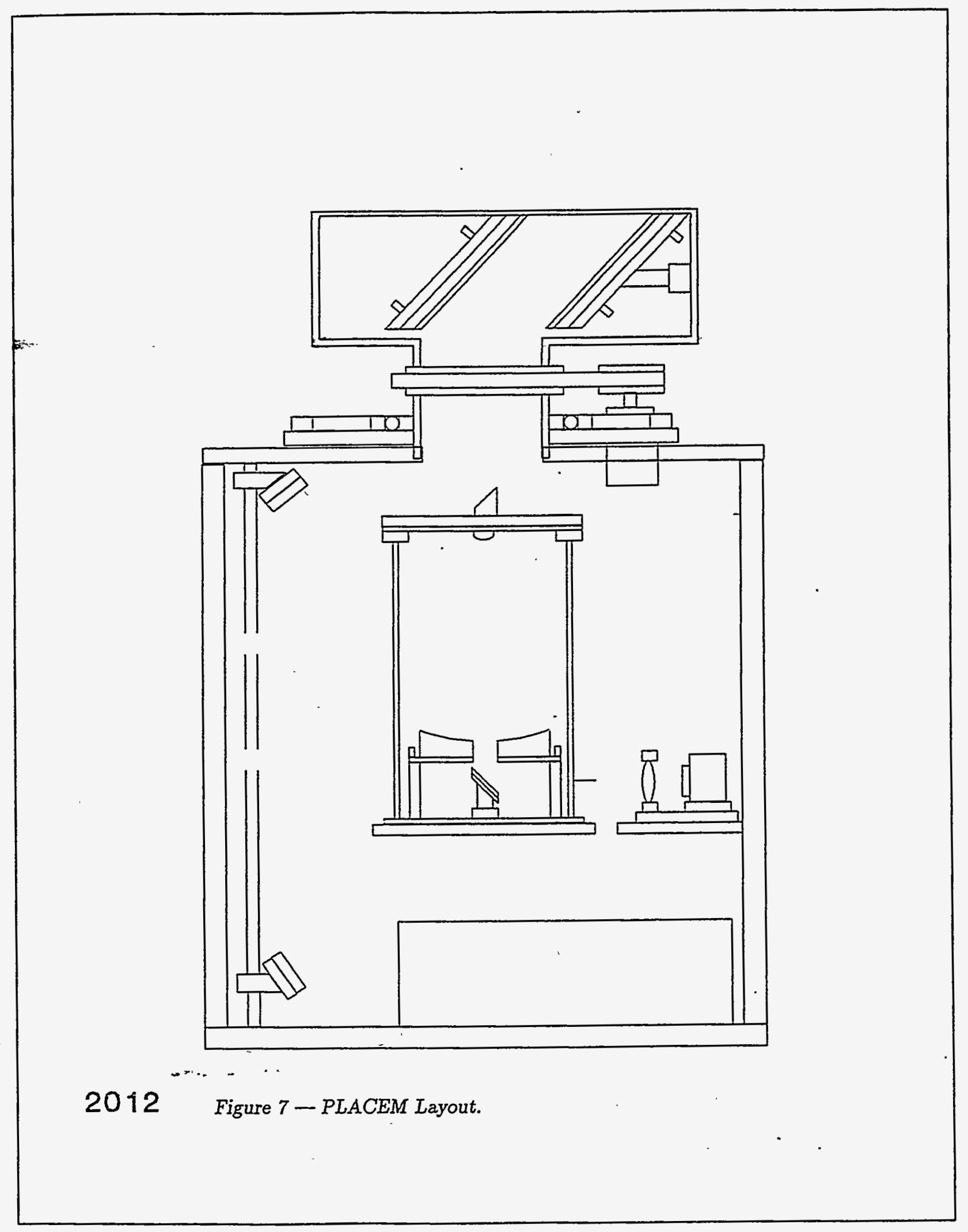

VI-12 
The computer system consists of a SUN Workstation, a 486 personal computer and the scanner control system, which includes a 386 personal computer. These three elements work together to produce all data acquisition, data processing and computer control. Figure 8 is a block diagram of the computer data acquisition and control system.

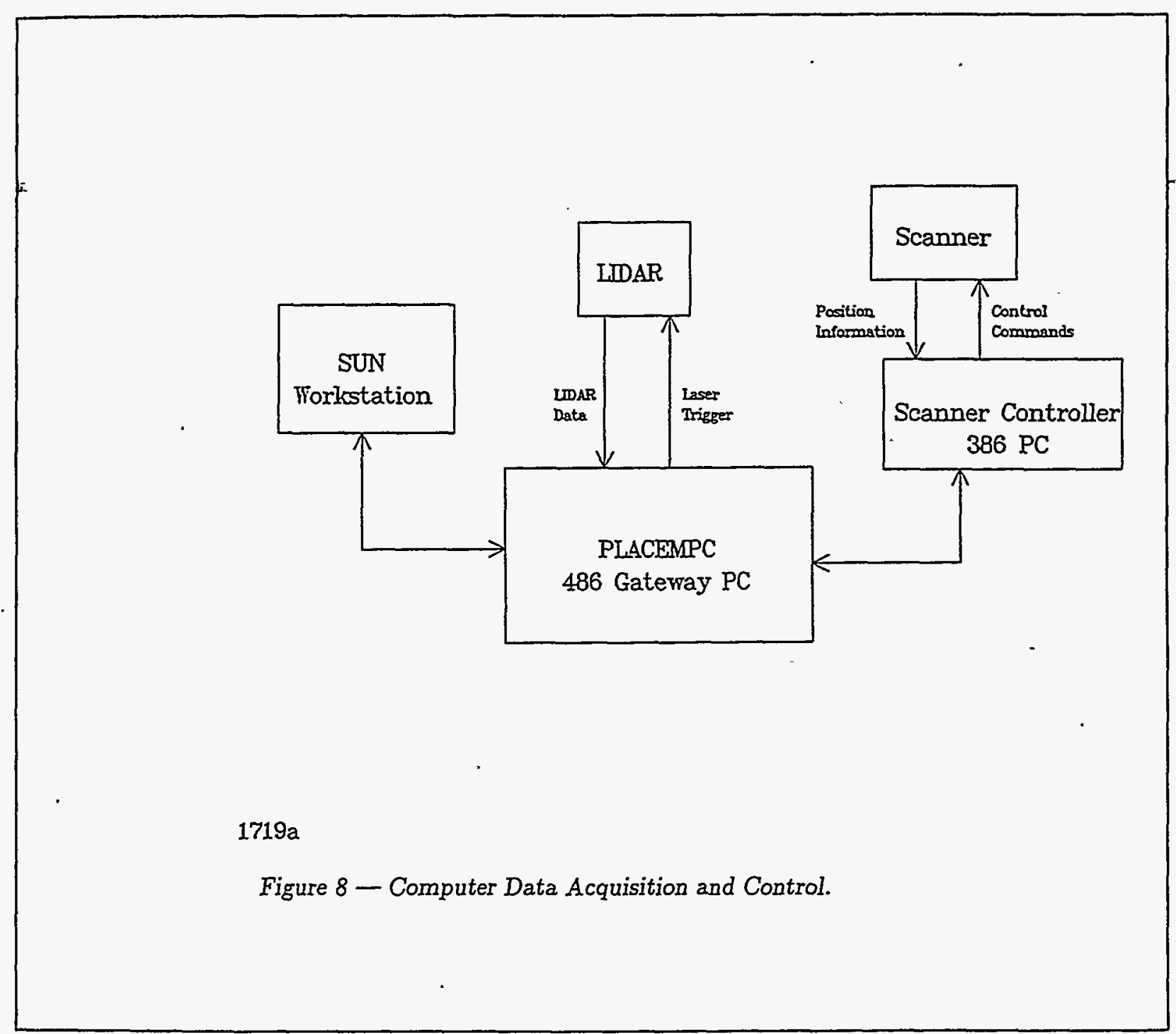

The SUN Workstation is configured to initiate all computer control and data acquisition. The SUN accepts the data collected by the 486 and processes the data. The data is displayed on an aerial map of the 


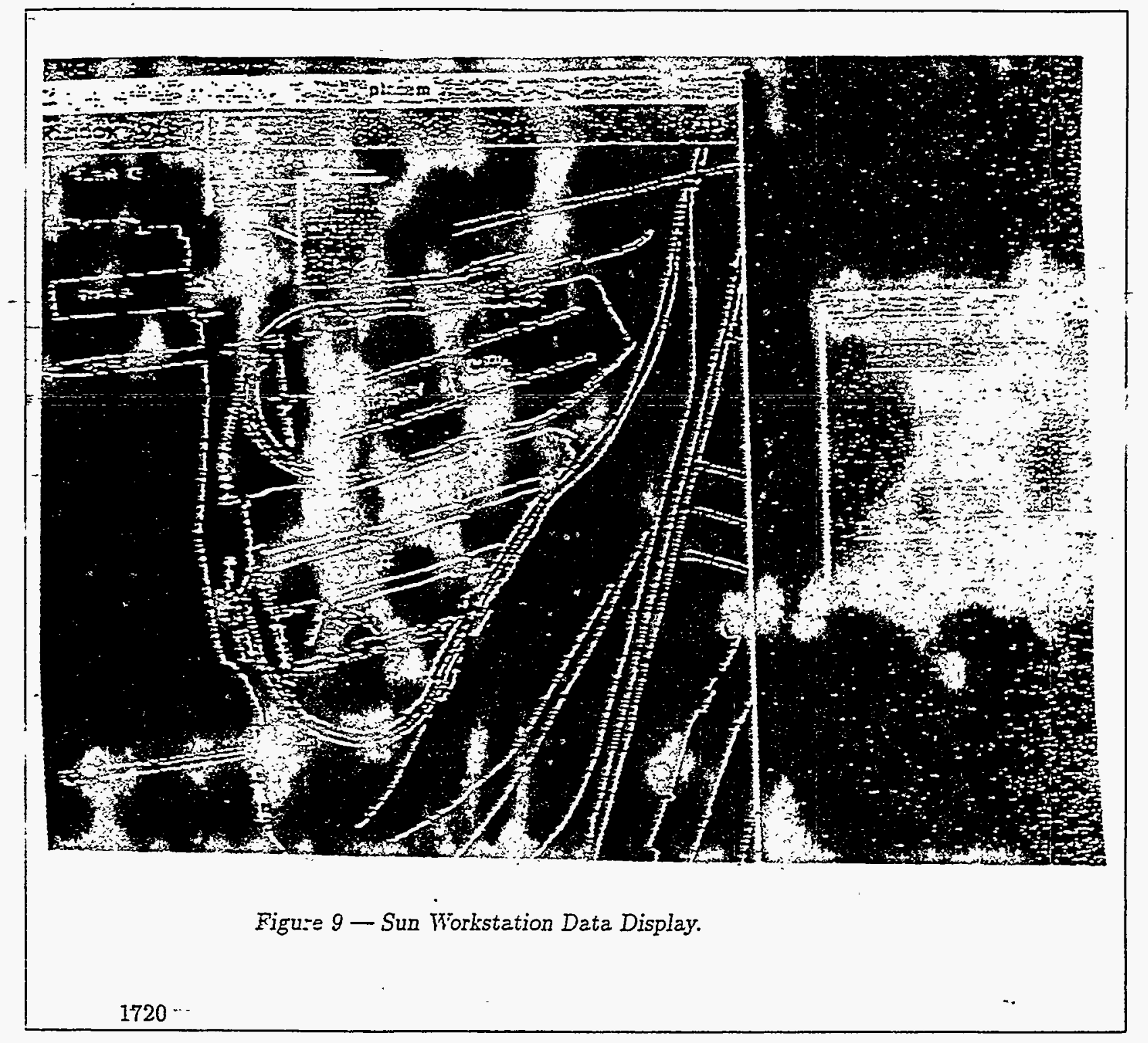

test sils informing the user of where fugitive emissions have been detected. Figure 9 shows the display as it appears on the SUN.

The 486 computer contains the data acouisition hardware, sends the t-igger signal to the laser system and sends the scinner ce-irol commands to.the 386 computer which contr: is the scanner. In software, a . trigge: signal is :reated which acts as a trigger fic: both the laser system and the data acquisition board. Upon zeceipt of ais trigger, the laser head transmits a laser pulse. At the same time, the data acquisition boaro proceeds to collect data. The data ècquisition board collects data at a rate of $25 \mathrm{MHz}$ and does so for a period of time that guarantees coverage of 1000 meters by the laser pulse. The collected data is sent to the $\mathrm{Si}$ i workstation and can also be saved to the disk. The 486 also delivers the scanner control commands to the $38 \hat{0}$ computer depending upon the scan pattern that has be established. It also acquires the position iniormation of the scanner from the 386 . 
Currently the display analysis software consists of three primary modules:

1) $P P I$ and RHI scan displays

2) Sector scan accumulation mode

3) Property line flux estimation.

The PPI/RHI scans are the standard sampling routines for mapping backscatter structures. These scan modes are used primarily during system checkout.

The accumulation mode is most frequently used since it builds an inventory of emission events for each 10-by-10 m grid over a prescribed period of time (e.g., I hour or 1 day).

At any time, one can quickly identify those sources associated with the most frequent and/or intense fugitive dust emission.

The code that computes a property line flux of particulates uses local wind observations $(5 \mathrm{sec})$ and multiple 360 degree scans to estimate the gradient of backscatter at large distances from the primary dust sources. A first order estimate of upwind/downwind mass concentrations can be derived from this sampling and analysis mode.

\section{VI-6. Recommendations.}

In order to determine the feasibility of LIDAR for making quantitative mass particulate measurements in a field environment, it is first necessary to determine if it is possible to make these measurements in a controlled laboratory environment and to look at scatter from individual particles. To do so requires developing an experimental setup capable of making backscatter measurements of known particle size distributions in a given volume.

Figure 10 shows the layout for an experimental setup for measuring particle backscatter from a known concentration of particles. The setup consist of three main parts: (1) illumination sources (lasers), (2) the particle test chamber, and (3) signal detection. A known size distribution of particles is suspended in the test chamber. This particle volume is illuminated with a single wavelength of focused laser energy. Upon interaction of the laser with the particle, the laser energy will be scattered in all directions, according to a pattern determined by the particle composition, size, relative to the laser wavelength, and the particle shape. Some laser energy will be backscattered towards the direction of the source. This energy will be focused on an appropriate detector, producing an electrical current proportional to the optical power. Focusing of the lasers and detectors limits the field-of-view and focal volume, which results in a reduction of the signal contributions from the beam stop after the test chamber. The resulting signal will be a measure of the total backscatter cross-section of the particles in the sample volume. However, it is possible for many different particle volumes, consisting of varied particle sizes, to produce the identical total backscatter cross-section. As a result, the experiment will be repeated at several different wavelengths. From the results from multiple wavelengths, it is hoped to be able to determine a mass concentration that would produce the backscatter cross-section which is unique to the particular combination of wavelengths. The HeNe laser can provide visible diffraction patterns resulting from interaction with individual particles.

Existing Mie scattering algorithms will be used to determine the expected backscatter cross-section from known particle sizes and distributions. Figure 11 shows the theoretical backscatter cross-section for coal particles of varying size, at three different wavelengths. These are based on previous research by the 


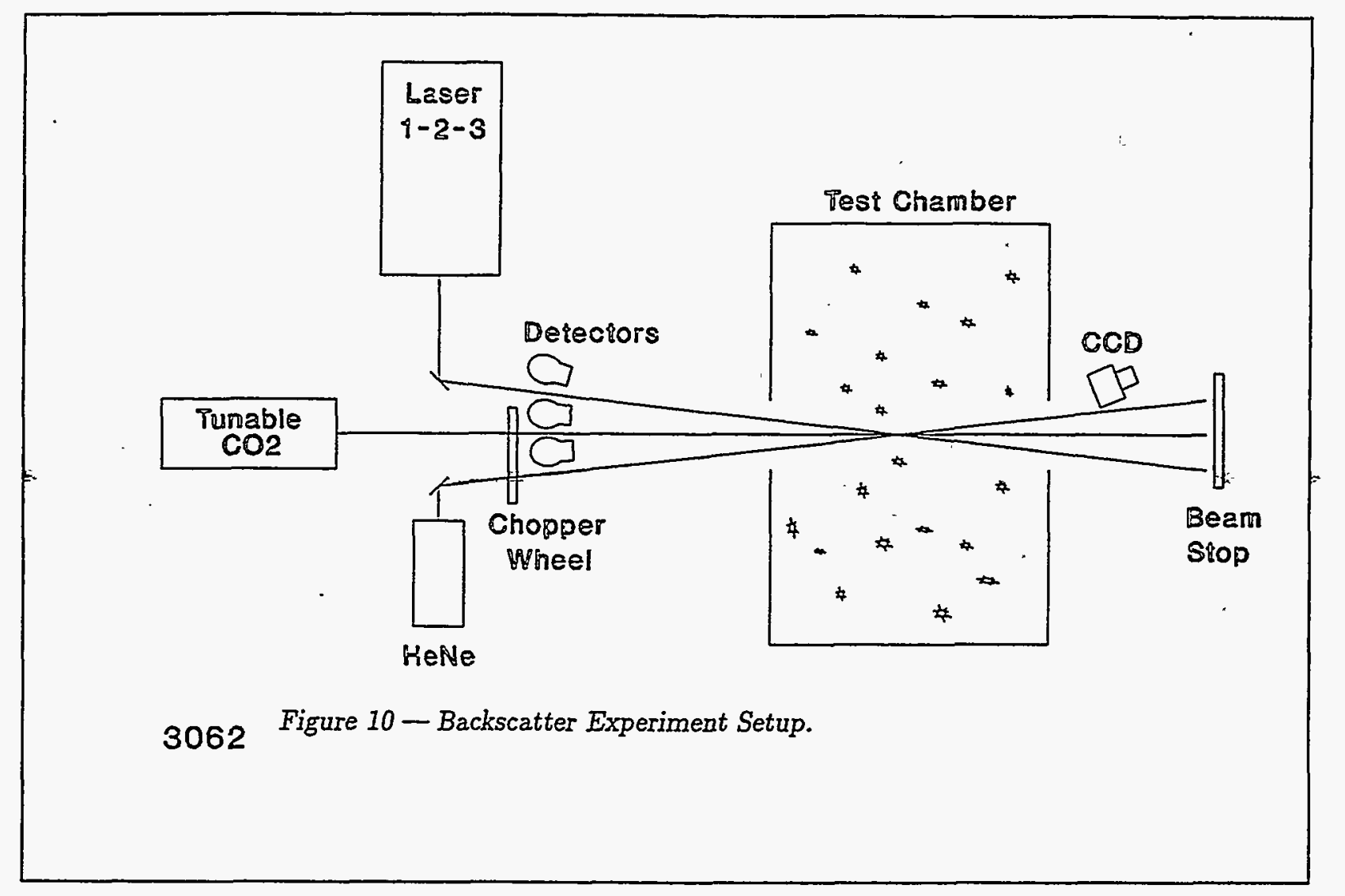

CER. Scattering from typical soil particles will probably be considerably stronger. The figure assumes a spherical particle. Collections of different particle shapes in random orientations can usually be modelled by smoothing out the variations in the Mie scattering curves for spherical particles. The existing algorithms can calculate the backscatter cross-section for any particle for which the complex index of refraction is known. Additionally, the algorithms can be modified to provide the backscatter cross-section for a distributed particle target. This information would then be incorporated into the experimental setup to assist in the concentration measurement. Measured backscatter cross-sections will be compared with the Mie scattering results to establish parametric fits which, would then be used to predict the particle distribution. Additionally, the algorithms can calculate the phase function for the scattered signal, providing a diffraction pattern for the forward direction. The diffraction pattern will vary according to the particle size. As a result, acquisition of the diffraction pattern, at red wavelengths with the CCD, will provide particle sizing information.

The desired experimental setup will be constructed with a combination of new equipment, to be purchased, and existing equipment currently available for this project. For the laser sources, the tunable $\mathrm{CO}_{2}$ will be purchased. This laser has the ability to be wavelength tuned to produce $\mathrm{CW}$ energy at a number of wavelengths between $9.2 \mu \mathrm{m}$ and $11 \mu \mathrm{m}$. The 1-2-3 is an existing, pulsed, Schwartz Electro-Optics laser, with separate optics for producing 1.06 $\mu \mathrm{m}, 2.09 \mu \mathrm{m}$ and $2.9 \mu \mathrm{m}$ laser energy. The HeNe laser is an existing $\mathrm{CW}$ laser operating at $633 \mathrm{~nm}$. The $\mathrm{CO}_{2}$ laser and the HeNe will pass through a chopper wheel, before reaching the test chamber, to produce a pulsed transmitter, as with the Laser 1-2-3.

The test chamber will consist of an enclosed box with openings at the front and back which allow the lasers to enter and exit the test volume. This is due to the fact that $\mathrm{CO}_{2}$ wavelengths do not pass through typical glass materials and using special windows (such as $\mathrm{ZnSe}$ ) adds unnecessary expense to the project. The size openings will be sufficiently small such that the sample volume will not decrease noticeably due to 


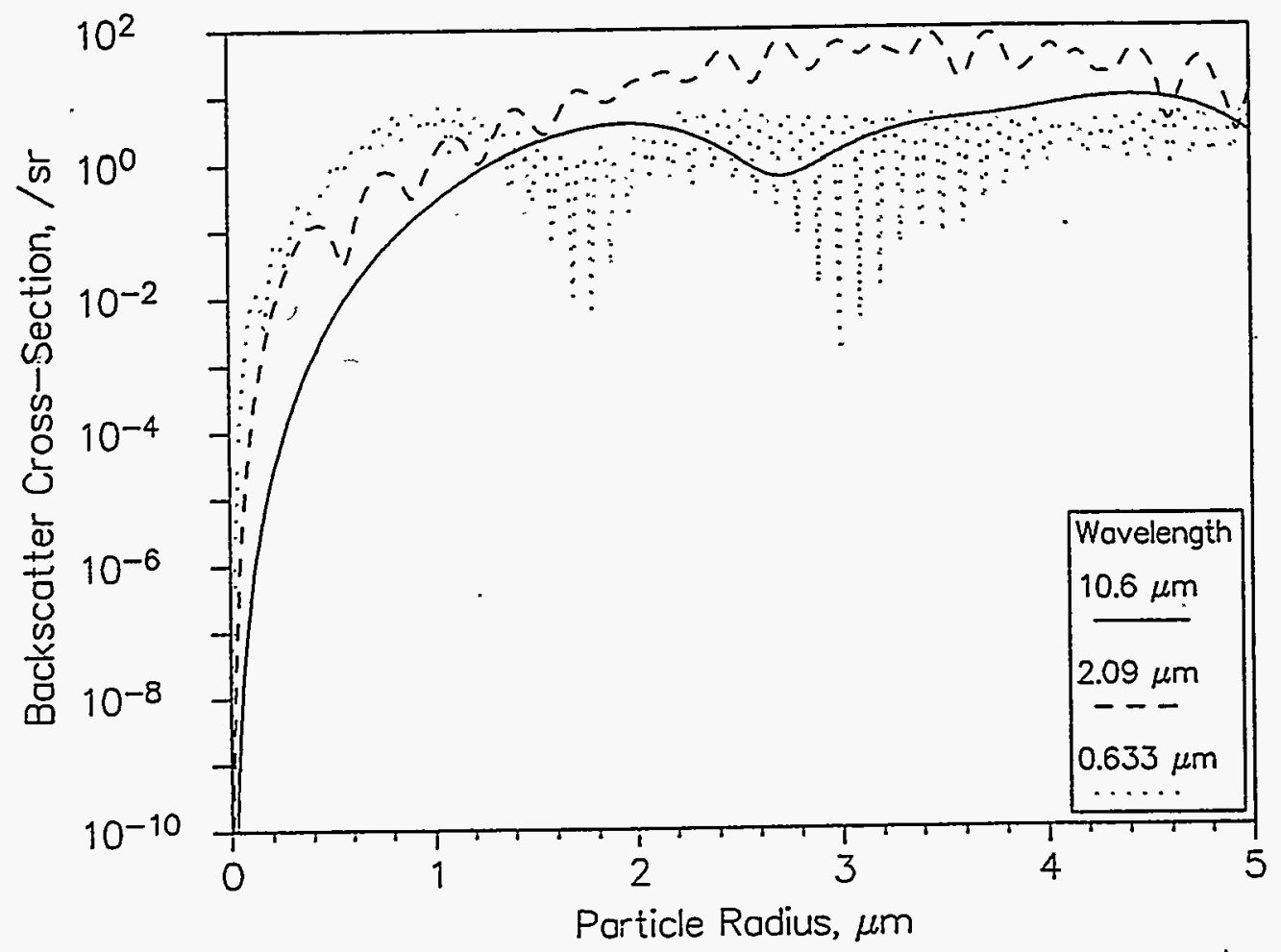

Figure 11 - Mie Scattering Results.

leakage through the openings. The particle sample will be deposited from the top of the chamber, allowing the sample to pass through the focused laser beam. A Real-time Aerosol Monitor (RAM) will be set up to monitor the dust concentration within the test chamber, providing $\mu g / \mathrm{m}^{3}$.

The backscattered signal will be measured with an appropriate detector. The $\mathrm{CO}_{2}$ wavelengths require a HgMnTe detector to provide the necessary sensitivity. The 1-3 $\mu \mathrm{m}$ wavelengths can be measured with InSb detectors. The HeNe can be detected with silicon photo-diodes. As mentioned previously, the diffraction pattern of the forward scattered signal can provide sizing information. For this reason, an existing $C C D$ camera, sensitive in the visible and near-IR range, will be positioned behind the test chamber. The CCD will be used to acquire 2-dimensional images of the diffracted forward signal. These images can be manipulated to provide the particle size.

One factor that can cause incorrect particle sizing is $\mathrm{H}_{2} \mathrm{O}$ bound to the particles. The backscatter cross-section of a particle having water content will be different than the same particle with no water 
content. This is due to the fact that the absorption spectrum of $\mathrm{H}_{2} \mathrm{O}$ varies as a function of wavelength. At certain wavelengths, $\mathrm{H}_{2} \mathrm{O}$ absorption will be low, thus making the $\mathrm{H}_{2} \mathrm{O}$ appear transparent to the laser, thus producing the backscatter cross-section of the particle itself. Figure 12 shows the extinction coefficient for $\mathrm{H}_{2} \mathrm{O}$ in the $200 \mathrm{~nm}$ to $200 \mu \mathrm{m}$ wavelength range [Hale and Querry]. As shown by the figure, $\mathrm{H}_{2} \mathrm{O}$ has distinct wavelength variations in the extinction coefficient and by carefully choosing the operating wavelengths, it may be possible to determine the sample water content as well as the particle size distribution in the sample.

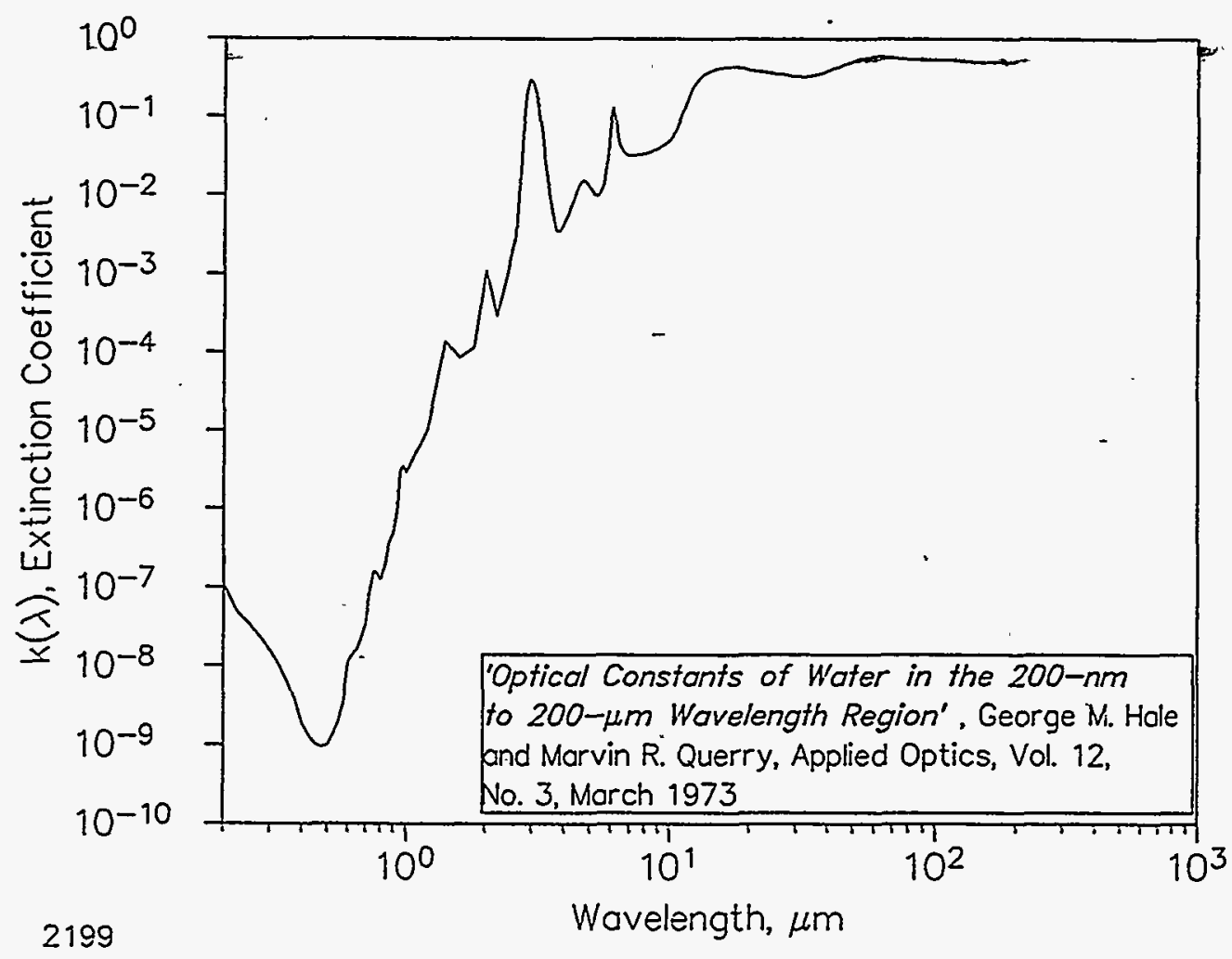

Figure 12 - Extinction Coefficient for $\mathrm{H}_{2} \mathrm{O}$. 


\section{VI-R. References}

Fluckiger, D. U., R. J. Keyes, and J. H. Shapiro, "Optical Autodyne Detection: Theory and Experiment," Applied Optics 26, No. 2, 15 Jan 1987. pp. 318-324.

DiMarzio, C. A., C. E. Harris, J. W. Bilbro, E. A. Weaver, D. C. Burnham, and J. N. Hallock, "Pulsed Laser Doppler Measurements of Wind Shear," Bulletin of American Meteorological Society,September 1979. pp. $1061-1066$.

Simon, Stuart A., Optical Amplification to Increase the Signal-to-Noise Ratio, MS Thesis in Electrical Engineering, MIT, Cambridge, MA. 1988.

Nordstrom, Robert J., and Lawrence J. Berg, "Coherent Laser Radar: Techniques and Applications," Lasers and Optronics 9, No. 6, June, 1990. Pp. 51-56.

Kane, Thomas J., Bingkun Zhou, and Robert L. Byer, "Potential for Coherent Doppler Wind Velocity LIDAR using Neodymium Lasers, Applied Optics 23, No. 15, Aug 1, 1984. 2477-2481.

Hale, George M., and Marvin R. Querry, "Optical Constants of Water in the 200-nm to 200- $\mu \mathrm{m}$ Wavelength Region." Applied Optics, Vol. 12, No. 3, March, 1973. pgs. 555-563.

Emmitt, G. D., Charles A. DiMarzio, and Roger Doll, "A Continuous Emission Monitoring and Modelling (CEM/M) System for Fugitive Particulate Emissions from Coal Handling Complexes," presented at 96th National Western Mining Conference, Denver, CO, March 1993. 